
DIRECT NUMERICAL SIMULATION OF PIPE FLOW USING A SOLENOIDAL SPECTRAL METHOD

A THESIS SUBMITTED TO

THE GRADUATE SCHOOL OF NATURAL AND APPLIED SCIENCES

$\mathrm{OF}$

MIDDLE EAST TECHNICAL UNIVERSITY

BY

OZAN TUĞLUK

IN PARTIAL FULFILLMENT OF THE REQUIREMENTS

FOR

THE DEGREE OF DOCTOR OF PHILOSOPHY

IN

ENGINEERING SCIENCES

APRIL 2012 
Approval of the thesis:

\section{DIRECT NUMERICAL SIMULATION OF PIPE FLOW USING A SOLENOIDAL SPECTRAL METHOD}

submitted by OZAN TUĞLUK in partial fulfillment of the requirements for the degree of Doctor of Philosophy in Engineering Sciences Department, Middle East Technical University by,

Prof. Dr. Canan Özgen

Dean, Graduate School of Natural and Applied Sciences

Prof. Dr. Turgut Tokdemir

Head of Department, Engineering Sciences

Assoc. Prof. Dr. Hakan I. Tarman

Supervisor, Department of Engineering Sciences, METU

\section{Examining Committee Members:}

Prof. Dr. Münevver Tezer-Sezgin

Department of Mathematics, METU

Assoc. Prof. Dr. Hakan I. Tarman

Department of Engineering Sciences, METU

Assoc. Prof. Dr. Utku Kanoğlu

Department of Engineering Sciences, METU

Assist. Prof. Dr. Cüneyt Sert

Department of Mechanical Engineering, METU

Assist. Prof. Dr. Nilay Sezer Uzol

Department of Mechanical Engineering, TOBB ETU

Date: 
I hereby declare that all information in this document has been obtained and presented in accordance with academic rules and ethical conduct. I also declare that, as required by these rules and conduct, I have fully cited and referenced all material and results that are not original to this work.

Name, Last Name: OZAN TUĞLUK

Signature 


\begin{abstract}
DIRECT NUMERICAL SIMULATION OF PIPE FLOW USING A SOLENOIDAL SPECTRAL METHOD

\author{
Tuğluk, Ozan \\ Ph.D., Department of Engineering Sciences \\ Supervisor : Assoc. Prof. Dr. Hakan I. Tarman
}

April 2012, 99 pages

In this study, which is numerical in nature, direct numerical simulation (DNS) of the pipe flow is performed. For the DNS a solenoidal spectral method is employed, this involves the expansion of the velocity using divergence free functions which also satisfy the prescribed boundary conditions, and a subsequent projection of the N-S equations onto the corresponding dual space. The solenoidal functions are formulated in Legendre polynomial space, which results in more favorable forms for the inner product integrals arising from the Petrov-Galerkin scheme employed. The developed numerical scheme is also used to investigate the effects of spanwise oscillations and phase randomization on turbulence statistics, and drag, in turbulent incompressible pipe flow for low to moderate Reynolds numbers (i.e. Re $\sim 5000)$ ).

Keywords: pipe flow, drag reduction, transition to turbulence, turbulence, direct 
numerical simulation, turbulence control, transition control, transient growth, wallbounded flows, spanwise oscillations, phase randomization, solenoidal basis, pseudospectral methods 


\title{
ÖZ
}

\section{SOLENOIDAL SPEKTRAL YÖNTEM KULLANILARAK BORU AKIŞININ DOĞRUDAN SAYISAL BENZETIMI}

\author{
Tuğluk, Ozan
}

Doktora, Mühendislik Bilimleri Bölümü

Tez Yöneticisi : Doç. Dr. Hakan I. Tarman

Nisan 2012, 99 sayfa

$\mathrm{Bu}$ çalışmada, sıkıştırılamaz boru akışında açısal salınımların ve faz randomizasyonunun, düşük-orta Reynolds sayıları (Re 5000)) için türbülans istatistikleri ve sürüklenme üzerindeki etkileri araştırılmıştır. Bu çalışmada, sayısal nitelikli olup, boru akışının direkt sayısal simülasyonu (DNS) yapılmıştır. DNS için solenoidal spektral yöntemi kullanılmış olup, bu yöntem öngörülen sınır şartlarını karşılayan solenoidal bazlarla hız vektörünün açılımını ve sonrasında, ilgili ikili vektör uzayı üzerine NS denklemlerinın projeksiyonunu içerir. Solenoidal baz fonksiyonları Legendre polinom uzayında formüle edilmiş olup, kullanılan Petrov Galerkin şemasından kaynaklanan iş çarpımlar için daha elverişli formlar sunmaktadır.

Anahtar Kelimeler: boru akışı, türbülansa geçiş, türbülans, direkt sayısal simülasyon, türbülans kontrolü, geçiş kontrolü, geçici büyüme, duvara sınırlı akış, açısal salınımlar, 
faz rastlaştırma, solenoidal bazlar, pseudospectral yöntemler 
To Ali and Şadan (in alphabetical order) ... 


\section{ACKNOWLEDGMENTS}

I am grateful to my parents, Ali and Şadan (in alphabetical order), for their continual support (emotional and financial), encouragement, love, and friendship. I would also like thank my brother, Okan, and my grandmother Şükran who initiated my scientific eduation via her kitchen experiments. Thanks also go the other members of the family as well.

I would also wish to express my gratitude to my supervisor Dr. Hakan Tarman and the thesis examining comitee members for their criticism, guidance, motivation throughout the research.

I would like to thank Özlem for existing against all odds.

I want to express my gratitude for the peoples of baraka, and the household of DC59, for their continual friendship and more through space and time. I would also like to thank Dr. Güllü, Dr. Beyaz, Dr. Sarığlu, and Dr. Zheltukhin for the thought provoking discussions we have had over lunch, which have greatly aided in my determination to continue with my work.

My deepest thanks to Emre and Oya Sezginer for their support and encouragement, and to Ozan Saygın Caner, Banu Camgözoğlu and Cengiz Acartürk for supplying me with model M keyboards.

I am deeply deeply indebted to Nilgün Öner for her presence after my defense, as well her logistical support afterwards, I am also indebted to Feride Mutlu, Burcu, Çă̆rı, Gülistan, Tuğba and Zelha for their pleasant suprise.

The company of the frequenters, and the staff of beşeri kantini, was also invaluable.

This study was supported in part by the Scientific and Technical Research Council of Turkey (TÜBİTAK) through research project 109M435. I would also like to thank the organizing comitee of Turbulent Mixing and Beyond 2009, and ICTP for their support 
during the conference, and the Basque Center for Applied Mathematics (BCAM) for their support during OPTPDE 2012.

Last, but certainly not the least, the use of Debian GNU/Linux and a collection of free software, which continues exist thanks to the members of the free software community, which were indespensible for the presented work, is greatly acknowledged. 


\section{TABLE OF CONTENTS}

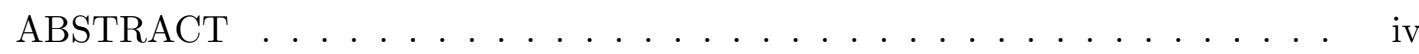

$\ddot{\mathrm{O} Z}$

ACKNOWLEDGMENTS . . . . . . . . . . . ....... ix

TABLE OF CONTENTS $\ldots \ldots \ldots \ldots \ldots$ xi

LIST OF FIGURES . . . . . . . . . . . . . . . . . . . . . . . . . . . xiii

LIST OF TABLES . . . . . . . . . . . . . . . . . . . . . . . . . xvi

LIST OF ABBREVIATIONS . . . . . . . . . . . . . . . . . . . . . . . xvii

\section{CHAPTERS}

$1 \quad$ INTRODUCTION $\ldots \ldots \ldots \ldots \ldots \ldots$

$1.1 \quad$ Turbulence $\ldots \ldots \ldots \ldots \ldots \ldots$

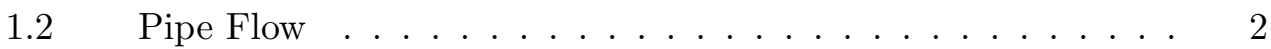

1.3 Transition to Turbulence in Pipe Flow . . . . . . . . . . . 3

$1.4 \quad$ Turbulent Drag Reduction $\ldots \ldots \ldots \ldots$

1.4.1 Drag Reduction via Spanwise Wall Oscillations . . . 7

1.4.2 Drag Reduction via Phase Randomization . . . . . 7

1.4.3 Other Drag Reduction Methods . . . . . . . . . . . 8

$1.5 \quad$ Solenoidal Basis Functions $\ldots \ldots \ldots$

$1.6 \quad$ What is new in this study $\ldots \ldots \ldots \ldots$

$1.7 \quad$ Outline . . . . . . . . . . . . . . . . . . . . . . . . . . 11

$2 \quad$ PIPE FLOW $\ldots \ldots \ldots \ldots \ldots \ldots$

$2.1 \quad$ Governing Equations $\ldots \ldots \ldots \ldots \ldots$

$2.1 .1 \quad$ Non-dimensionalization . . . . . . . . . . . . . . 13

$2.1 .2 \quad$ Laminar Flow . . . . . . . . . . . . . . . . . 17 
$3 \quad$ THE NUMERICAL METHOD $\ldots \ldots \ldots \ldots$

$3.1 \quad$ Basis Functions $\ldots \ldots \ldots \ldots$

$3.1 .1 \quad$ Construction of the Bases $\ldots \ldots \ldots \ldots$

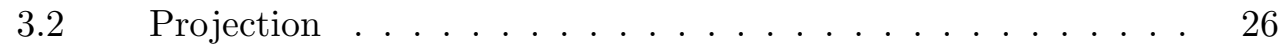

$3.3 \quad$ Time Solvers . . . . . . . . . . . . . . . . . . . . . . . . 27

$3.4 \quad$ The Computer Code $\ldots \ldots \ldots \ldots$

$4 \quad$ CODE VALIDATION $\ldots \ldots \ldots \ldots$

$4.1 \quad$ Laminar Test Case and Linear Stability . . . . . . . . . . . . . 33

$4.2 \quad$ Transient Growth of Perturbations . . . . . . . . . . . 35

$4.3 \quad$ Benchmark cases for turbulent pipe flow at low Reynolds number 43

$4.3 .1 \quad$ Constant Pressure Gradient Case . . . . . . . . . . . 43

$4.3 .2 \quad$ Constant mass flux . . . . . . . . . . . . 50

$4.3 .3 \quad$ Energy conserving property . . . . . . . . . . 52

5 DRAG REDUCTION STRATEGIES . . . . . . . . . . . . . . . . 54

$5.1 \quad$ Drag Reduction via Spanwise Oscillations . . . . . . . . . . . 54

$5.1 .1 \quad$ Constant Pressure Gradient Case . . . . . . . . . 55

$5.1 .2 \quad$ Constant Mass Flux Case $\ldots \ldots \ldots \ldots$

$5.2 \quad$ Phase Randomization . . . . . . . . . . . . . . . . . . 64

$5.2 .1 \quad$ Constant Pressure Gradient Case . . . . . . . . . 66

$5.2 .2 \quad$ Constant Mass Flux Case $\ldots \ldots \ldots$. . . . . . 68

$5.2 .3 \quad$ Drag Reduction Summary $\ldots \ldots \ldots$

$6 \quad$ CONCLUSION $\ldots \ldots \ldots \ldots \ldots$

$6.1 \quad$ Future Work . . . . . . . . . . . . . . . . . . . . . . . 82

REFERENCES . . . . . . . . . . . . . . . . . . . . . . . 84

\section{APPENDICES}

A Solenoidal Basis Functions . . . . . . . . . . . . . . . . . . . . . . . 91

B Constant Mass Flux Formulation . . . . . . . . . . . . . . . . . . . . . 93

CURRICULUM VITAE . . . . . . . . . . . . . . . . . . . . . . . 96 


\section{LIST OF FIGURES}

\section{FIGURES}

Figure 2.1 Pipe flow geometry $\ldots \ldots \ldots$

Figure 3.1 Clustered vs Non-Clustered Grids . . . . . . . . . . . . . . . . . . . 20

Figure 3.2 Mode $(0,1,0)$ radial contours $\ldots \ldots \ldots \ldots$. . . . . . . . . 27

Figure 3.3 Mode $(0,1,0)$ azimuthal contours . . . . . . . . . . . . . . . . 28

Figure $3.4 \quad$ Flowchart constant pressure . . . . . . . . . . . . . . . . . . . . . . . . 31

Figure $3.5 \quad$ Flowchart constant mass flux $\ldots \ldots \ldots$. . . . . . . . . . . . . 32

Figure 4.1 Stream function, $1=1, \mathrm{n}=1 \ldots \ldots \ldots \ldots$

Figure 4.2 Vector field, $\mathrm{l}=1, \mathrm{n}=1 \ldots \ldots \ldots \ldots$

Figure 4.3 Stream function, $1=0, n=1 \ldots \ldots \ldots$. . . . . . . . . . . . 37

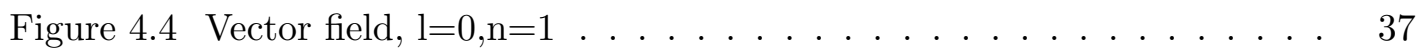

Figure 4.5 Stream function, $1=1, n=1 \ldots \ldots \ldots \ldots$

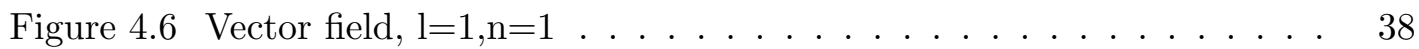

Figure 4.7 Evolution of $2 \mathrm{D}$ pertutbations $\ldots \ldots \ldots \ldots$

Figure 4.8 Streak formation $\ldots \ldots \ldots \ldots$

Figure 4.9 Decay od 2D perturbation energy $\ldots \ldots \ldots \ldots$. . . . . . . . . . . . 42

Figure 4.10 Mean axial velocity in the presence of oscillations . . . . . . . . . . 42

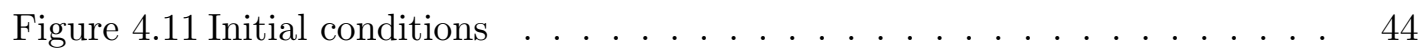

Figure 4.12 Semi-logarithmic plot of mean axial velocity . . . . . . . . . . . . . 47

Figure 4.13 Mean axial velocity scaled by mean centerline velocity . . . . . . . 47

Figure 4.14 RMS velocity fluctuations of $\mathrm{u} \ldots \ldots \ldots \ldots$. . . . . . . . . 48

Figure 4.15 RMS velocity fluctuations of $\mathrm{v} \ldots \ldots \ldots$. . . . . . . . . . 48 
Figure 4.16 RMS velocity fluctuations of $\mathrm{w} \ldots \ldots$

Figure 4.17 RMS velocity fluctuations $\ldots \ldots$. . . . . . . . . . . . . . . 49

Figure 4.18 Mean Reynolds stress . . . . . . . . . . . . . . . . . . . . . . 50

Figure 4.19 Mean streamwise velocity profiles . . . . . . . . . . . . . . . . . . 51

Figure 4.20 Mean streamwise velocity profiles $\ldots \ldots$. . . . . . . . . . . 51

Figure 4.21 Mean Reynolds stress $\ldots \ldots \ldots$. . . . . . . . . . . . . . . . . . 52

Figure 5.1 Time evolution of skin friction coefficient $\ldots \ldots \ldots$. . . . . . . 56

Figure 5.2 Mean streamwise velocity profiles $\ldots \ldots \ldots \ldots$. . . . . . . . 57

Figure 5.3 Mean Reynolds stress controlled and uncontrolled cases . . . . . . . 57

Figure 5.4 Mean urms for controlled and uncontrolled cases $\ldots \ldots$. . . . . . 58

Figure 5.5 Mean streamwise velocity profiles $\ldots \ldots$. . . . . . . . . . . 60

Figure 5.6 Mean streamwise velocity profiles $\ldots \ldots \ldots$. . . . . . . . . . 61

Figure 5.7 Mean Reynolds stress $\ldots \ldots \ldots$. . . . . . . . . . . . . . . . . 61

Figure 5.8 Urms for controlled and uncontrolled cases $\ldots \ldots \ldots$. . . . . . . . . 62

Figure 5.9 Vrms for controlled and uncontrolled cases . . . . . . . . . . . . 62

Figure 5.10 Wrms for controlled and uncontrolled cases . . . . . . . . . . . . 63

Figure 5.11 Pressure gradient $\ldots \ldots \ldots$. . . . . . . . . . . . . . . . 63

Figure 5.12 Mean Reynolds stress phase randomization controlled and uncontrolled cases . . . . . . . . . . . . . . . . . . . . 67

Figure 5.13 Mean urms for controlled and uncontrolled cases . . . . . . . . 67

Figure 5.14 Mean Reynolds stress $\ldots \ldots \ldots$. . . . . . . . . . . . . . . . 69

Figure 5.15 Urms for controlled and uncontrolled cases . . . . . . . . . . . . . 69

Figure 5.16 Stream lines at $\mathrm{t}=800$ constant mass flux, controlled and uncontrolled cases . . . . . . . . . . . . . . . . . . . . . . . 71

Figure 5.17 RMS radial velocity, time average, constant mass flux, controlled and uncontrolled cases. . . . . . . . . . . . . . . . . . . 74

Figure 5.18 RMS azimuthal velocity, time average, constant mass flux, controlled and uncontrolled cases. . . . . . . . . . . . . . . . . . 75 
Figure 5.19 RMS r-vorticity, time average, constant mass flux, controlled and uncontrolled cases. . . . . . . . . . . . . . . . . . . . 76

Figure 5.20 RMS $\theta$-vorticity , time average, constant mass flux controlled and uncontrolled cases. . . . . . . . . . . . . . . . . . . 77

Figure $5.21 \mathrm{RMS}$ z-vorticity,time average, constant mass flux, controlled and uncontrolled cases. . . . . . . . . . . . . . . . . . . . . . 78

Figure 5.22 Average rms r vorticity, wall region, constant mass flux, controlled 


\section{LIST OF TABLES}

\section{TABLES}

Table 4.1 Leading eigenvalue $R e_{c l}=9600 \ldots \ldots \ldots \ldots$

Table 4.2 Leading eigenvalue $R e_{c l}=3000 \ldots \ldots \ldots \ldots$

Table 4.3 Turbulence statistics for pipe flow in the cases of (1) constant pressure gradient, (2) constant mass flux. . . . . . . . . . . . . . . 46

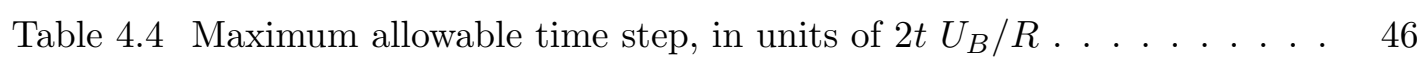

Table 4.5 Growth of kinetic energy as $R e_{\tau} \rightarrow \infty \quad \ldots \ldots \ldots$

Table 5.1 Comparison of turbulence statistics for controlled and uncontrolled flow, for fixed oscillation frequency $\Omega=6 \pi \ldots \ldots \ldots$. . . . . . . 56

Table 5.2 Comparison of turbulence statistics for controlled and uncontrolled flow, for fixed oscillation amplitude $A=10 \ldots \ldots \ldots$. . . . . . 58

Table 5.3 Comparison of turbulence statistics for controlled and uncontrolled flow in the case of constant mass flux. $G_{P}$ denotes the mean pressure gradient required for corresponding laminar flow. . . . . . . . . . . . . 59

Table 5.4 Effect of phase randomization for constant axial pressure gradient . $\quad 66$

Table 5.5 Comparison of turbulence statistics for controlled and uncontrolled flow, for fixed oscillation frequency $\Omega=6 \pi$ and phase randomization . . . 66

Table 5.6 Effects of phase randomization, constant mass flux $\ldots \ldots$. . . . . 68

Table 5.7 Turbulence statistics for controlled and uncontrolled flow, constant mass flux. 


\section{LIST OF ABBREVIATIONS}

$(\cdot)^{*} \quad$ : Normalized $(\cdot)$ in friction units

$(\cdot)^{+} \quad$ : Normalized $(\cdot)$ in wall units

$(\cdot)_{B} \quad$ : Normalized $(\cdot)$ in bulk units

2D : Three Dimensional

3D : Three Dimensional

EEF : Empirical Eigenfunction

EOF : Empirical Orthogonal Function

HPF : Hagen-Poiseuille Flow

KL : Karhunen-Loève

NS : Navier Stokes

ODE : Ordinary Differential Equation

PDE : Partial Differential Equation

POD : Proper Orthogonal Decomposition

UPO : Unstable Periodic Orbit

TW : Travelling Wave 


\section{CHAPTER 1}

\section{INTRODUCTION}

I am an old man now, and when I die and go to Heaven there are two matters on which I hope enlightenment. One is quantum electrodynamics and the other is turbulence of fluids. About the former, I am rather optimistic.

Sir Horace Lamb

\subsection{Turbulence}

Turbulence is a complex flow phenomenon, as a matter of fact most flows are turbulent in nature. One can cite the ocean currents, jet streams in the atmosphere, the surface of the sun, boundary layers and wakes created by aircraft, flow around an automobile, flow in canals and rivers, flow in oil and gas pipelines, the gaseous nebulae [1, as examples. In fact it would not be inaccurate to state that we, as human beings, spend our lives immersed in flow of turbulent fluids. Turbulence is, by its nature, a nonlinear, diffusive, three dimensional, unsteady, rotational and multiscale phenomenon which involves three dimensional vorticity fluctuations. Hence many simplifying assumptions of theoretical fluid mechanics are not valid for turbulent flow, so theoretical advances in turbulence have been rather limited. It is widely accepted that the venerable Navier Stokes equations adequately describe turbulence [2, 3] by admitting turbulent solutions. However having such an admittedly complete mathematical description does not alleviate the problems encountered in theoretical approach to turbulence, as existence and smoothness of Navier-Stokes solutions in three space dimensions itself 
is one of the seven Millennium Prize Problems set by the Clay Institute. Owing to these difficulties, it would not be inaccurate to state that turbulence research has been a basin of attraction, borrowing the term from dynamical systems, for studies which are numerical in nature.

Returning to the nature of turbulence, the main implication of turbulent flow is that the drag in a wall-bounded turbulent flow is always higher than the drag in the corresponding laminar flow. The difference in the drag force can be orders of magnitude depending on flow parameters such as viscosity and mean velocity. From an engineering perspective this is certainly an undesirable characteristic of turbulent flows. In addition to requiring more energy to drive, turbulence enhances mixing and heat transfer. Enhancement of mixing and heat transfer are often desirable but depending on the application one might want to minimize them as well.

Turbulence arises from instabilities in the flow, whether these are global or local instabilities is a matter which is debated. As the nature of the instabilities is also dependent on the flow geometry, next section gives the background for pipe flow, which is considered in this study as a test case, and in the following section transition to turbulence is discussed.

As a side note, we would like to stress that by the word turbulence we mean hydrodynamic or Navier-Stokes turbulence [1, 4, 5. This has been stressed as the word turbulence has gained recognition in dynamical systems community in a broader sense to describe all spatio-temporally complex phenomena [6, 7, 8].

\subsection{Pipe Flow}

The geometry of choice in this study is pipe flow, which is essentially flow in a cylindrical pipe with circular cross section. The reason why this geometry is chosen is twofold. From an application point of view, the industrial relevance and importance of pipe flow is evident. Pipes with circular cross section are very widely utilized in various industries and applications, such as oil and gas pipe lines, water and sewage systems, fuel injection systems in space, air, sea and land vehicles, cooling systems etc. Also pipe flow experiments are easier and cheaper to conduct than other wall 
bounded flow geometries [9]. One interesting property of pipe flow, similar to Couette flow, is that even though the flow in a pipe is linearly stable (see section 1.3), it certainly can and does become turbulent. Furthermore the Navier-Stokes equations have coordinate singularities when formulated in cylindrical coordinates. Hence, pipe flow, is one of the more difficult cases of wall-bounded flows, even though the geometry is relatively simple, mainly due to the coordinate singularities.

Historically the pioneering work on pipe flow was performed by Jean Louis Marie Poiseuille [10], a physician and physiologist, who was mainly interested in the flow of blood within the veins, Gotthilf Hagen [11, a physicist and hydraulic engineer, and Osborne Reynolds [12]. The first studies on the subject were experimental, as the theoretical approach was, and is, much more difficult (see Sec. 1.1).

\subsection{Transition to Turbulence in Pipe Flow}

Stability of pipe flow has been studied for over a century, ever since the well known experiments by Reynolds [12]. In his experiments Reynolds observed how direct flow in a pipe became sinous. This phenomena, which is now called transition to turbulence, has been subject to many diverse numerical, theoretical and experimental studies. It is widely accepted that, the sole flow parameter for this phenomena is the Reynolds number. Even though the pipe flow is geometrically very simple, and experiments are relatively easy to conduct in this setting, the theoretical studies were limited, and the numerical approach was more widely used. One of the main peculiarities of pipe flow is that the flow is linearly stable [13] at even very high Reynolds numbers, upto $R e \sim 10^{7}$, as it was shown in [13]), causing a decay in small perturbations. With linear stability it is meant that when the Navier-Stokes equations are linearized around the laminar solution, the eigenvalues corresponding to the linearized system all have negative real parts (for a detailed discussion consult [13]). The computations in this study also verify that the associated eigenvalues have negative reals parts as expected, this has been used as an initial validation case in section 4.1. The presence of linear stability means, for transition in pipe flow to occur, both the flow speed and the perturbation amplitude must be sufficiently high [14]. From a mathematical standpoint, this means that the laminar solution does not offer a bifurcation point 
at finite values of Reynolds number [15] and for infinitesimal values of disturbances. It has been known for over a century, that the Reynolds number $(R e=U D / \nu$ as defined by Reynolds himself, where $U$ is the mean axial flow velocity), is the single dimensionless flow parameter which governs the transition to turbulence in pipe flow [12. When Osborne Reynolds first observed transition to turbulence in pipe flow, he wrote: "All the same I felt a certain amount of uncertainty in assuming the first cause of instability to be general. This uncertainty was the result of various considerations, but particularly from my having observed that eddies apparently come on in very different ways, according to a very definite circumstance of motion ..." [12]. In his aforementioned article, Reynolds also observes that there is no global critical value for transition to turbulence, rather the transition is strongly linked to the perturbations applied to the flow.

Recent studies also suggest that pipe flow undergoes transition in the absence of mediation by a linear instability of the laminar profile, and it does not seem possible to find a spatially and temporally simple intermediate state (like the famous Rayleigh-Benard rolls for example) between turbulent and laminar regimes [14. These observations, taken from both numerical and theoretical studies, led most, if not all, of the turbulence research community to consider the turbulent state represent a chaotic saddle in the state space [14]. To further complicate the problem, it is still debated whether the transition is a global or local phenomena [16]. Another complication is that it has been shown, both numerically and experimentally, that turbulent states may decay and degenerate into laminar flow without clear precursors [16, 17, 18], if the observation time is sufficiently long [14].

As Kerswell clearly states in [15], the main issue with transition to turbulence in general, and transition in pipe flow in particular, is not the governing equations or their solution. We know that the Navier-Stokes equations are not lacking in these transition regimes [8], further their solution can be obtained by a myriad of numerical methods. The problem lies in the analyzing and rationalization of the obtained solutions to this set of nonlinear equations. In simulations of Navier-Stokes equations, one generally obtains a system of ODE's with quadratic nonlinearity. The degree of freedom routinely ranges from $\mathcal{O}\left(10^{5}\right)$ to $\mathcal{O}\left(10^{7}\right)$, depending on the Reynolds number in which the simulation will take place, and obviously the available computational 
power. This means, to be able to use ideas from the dynamical system theory [19], which has been widely used, the phase portrait of the system, in which a 3D flow field is represented as a single point, will be of the quoted orders as well. Meaningful visualization of such a high dimensional phase space is not achievable in a direct sense. One of the latest developments, which also arises from dynamical systems theory, is to use unstable periodic solutions to the underlying fluid flow problem, in a modal expansion for spatio-temporally complicated problems [20, 21]. The forerunner of this methodology was the work by Nagata [22], in which a pair of solutions (termed upper and lower branch solutions) evolve to plane Couette flow starting from a solution of Taylor-Couette flow (the wavy vortex solution). Later Waleffe generated families of exact solutions (in the form of equilibria and waves) for Couette and Poiseuille flows at a range of parameters $[23,24,25]$. Wallefe termed these solutions exact coherent structures owing to the fact that these solutions resembled the coherent structures observed in flow simulations and experiments. State-space visualization via the use of these exact coherent structures (or equilibrium solutions), has been successfully applied to Couette flow [26]. By constructing a basis out of the equilibrium solutions, Gibson et. al manage to identify the unstable manifolds embedded in the phase space [26].

As mentioned earlier in literature, transition to turbulence studies in pipe flow generally utilize DNS [27. These DNS are usually implemented via spectral (pseudospectral [28, spectral element [29]) or finite difference methods [30, 31], or a mixture of these two method families. Within the framework of these methods, it is possible to employ solenoidal basis functions in the expansion of velocity. The use of solenoidal functions in fluid mechanics problems appeared earlier in various studies such as [32] in the study of Taylor-Couette flow, and [33, 13] in the study of pipe flow. The convergence properties of expansions in divergence-free functions in the case of Stokes equations along with pressure reconstruction algorithm is studied in [34] and a domain decomposition approach can be found in [35]. Another early formulation of the incompressible fluid flow problems in terms of divergence-free functions can be found in [36]. 


\subsection{Turbulent Drag Reduction}

Reduction of drag in turbulent flows is often desirable, especially in industrial applications, as one of the major differences between laminar flows and turbulent flows is the fact that turbulent flows experience a marked increase in drag, this increase can be a few orders of magnitude depending on the Reynolds number. Therefore reducing drag in a turbulent flow is of paramount importance. There are numerous techniques, both active and passive, employed for drag reduction. Active control within the framework of flow control signifies the usage of energy power sources whereas passive control mechanisms do not involve energy input into the system. Drag reduction due to spanwise wall oscillations is a case of active flow control where the walls bounding the fluid are oscillated resulting in the reorganization of near wall structures and a significant drop in wall shear hence drag. Utilization of wall oscillations was first proposed by Jung et al. [37], whose numerical study predicted approximately $40 \%$ drop in the drag force. Flow in pipes with circular cross sections is commonly encountered and is of obvious technological importance, hence various drag reduction strategies for pipe flow have been object of numerous studies. There are various control strategies for wall bounded flows, which are known to decrease drag, such as spanwise wall oscillations [37, 38, 39, 40, 41, 42] particle addition [43], and phase randomization [44, 45]. Except for phase randomization, the aforementioned methods had been applied successfully to pipe flow.

In this study we employ a solenoidal spectral method to simulate the effects of different approaches to drag reduction at moderate Reynolds numbers, including phase randomization and wall oscillations. We perform direct numerical simulation of Navier-Stokes equations for flows driven by constant mass flux and constant pressure gradient. We perform a comparative analysis on the effectiveness of these methods, together with similarities and differences between their turbulence suppressing mechanisms. The turbulence control mechanisms investigated here, eventhough a comprehensive parametric study is not in the scope of this work, are often classified under the category of flow optimization without optimization [46], this is due to the fact we do not employ sophisticated optimization techniques to attain our objective, i.e. drag reduction. One other advantage of utilizing flow optimization without optimization is that, no sensors 
or measurements are necessary for the success of the control strategies.

\subsubsection{Drag Reduction via Spanwise Wall Oscillations}

Reduction of turbulent activity via spanwise wall oscillations is a well known phenomenon but not all the mechanisms involved are fully understood [47]. In their 1992 study Jung et al. [37, observed that the wall-oscillations caused time-sustained reduction of the streamwise wall-shear stress. They conjectured the drag reduction due to wall oscillations was due to the modification of turbulence producing activity in the near wall region. Further studies, numerical and experimental, also supported this claim that wall oscillations tend to weaken turbulence producing activity in the vicinity of the walls [38, 39, 40, 48, 41, 47, 49]. An experimental study [50], reports the usual reduction in mean velocity gradient near the wall and an upwards shift in the logarithmic velocity profile and conjecture this to be caused by the negative spanwise vorticity in the near-wall region as a result of spanwise wall oscillations. Wall oscillations have been more extensive for channel flow and a scaling parameter has been suggested for this geometry [47]. In pipe flow the main experimental work is [38], and one can cite [40, 42] among the numerical studies (DNS) .

Spanwise oscillations, for pipe flow geometry are oscillations about the pipe axis. There are two parameters for wall oscillations, the oscillation amplitude $A$, which corresponds to the maximum angular displacement, and the frequency of oscillations

$\Omega$. For a pipe oscillating about its axis with amplitude $A$, and frequency $\Omega$ the azimuthal component of velocity at the pipe wall satisfies $v_{\text {osc }}(1, \theta, z, t)=A \sin (\Omega t)$. Spanwise oscillations represent azimuthal forcing on the flow, and do work on the flow. Hence spanwise wall oscillations constitute active flow control.

\subsubsection{Drag Reduction via Phase Randomization}

Turbulent drag reduction via phase randomization was first suggested in a numerical study [44] for channel flow. Later an experimental study was performed, developing a possible real world analogue of phase randomization in simulations [45. It was suggested by this wind tunnel experiment that specially arranged wall protrusions 
resulted in drag reduction by interacting with near wall structures, and certain other arrangements resulted in drag increase, similar to the effects of randomizing higher wave-number modes in numerical studies [45]. The idea behind phase randomization is to disrupt the energy transfer between roll-like structures and wave like structures, which was earlier suggested to cause burst phenomena in plane channel flow, which in turn contribute significantly to turbulence intensity [51].

The idea presented in [44] is to introduce a spatially uniform randomization to Fourier modes throughout the channel, and it was shown that the results are strongly correlated with the period of phase randomization and the mode band selected for the randomization. It was also shown that randomization in higher modes resulted in an increase in turbulent activity. The experimental work carried out to mimic the effects of phase randomizations via designed wall protrusions [45] also showed that certain configurations of protrusions resulted in increased drag (hence increased mixing) instead of drag reduction observed in other cases. Hence, even though phase randomization is an example of passive drag reduction techniques, it is possible to attain a net drag enhancement with this method.

\subsubsection{Other Drag Reduction Methods}

There are numerous methods to facilitate drag reduction. In this section some of these methods, which are not considered in this study, are listed and briefly explained to give the reader a general perspective on known drag reduction techniques.

Introduction of particles into turbulent flows, such as polymer chains is known to produce drag reduction [43]. The existence of a possible link between drag reduction via polymer addition and phase randomization was suggested in [45, but to this day this alleged connection has not been investigated. In addition to polymer addition, use of bubbles and micro-bubbles and gas injection have also shown to be effective [52, 53] in turbulent drag reduction, especially in external flows, where their effects are better investigated in comparison to spanwise oscillations. Further drag reduction methodologies include, but are not limited to, pumping/sucking action through walls [54], use of wavy surfaces [55], and more recently formation of artificial traveling waves for flow stabilization [56]. 


\subsection{Solenoidal Basis Functions}

One of the main difficulties in direct numerical simulation, or any other simulation for that matter, of incompressible fluid flow is the divergence-free condition which arises from the conservation of mass, and the pressure variable which is bereft of an equation of state [57, 58]. The problem with the fact that an equation of state cannot be defined in incompressible flow results in pressure being transformed into a mathematical artifact rather than a relevant physical variable [57, 59]. In incompressible Navier-Stokes equations, hence, the pressure acts, in a sense, like a Lagrangian multiplier which constrains the flow to remain divergence-free (i.e. solenoidal). It must be added however, that the gradient of pressure is a relevant physical quantity (force per unit volume). The most difficult issues in dealing with pressure are specifying boundary conditions and the expensiveness of its computation during simulations [57, 58, 59].

There are various possible formulations of the momentum part of the Navier-Stokes equations, which are mathematically equivalent, whose discretized forms vary significantly in terms of momentum and energy conservation, and handling of various boundary conditions. These different formulations of Navier-Stokes equations, in addition, respond differently to various numerical methods. At least 16 different formulations of the Navier-Stokes equations are possible [58]. In this study, the primitive variable formulation (the so called $\mathbf{u}-P$ equations which were actually recommended by Gresho in his excellent 1991 review [58] as the most favorable form to be utilized) is used, with the conventional form for the viscous term and the advective/convective form for the nonlinear term during computation.

There are various approaches as well, to the problem of the divergence-free condition. Fractional step/time splitting [60], Helmholtz decomposition/projection methods [61, toroidal-poloidal expansion [62, influence matrix method [63, 64], artificial compressibility [65] and penalty methods [66], among others. Some of the aforementioned methods are combined with staggered grid (or MAC) configurations [67], in an effort to decrease the discretization errors. It is also worthwhile to note that, while the methods mentioned above may result in divergence-free velocity fields, the question whether the obtained velocity fields are in fact a solution to the incompressible Navier-Stokes equations is disputed [59]. 
Solenoidal basis functions span the space of functions whose divergence is zero. When these basis functions are employed to expand the velocity field in fluid dynamics problems, the continuity equation is automatically satisfied [32. In addition, usage of the functions renders the solution for the pressure unnecessary [32], resulting in simpler computer code. More importantly by using solenoidal basis functions, the dynamical system obtained is suitable for use in a bifurcation analysis. Thus the need for separate simulation and bifurcation analysis is eliminated. This is a clear advantage over using Karhunen-Loeve (K-L) analysis in conjunction with DNS, however the solenoidal basis functions are not optimal in the energy sense, unlike K-L basis functions (this is only true if the POD is used statically).

The use of solenoidal functions in fluid mechanics problems was proposed, among others, in 32, the convergence properties in case of Stokes equations along with pressure reconstruction algorithm can be found in [34 and a domain decomposition approach can be found in [35, also for a slightly different approach was detailed in [36]. There are also methods to create discrete divergence-free bases (see [68] and the references therein), which could aid in construction of solenoidal bases for arbitrary geometries. A Fourier-Chebyshev based solenoidal method was developed in [69] for pipe flow. This method was utilized to investigate the linear stability properties of pipe flow up to very high centerline Reynolds numbers [69], and the linear stability of pipe in the presence of infinitesimal disturbances upto centerline Reynolds number of $10^{5}$ was shown. A similar method was employed in the investigation of transition to turbulence in pipe flow in a series of studies [70, 71]. A modified version of the same method was used in studying the Takens-Bogdanov bifurcation of traveling waves in pipe flow [72], as well as the investigation of localized transition in pipe flow for very long pipes [73].

\subsection{What is new in this study}

In descending order of novelty, and to the best of authors knowledge, in this study,

- Effects of phase randomization for pipe flow have been analyzed for the first time in literature 
- Effects of phase randomization for constant mass flux flow have been analyzed for the first time in literature,

- Effects of wall oscillations on transient growth of perturbations in the transition region have been investigated for the first time,

- A solenoidal spectral method has been used for simulation of drag reduction for the first time [74].

\subsection{Outline}

The rest of this work is organized in five chapters. Chapter 2 details the governing equations uswd and the scalings employed, details about our numerical method is presented in chapter 3 , turbulence statistics at reference Reynolds numbers are presented in chapter 4 for constant pressure and mass flux driven flows. Chapter 5 presents the simulation of drag reduction via spanwise wall oscillations and phase randomization. Finally, Section 6, summarizes our findings and our comments on the strengths and weaknesses of the numerical method as well as the effectiveness of drag reduction methodologies employed. 


\section{CHAPTER 2}

\section{PIPE FLOW}

Turbulence is the graveyard of theories.

H. W. Liepmann

\subsection{Governing Equations}

The widely accepted mathematical model for fluid flow is the set of equations known as Navier-Stokes equations. Although existence and uniqueness properties of this set of equations are essentially unknown in three space dimensions, except for some trivial cases, Navier-Stokes equations are regarded as being an accurate mathematical model for fluid flow. It is also universally agreed upon that N-S equations are adequate in describing turbulent flow phenomena [2, 3]. For the presented study we use the unsteady incompressible NS equations in cylindrical coordinates as our mathematical model. The dimensional, unsteady, incompressible N-S equations in polar coordinates, in the absence of body forces, are given by (2.1).

$$
\begin{aligned}
\partial_{t} \mathbf{u}+(\mathbf{u} \cdot \nabla) \mathbf{u} & =-\frac{1}{\rho} \nabla p+\nu \Delta \mathbf{u} \\
\nabla \cdot \mathbf{u} & =0
\end{aligned}
$$

where, $\nu$ is the kinematic viscosity of the fluid. 


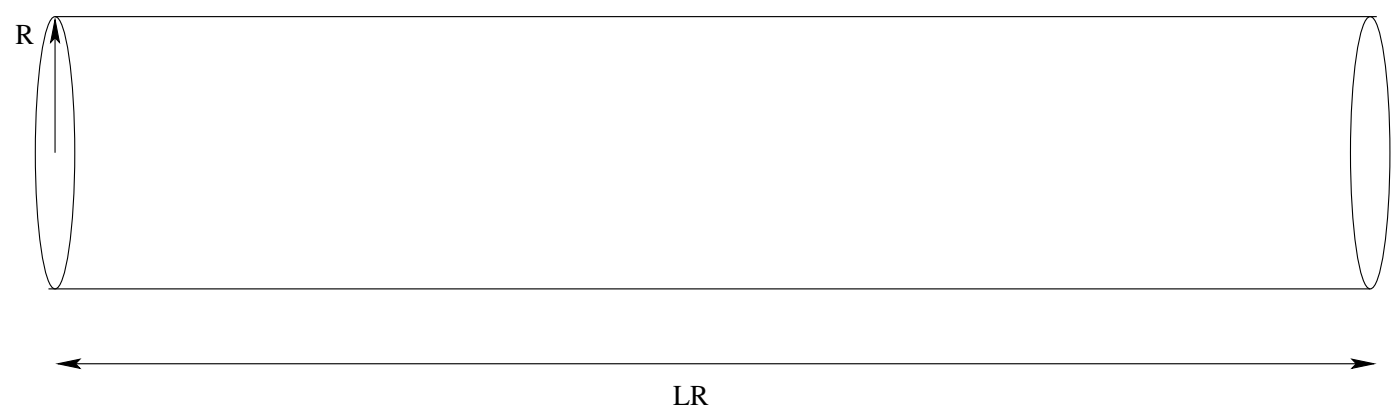

Figure 2.1: Pipe flow geometry

\subsubsection{Non-dimensionalization}

Non-dimensionalization in fluid mechanics is a well known, and almost universally applied technique, which enables a drastic reduction in the number of independent parameters for fluid flow. In the case of incompressible viscous flow, which is the working assumption of this thesis, all the parameters are reduced to the well known Reynolds number. This is a significant advantage for comparing of different works, both experimental and numerical, and decreases the search in the parameter space a few orders of magnitude. Buckhingam- $\Pi$ Theorem is the root of non-dimensionalization 75], which provides a systematic way of constructing non-dimensional parameters. Osborne Reynolds states this as [12],

"...[T]hat the general character of the motion of fluids in contact with solid surfaces depends on the relation between a physical constant of the fluid and the product of the linear dimensions of the space occupied by the fluid and the velocity.",

where he essentially describes what is now known as the Reynolds number. For the non-dimensionalization procedure, first a number of reference quantities must be defined. With the choice of length scale $[L]=l_{\text {ref }}$, velocity scale $[V]=u_{r e f}$, and the corresponding time scale $[T]=\frac{\left[l_{r e f}\right]}{\left[u_{r e f}\right]}$, together with a scaling for the pressure in the 
form $p=p^{*} \rho u_{r e f}^{2}$, the scaled (non-dimensionalized) operators and the variables read,

$$
\begin{aligned}
u^{*} & =\frac{u}{u_{r e f}} \\
p^{*} & =\frac{p}{\rho u_{r e f}^{2}} \\
\nabla & =\frac{\nabla^{*}}{l_{r e f}} \\
\Delta & =\frac{\Delta^{*}}{l_{r e f}^{2}},
\end{aligned}
$$

where the superscript ${ }^{*}$ is used to denote the scaled variables and operators. When the scalings and the scaled operators (2.2) are applied to (2.1), one obtains (2.3) as,

$$
\begin{aligned}
\frac{u_{r e f}^{2}}{l_{r e f}} \partial_{t} \mathbf{u}^{*}+\left(u_{r e f} \mathbf{u}^{*} \cdot \frac{1}{l_{r e f}} \nabla^{*}\right) u_{r e f} \mathbf{u}^{*} & =-\frac{u_{r e f}^{2}}{l_{r e f}} \nabla^{*} p^{*}+\frac{u_{r e f}}{l_{r e f}^{2}} \nu \Delta^{*} \mathbf{u}^{*} \\
\nabla^{*} \cdot \mathbf{u}^{*} & =0 .
\end{aligned}
$$

rearranging (2.3), and dropping the superscript *, as is customary, results in,

$$
\begin{aligned}
\partial_{t} \mathbf{u}+(\mathbf{u} \cdot \nabla) u_{r e f} \mathbf{u} & =-\nabla p+\frac{\nu}{u_{r e f} l_{r e f}} \Delta \mathbf{u} \\
\nabla \cdot \mathbf{u} & =0 .
\end{aligned}
$$

Hence, the governing system of unsteady incompressible Navier-Stokes (N-S) equations in polar coordinates, when non-dimensionalized with a reference velocity $u_{r e f}$ and reference length $l_{r e f}$, with the introduction of the Reynolds number $\operatorname{Re}_{r e f}=u_{r e f} l_{r e f} / \nu$, can be written in the form shown in the following form,

$$
\begin{aligned}
\partial_{t} \mathbf{u}+(\mathbf{u} \cdot \nabla) \mathbf{u} & =-\nabla p+\frac{1}{\operatorname{Re}_{\mathrm{ref}}} \Delta \mathbf{u} \\
\nabla \cdot \mathbf{u} & =0,
\end{aligned}
$$

where, $\mathbf{u}=(u, v, w)$, denotes the velocity field with radial, azimuthal and axial components, respectively and $p=p^{\prime}+\mathrm{G} z$ the pressure. The symbol G in Equation (2.5) represents the magnitude of the mean non-dimensional pressure gradient along the pipe axis, $\mathbf{e}_{z}$. Various forms $\mathrm{G}$ assumes for different scalings commonly used are given in $(2.6),(2.8)$, 
When the centerline velocity, $U_{c l}$, of laminar flow, is selected as the velocity scale, and the length scale is set to be the pipe radius $R$ (also called as macro scaling, and the resulting units are called macro units),

$$
\operatorname{Re}_{\mathrm{cl}}=\frac{\Pi R^{3} \rho}{4 \mu^{2}} \quad \text { and } \quad G=\frac{4}{\operatorname{Re}_{\mathrm{cl}}},
$$

where $\Pi$ is the dimensional driving pressure gradient, $\rho$ the density and $\mu$ is the dynamic viscosity. On the other hand, when the velocity scale is the friction velocity and the length scale is again the pipe radius (also called friction or inner scaling and the resulting units are called friction units or inner units),

$$
\operatorname{Re}_{\tau}=\frac{u_{\tau} R}{\nu} \text { and } G=2,
$$

where $u_{\tau}=\sqrt{\tau_{w} / \rho}$ is the wall friction velocity and $\tau_{w}$ the wall shear stress. For friction scaling the mean (driving) pressure gradient is independent of the Reynolds number, and is fixed.

Another scaling arises, when the bulk mean velocity is kept constant,

$$
U_{B}=\frac{1}{\pi} \int_{0}^{2 \pi} \int_{0}^{1} w r d r d \theta
$$

and the pressure gradient varies in time in order to keep the bulk velocity constant. In this case the velocity scale becomes $2 U_{B}$, which corresponds to the maximum (which is locates at the pipe center) velocity of the laminar flow. Originally the Reynolds number was defined under this scaling [12] and takes the form,

$$
\operatorname{Re}=\frac{2 U_{B} R}{\nu} \text { and } G=G(t)
$$

where $G(t)$ is the time varying pressure gradient required to keep the bulk velocity constant. In this study the constant pressure gradient and constant mass flux (constant bulk mean velocity) cases will be investigated.

The solution domain of the problem at hand is, in all of the scalings used for computation( namely (2.6), (2.8), (2.7) ),

$$
r \in(0,1], \quad \theta \in[0,2 \pi), \quad z \in[0, L / R) .
$$

In this domain, the flow is naturally periodic along azimuthal $(\theta)$ direction and taken to be periodic along the axial $(z)$ direction. The no-slip condition $(2.9)$,

$$
\mathbf{u}(1, \theta, z, t)=\mathbf{0}
$$


is imposed at the pipe wall.

In addition to the presented scalings, it is customary to present some results in the so called wall-units. The wall-units or the wall scaling can be defined with the velocity scaling selected similar to (2.7), as $u_{\tau}$, with a length scale $\nu / u_{\tau}$ (which is also called as the viscous thickness), and subsequently a time scale given by $\nu / u_{\tau}^{2}$. In order to relate the time in friction units to time in wall units, consider an arbitrary dimensional time $T$, with its value in friction scaling $T^{\tau}$, and the relevant value in wall units $T^{+}$,

$$
\begin{gathered}
T=\frac{T^{+} \nu}{u_{\tau}^{2}}, \\
T=\frac{T^{\tau} R}{u_{\tau}},
\end{gathered}
$$

Hence,

$$
\frac{T^{\tau} R}{u_{\tau}}=\frac{T^{+} \nu}{u_{\tau}^{2}}
$$

So the relation becomes,

$$
T^{\tau}=\frac{T^{+} \nu}{u_{\tau} R}
$$

From the definition of friction Reynolds number,

$$
\operatorname{Re}_{\tau}=\frac{u_{\tau} R}{\nu}
$$

finally one gets,

$$
T^{\tau}=\frac{T^{+}}{R e_{\tau}}
$$

Similarly for the radial coordinate,

$$
r^{+}=\operatorname{Re}_{\tau}(1-r)
$$

as the radial coordinate in wall units is customarily measured starting from the wall. Some other useful relations between various scaled quantities in different unit systems are as follows. 
The bulk velocity in wall or friction units, in the case of bulk scaling, is also given by,

$$
u_{B}^{+}=\sqrt{\frac{1}{2 G_{B}}}
$$

where $G_{B}$ is the pressure gradient required to drive the flow with constant mass flux in bulk scaling [76]. In addition, the friction Reynolds number in this scaling can be calculated by,

$$
R e_{\tau}=\sqrt{\operatorname{Re} \frac{\partial \bar{w}}{\partial r}}
$$

yet another useful expression which relates the bulk flow velocity in friction scaling directly to radial derivative of the mean velocity profile in bulk scaling is ,

$$
\left(u_{B} / u_{\tau}\right)^{2}=R e /\left(4 \frac{\partial \bar{w}}{\partial r}\right) .
$$

\subsubsection{Laminar Flow}

As a final derivation of the laminar flow in the case of pipe flow is detailed below.In order to obtain the laminar solution to N-S equations for pipe flow, one assumes the flow to be steady, velocity be oriented along the axis of the pipe, and be independent of azimuthal and radial directions, these can be written as, denoting the laminar solution with subscript $l$,

$$
\begin{aligned}
\mathbf{u}_{l} & =\left(0,0, w_{l}\right) \\
\frac{\partial w_{l}}{\partial t} & =0 \\
\frac{\partial w_{l}}{\partial r} & =0 \\
\frac{\partial w_{l}}{\partial \theta} & =0 .
\end{aligned}
$$

With the simplifications given in (2.10), the first two components of (2.1) becomes,

$$
\begin{aligned}
& \frac{\partial p}{\partial r}=0 \\
& \frac{\partial p}{\partial \theta}=0
\end{aligned}
$$


this implies, $p=\Pi \hat{z}+c$, where $\mathrm{c}$ is an arbitrary constant and $\Pi$ is the pressure gradient along the axis of the pipe, hence the N-S equations are reduced to,

$$
w^{\prime \prime}+\frac{w^{\prime}}{r}=-\frac{\Pi}{\rho \nu},
$$

where the superscript ' denotes derivative with respect to $r$. This second order ODE can be written as,

$$
\frac{1}{r} \frac{d}{d r}\left(r w^{\prime}\right)=-\frac{\Pi}{\rho \nu}
$$

Integrating once yields,

$$
r w^{\prime}=\frac{\Pi r^{2}}{2 \rho \nu}+c_{1},
$$

where $c_{1}$ is the integration constant. Integrating once more, one obtains,

$$
w=\frac{\Pi r^{2}}{4 \rho \nu}+c_{1} \ln (r)+c_{2} .
$$

For the solution to be non-singular at $r=0$, the integration constant $c_{1}$ must be equal to zero, applying the no-slip boundary condition at the wall (i.e $\mathrm{r}=1$ ), results in the well known parabolic velocity profile,

$$
w=\frac{\Pi r^{2}}{4 \rho \nu}\left(R^{2}-r^{2}\right),
$$

where $R$ is the radius of the pipe, and $r$ is the radial variable ranging from $[0-\mathrm{R}]$. Under the scalings in (2.6) and (2.6), the laminar flow becomes $\mathbf{u}_{l}=\left(0,0,1-r^{2}\right)$.

The present numerical approach for solving (2.5) is essentially a Galerkin type scheme involving the solenoidal basis functions satisfying the boundary conditions for the expansion of the velocity field and the solenoidal dual basis functions for the construction of the projection space. In the process, the pressure variable in (2.5) is eliminated. The next section details this approach. 


\title{
CHAPTER 3
}

\section{THE NUMERICAL METHOD}

\begin{abstract}
There appeared to be two ways of proceeding-the one theoretical, the other practical. The theoretical method involved the integration of the equations for unsteady motion in a way that had not been accomplished and which, considering the general intractability of the equations, was not promising.
\end{abstract}

Osborne Reynolds [12]

In this chapter, the numerical method developed in oder to solve Equation (2.5) is presented. The method employs solenoidal bases for the space discretization and an IMEX (semi-implicit) scheme for marhing in time. A similar approach utilizing the solenoidal basis functions for the study of turbulence transition and transient growth of perturbations for pipe flow are detailed in [77, 13]. In the aformentioned works, the construction of the bases is facilitated by Chebyshev polynomials. In this work, however, Legendre polynomials are used in the formulation of the solenoidal basis functions and their duals. This is preferred for their performance in the numerical evaluation of the inner product integrals and for the resulting form of the solenoidal basis functions.

This study employes two solenoidal basis function sets, namely the pysical (trial) basis functions, and the dual (test) basis functions. The function sets have different uses. The physical basis functions are used to expand the velocity field, whereas the dual basis functions define the space unto which the governing equations are projected.

To summarize the workings of the numerical method, first the velocity field is ex- 


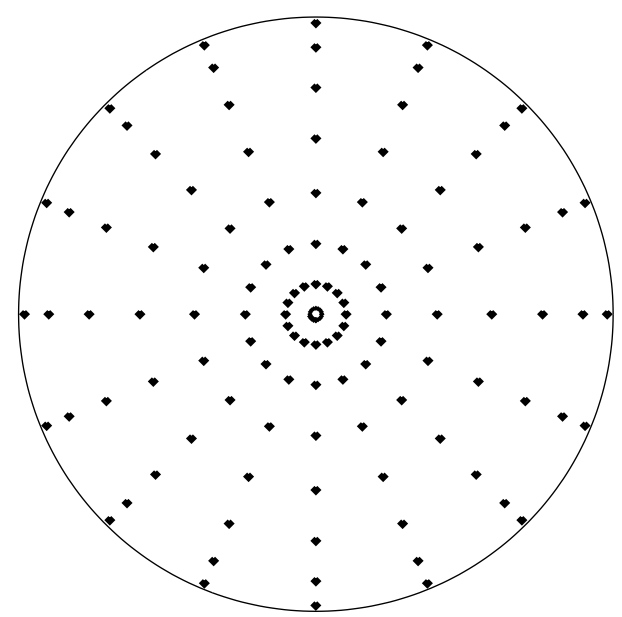

(a) Clustered grid

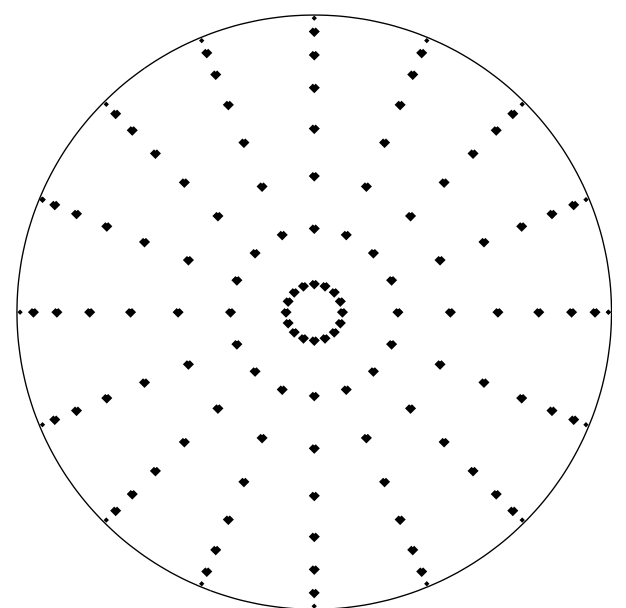

(b) Non-clustered grid

Figure 3.1: Comparison of clustered and non-clustered grids for 8 radial grid points

panded in terms of the first set of solenoidal bases (physical basis functions), the expression for velocity is then substituted into the governing equations and the resulting residual equations are projected onto the second set of solenoidal bases (dual basis functions), with the aim of eliminating the residual error. Following the projection, space dependence in the problem and the pressure term are eliminated. This results in a system of differantial algebraic equations (DAEs), for the time dependent expansion coefficients [78, 79], which is continous in time. The resulting system of DAEs then is discretized in time using an semi-implicit (or IMEX) scheme.

Discretization in space is handled by equidistant grids in two dimensions and a Legendre grid along the other space dimension. Equally spaced grids are used along the homogenous (periodic), azimuthal and axial, directions, a Gauss-Legendre grid is used to dicretize the radial direction. Special attention has to be paid to the distribution of grid points in the vicinity of the pipe center $r=0$, as one would like to avoid the singularity at the pipe center and also to avoid excessive grid crowding (clustering) near the origin [80]. The grid clustering phenomena in polar geometries is illustrated in Fig. 3.1. 


\subsection{Basis Functions}

There are two sets of conditions to be satisfied by the physical $\Psi$ and the dual $\Phi$ basis function families. The first one is the solenoidal condition, i.e. the basis functions should be divergence free,

$$
\begin{aligned}
& \nabla \cdot \Psi=0 \\
& \nabla \cdot \Phi=0 .
\end{aligned}
$$

It is worthwhile to note that the above formulation for the dual bases is only valid, if the dual bases are constructed using Legendre polynomials, which are orthogonal with respect to weight unity. If for example Chebyshev polynomials are used, as in [13], the condition becomes $\nabla \cdot(\omega \Phi)=0$, where $\omega=1 /\left(\sqrt{1-r^{2}}\right)$, which further complicates the construction of the solenoidal bases. In addition to the above condition, the physical basis functions are required to satisfy the no-slip boundary conditions prescribed in (2.9), On the other hand, the dual bases are only required the satisfy the condition of the vanishing flux through the walls in order to enable the elimination of the pressure term $\nabla p^{\prime}$ from the equations (2.5) [32]. These additional conditions are

$$
\begin{aligned}
\Psi(1, \theta, z) & =0 \\
\Phi(1, \theta, z) \cdot \mathbf{e}_{r} & =0 .
\end{aligned}
$$

In the pipe geometry, while the flow in the azimuthal direction is naturally periodic, the flow in the axial direction is taken to be periodic as it is a common practice in literature. This leads to the use of Fourier representation along the corresponding $\theta, z$ coordinates and the basis functions take the following form,

$$
\begin{aligned}
& \Psi_{l n m}^{(1,2)}(r, \theta, z)=e^{i(n \theta+2 \pi l z / Q)} \mathcal{V}_{l n m}^{(1,2)}(r) \\
& \Phi_{l n m}^{(1,2)}(r, \theta, z)=e^{i(n \theta+2 \pi l z / Q)} \overline{\mathcal{V}}_{l n m}^{(1,2)}(r) .
\end{aligned}
$$

The superscripts on the basis functions signify the sufficiency of the two degrees of freedom in representing the three components of a solenoidal velocity field as the continuity equation provides the connection between the components. In the Fourier representation, the three components $\left(V_{r}, V_{\theta}, V_{z}\right)$ of $\mathbf{V}$ and $\overline{\mathbf{V}}$ are required to satisfy 
the reduced form of the continuity equation,

$$
D_{+} \mathcal{V}_{r}+\frac{i n}{r} \mathcal{V}_{\theta}+i l \mathcal{V}_{z}=0
$$

where $D_{+}=D+\frac{1}{r}$ and $D=d / d r$. The basis functions are then constructed to satisfy $(3.2)$ and $(3.3)$. The regularity requirement in the vicinity of the pipe center 81,82 and the use of Gauss-Legendre quadrature $\left(\omega_{k}, r_{k}\right)$ [83] in the numerical evaluation of the inner product integrals,

$$
\begin{aligned}
(\overline{\mathcal{V}}, \mathcal{V})=\int_{0}^{1} \overline{\mathcal{V}}(r)^{*} \cdot \mathcal{V}(r) r d r & =\frac{1}{2} \int_{-1}^{1} \overline{\mathcal{V}}(r)^{*} \cdot \mathcal{V}(r) r d r \\
& =\frac{1}{2} \sum_{k=0}^{K} \overline{\mathcal{V}}\left(r_{k}\right)^{*} \cdot \mathcal{V}\left(r_{k}\right) r_{k} \omega_{k}
\end{aligned}
$$

impose additional requirements on the basis functions such as the evenness of the integrands.

\subsubsection{Construction of the Bases}

In this section the construction of the bases is detailed. We start with the continuity equation transformed to Fourier space (3.3). For various values of wave number and other constraints, such as the analicity and the parity of the functions are used to construct the bases.

Case $\mathbf{I}, l \neq 0 n=0, \Rightarrow D_{+} \mathcal{V}_{r}+i l \mathcal{V}_{z}=0$

$$
\begin{array}{ll}
\text { Physical "1-basis": } & \text { Physical "2-basis": } \\
\mathcal{V}^{(1)}=\left(\begin{array}{c}
0 \\
\mathcal{V}_{\theta} \\
0
\end{array}\right) & \mathcal{V}^{(2)}=\left(\begin{array}{c}
-i l \mathcal{V}_{r} \\
0 \\
D_{+} \mathcal{V}_{r}
\end{array}\right)
\end{array}
$$

where $\mathcal{V}_{r}=r f_{E}(r), \mathcal{V}_{\theta}=r g_{E}(r)$ for some $f_{E}, g_{E}$ analytic and even functions for the analticity of the flow field. The boundary conditions,

$$
\mathcal{V}_{r}(r=1)=\mathcal{V}_{\theta}(r=1)=0,
$$


on the wall give

$$
\mathcal{V}_{r}=r\left(1-r^{2}\right)^{2} P_{2 m} \quad \text { and } \quad \mathcal{V}_{\theta}=r\left(1-r^{2}\right) P_{2 m}
$$

The dual is constructed similarly, only that the boundary conditions needed for the pressure term to vanish is $\tilde{\mathcal{V}}_{r}(r=1)=0$. Thus

$$
\tilde{\mathcal{V}}_{r}=\left(1-r^{2}\right) P_{2 m} \quad \text { and } \quad \tilde{\mathcal{V}}_{\theta}=P_{2 m},
$$

thus the dual reads,

$$
\begin{array}{lr}
\text { Dual "1-basis": } & \text { Dual "2-basis": } \\
\tilde{\mathcal{V}}^{(1)}=\left(\begin{array}{c}
0 \\
\tilde{\mathcal{V}}_{\theta} \\
0
\end{array}\right) & \tilde{\mathcal{V}}^{(2)}=\left(\begin{array}{c}
-i l \tilde{\mathcal{V}}_{r} \\
0 \\
D_{+} \tilde{\mathcal{V}}_{r}
\end{array}\right)
\end{array}
$$

Another criteria in selecting the form of the dual basis is the parity of the following intergrands,

$$
\int_{0}^{1} a^{*} \cdot b r d r=\frac{1}{2} \int_{-1}^{1} a^{*} \cdot b r d r
$$

arising in the projection of the governing equations onto the dual space. The integrand needs to be even or $a^{*} \cdot b$ needs to be odd.

Case II $, l \neq 0 n \neq 0, \Rightarrow D_{+} \mathcal{V}_{r}+\frac{i n}{r} \mathcal{V}_{\theta}+i l \mathcal{V}_{z}=0$

Writing $\mathcal{V}_{\theta}$ in terms of the other velocity components using continuity equation as a constraint,

$$
\begin{aligned}
\mathcal{V} & =\left(\begin{array}{c}
\mathcal{V}_{r} \\
\frac{r}{i n}\left(-D_{+} \mathcal{V}_{r}-i l \mathcal{V}_{z}\right) \\
\mathcal{V}_{z}
\end{array}\right) \\
& =\left(\begin{array}{c}
\mathcal{V}_{r} \\
-\frac{r}{i n} D_{+} \mathcal{V}_{r} \\
0
\end{array}\right)+\left(\begin{array}{c}
0 \\
-\frac{r l}{n} \mathcal{V}_{z} \\
\mathcal{V}_{z}
\end{array}\right) \\
& =\left(\begin{array}{c}
-i n \mathcal{V}_{r} \\
r D_{+} \mathcal{V}_{r} \\
0
\end{array}\right)+\left(\begin{array}{c}
0 \\
-i r l \mathcal{V}_{z} \\
i n \mathcal{V}_{z}
\end{array}\right)=\left(\begin{array}{c}
-i n \mathcal{V}_{r} \\
D\left(r \mathcal{V}_{r}\right) \\
0
\end{array}\right)+\left(\begin{array}{c}
0 \\
-i r l \mathcal{V}_{z} \\
i n \mathcal{V}_{z}
\end{array}\right)
\end{aligned}
$$


Analycity requirement for $n \neq 0$ [81,

$$
u_{r}=r^{|n|-1} f_{E} \quad u_{\theta}=r^{|n|-1} g_{E} \quad u_{z}=r^{|n|} h_{E},
$$

dictates that,

$$
\mathcal{V}_{r}=r^{|n|-1}\left(1-r^{2}\right)^{2} P_{2 m} \quad \mathcal{V}_{z}=r^{|n|}\left(1-r^{2}\right) P_{2 m}
$$

Also note that the equivalence of index negation and complex conjugacy, i.e.,

$$
\mathcal{V}(-l,-n, r)=\mathcal{V}^{*}(l, n, r),
$$

is satisfied. In order to construct the dual basis, the parity is considered, if $n$ is odd,

$$
\mathcal{V}^{1}=\left(\begin{array}{c}
\text { even } \\
\text { even } \\
0
\end{array}\right) \quad \mathcal{V}^{2}=\left(\begin{array}{c}
0 \\
\text { even } \\
\text { odd }
\end{array}\right) \Rightarrow \overline{\mathcal{V}}^{1}=\left(\begin{array}{c}
\text { odd } \\
\text { odd } \\
0
\end{array}\right) \quad \overline{\mathcal{V}}^{2}=\left(\begin{array}{c}
0 \\
\text { odd } \\
\text { even }
\end{array}\right)
$$

Hence for this case, the dual base read

$$
\overline{\mathcal{V}}=\left(\begin{array}{c}
-i n \overline{\mathcal{V}}_{r} \\
D\left(r \overline{\mathcal{V}}_{r}\right) \\
0
\end{array}\right)+\left(\begin{array}{c}
0 \\
-i l r \overline{\mathcal{V}}_{z} \\
i n \overline{\mathcal{V}}_{z}
\end{array}\right)
$$

using the only boundary condition, $\overline{\mathcal{V}}_{r}(r=1)=0$, yields

$$
\overline{\mathcal{V}}_{r}=r\left(1-r^{2}\right) P_{2 m} \quad \overline{\mathcal{V}}_{z}=P_{2 m} .
$$

In case $n$ is even, then the the dual basis becomes,

$$
\begin{gathered}
\mathcal{V}^{1}=\left(\begin{array}{c}
\text { odd } \\
\text { odd } \\
0
\end{array}\right) \quad \mathcal{V}^{2}=\left(\begin{array}{c}
0 \\
\text { odd } \\
\text { even }
\end{array}\right) \Rightarrow \overline{\mathcal{V}}^{1}=\left(\begin{array}{c}
\text { even } \\
\text { even } \\
0
\end{array}\right) \quad \overline{\mathcal{V}}^{2}=\left(\begin{array}{c}
0 \\
\text { even } \\
\text { odd }
\end{array}\right), \\
\overline{\mathcal{V}}=\left(\begin{array}{c}
-i n \overline{\mathcal{V}}_{r} \\
\left.D(r) \overline{\mathcal{V}}_{r}\right) \\
0
\end{array}\right)+\left(\begin{array}{c}
0 \\
-i l r \overline{\mathcal{V}}_{z} \\
i n \overline{\mathcal{V}}_{z}
\end{array}\right)
\end{gathered}
$$

with,

$$
\overline{\mathcal{V}}_{r}=\left(1-r^{2}\right) P_{2 m} \quad \overline{\mathcal{V}}_{z}=r P_{2 m} .
$$


Case III, $l=n=0, \Rightarrow D_{+} \mathcal{V}_{r}=0$

In this case, noting that $\mathcal{V}_{r}=0$ is a solution,

$$
\mathcal{V}=\left(\begin{array}{c}
0 \\
\mathcal{V}_{\theta} \\
0
\end{array}\right)+\left(\begin{array}{c}
0 \\
0 \\
\mathcal{V}_{z}
\end{array}\right) \quad \text { with } \quad \mathcal{V}_{\theta}(r=1)=\mathcal{V}_{z}(r=1)=0
$$

Analycity requires $\mathcal{V}_{\theta}=r g_{E}, \mathcal{V}_{z}=h_{E}$, thus

$$
\mathcal{V}_{\theta}=r\left(1-r^{2}\right) P_{2 m} \quad \mathcal{V}_{z}=\left(1-r^{2}\right) P_{2 m}
$$

The dual basis in this case is,

$$
\overline{\mathcal{V}}=\left(\begin{array}{c}
0 \\
\overline{\mathcal{V}}_{\theta} \\
0
\end{array}\right)+\left(\begin{array}{c}
0 \\
0 \\
\overline{\mathcal{V}}_{z}
\end{array}\right)
$$

where,

$$
\overline{\mathcal{V}}_{\theta}=P_{2 m} \quad \overline{\mathcal{V}}_{z}=r P_{2 m}
$$

Case IV $, l=0, n \neq 0, \Rightarrow D_{+} \mathcal{V}_{r}+\frac{i n}{r} \mathcal{V}_{\theta}=0$

$$
\begin{aligned}
\mathcal{V} & =\left(\begin{array}{c}
\mathcal{V}_{r} \\
-\frac{r}{i n} D_{+} \mathcal{V}_{r} \\
\mathcal{V}_{z}
\end{array}\right)=\left(\begin{array}{c}
\mathcal{V}_{r} \\
-\frac{r}{i n} D_{+} \mathcal{V}_{r} \\
0
\end{array}\right)+\left(\begin{array}{c}
0 \\
0 \\
\mathcal{V}_{z}
\end{array}\right) \\
& =\left(\begin{array}{c}
-i n \mathcal{V}_{r} \\
i n D\left(r \mathcal{V}_{r}\right) \\
0
\end{array}\right)+\left(\begin{array}{c}
0 \\
0 \\
i n \mathcal{V}_{z}
\end{array}\right),
\end{aligned}
$$

again considering the parity, for $n$ odd,

$$
\mathcal{V}^{1}=\left(\begin{array}{c}
\text { even } \\
\text { even } \\
0
\end{array}\right) \quad \mathcal{V}^{2}=\left(\begin{array}{c}
0 \\
0 \\
\text { odd }
\end{array}\right) \Rightarrow \overline{\mathcal{V}}^{1}=\left(\begin{array}{c}
\text { odd } \\
\text { odd } \\
0
\end{array}\right) \quad \overline{\mathcal{V}}^{2}=\left(\begin{array}{c}
0 \\
0 \\
\text { even }
\end{array}\right)
$$

this implies, 


$$
\overline{\mathcal{V}}=\left(\begin{array}{c}
-i n \overline{\mathcal{V}}_{r} \\
D\left(r \overline{\mathcal{V}}_{r}\right) \\
0
\end{array}\right)+\left(\begin{array}{c}
0 \\
0 \\
i n \overline{\mathcal{V}}_{z}
\end{array}\right)
$$

where $\overline{\mathcal{V}}_{r}=r\left(1-r^{2}\right) P_{2 m}$ and $\overline{\mathcal{V}}_{z}=P_{2 m}$. Similarly for $n$ even,

$$
\mathcal{V}^{1}=\left(\begin{array}{c}
\text { odd } \\
\text { odd } \\
0
\end{array}\right) \quad \mathcal{V}^{2}=\left(\begin{array}{c}
0 \\
0 \\
\text { even }
\end{array}\right) \quad \Rightarrow \quad \overline{\mathcal{V}}^{1}=\left(\begin{array}{c}
\text { even } \\
\text { even } \\
0
\end{array}\right) \quad \overline{\mathcal{V}}^{2}=\left(\begin{array}{c}
0 \\
0 \\
\text { odd }
\end{array}\right)
$$

this implies,

$$
\overline{\mathcal{V}}=\left(\begin{array}{c}
-i n \overline{\mathcal{V}}_{r} \\
D\left(r \overline{\mathcal{V}}_{r}\right) \\
0
\end{array}\right)+\left(\begin{array}{c}
0 \\
0 \\
i n \overline{\mathcal{V}}_{z}
\end{array}\right)
$$

where $\overline{\mathcal{V}}_{r}=\left(1-r^{2}\right) P_{2 m}$ and $\overline{\mathcal{V}}_{z}=r P_{2 m}$.

\subsection{Projection}

Having constructed the basis functions, the projection procedure is now employed to reduce (2.5) to a dynamical system via the inner product

$$
(\Psi, \Phi)=\int_{0}^{1} r d r \int_{0}^{2 \pi} d \theta \int_{0}^{L} d z\left(\Psi^{*} \cdot \Phi\right)
$$

which reduces to (3.4) due to the orthogonality of Fourier bases. Only the coefficients $a^{(1)}, a^{(2)}$ in the expansion of the velocity field,

$$
\mathbf{u}(r, \theta, z, t)=\sum_{l=-Q}^{Q} \sum_{n=-N}^{N} \sum_{m=0}^{M} a_{l n m}^{(1)}(t) \Phi_{l n m}^{(1)}(r, \theta, z)+a_{l n m}^{(2)}(t) \Phi_{l n m}^{(2)}(r, \theta, z),
$$

depend on time, and solely on time. Hence, the substitution of (3.6) into (2.5) and the projection of the resulting residual onto the dual bases via (3.5), result in the dynamical system for the expansion coefficients $\mathbf{a}_{l n m}$ in the form,

$$
\mathbf{A}_{l n m^{\prime} m} \dot{\mathbf{a}}_{l n m}=\mathbf{B}_{l n m^{\prime} m} \mathbf{a}_{l n m}-\mathbf{b}_{l n m^{\prime}}
$$




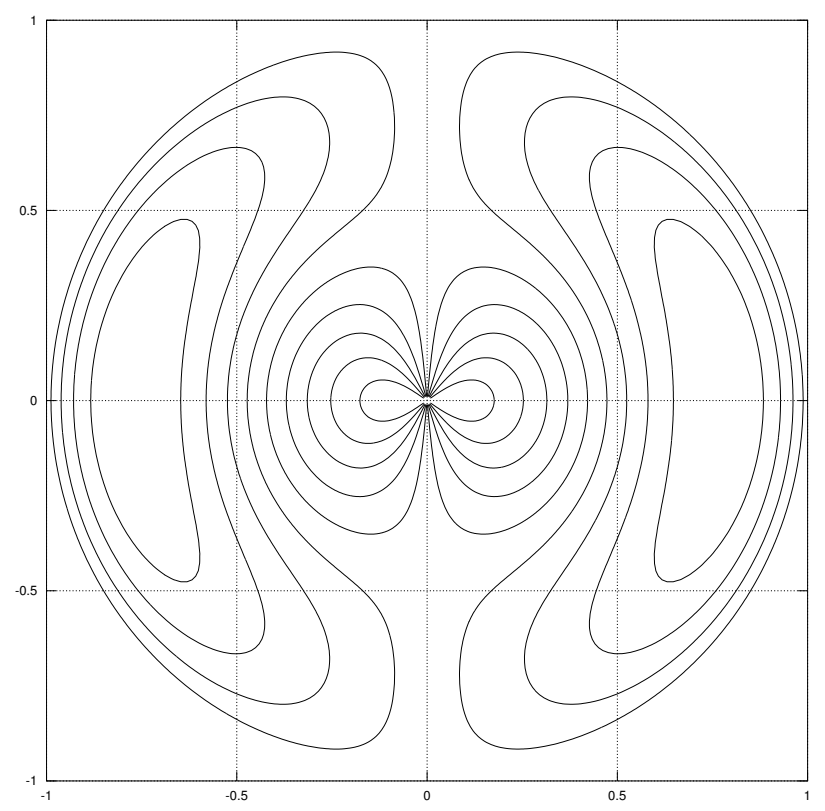

Figure 3.2: Mode $(0,1,0)$ radial contours

where,

$$
\begin{aligned}
\mathbf{B}_{l n m^{\prime} m} & =\left(\Psi_{l n m^{\prime}}, \frac{1}{\operatorname{Re}} \Delta \Phi_{l n m}-\nabla p\right) \\
\mathbf{b}_{l n m^{\prime}} & =\left(\Psi_{l n m^{\prime}},(\mathbf{u} \cdot \nabla) \mathbf{u}\right) .
\end{aligned}
$$

The pressure term $\nabla p$ appears in the expression, but the term $\nabla p^{\prime}$ cancels under projection. The system (3.7) is numerically integrated in time using the 3rd order semi-implicit time-solver,

\subsection{Time Solvers}

The system of nonlinear equations for wave number pairs $(l, n)$, which are given by (3.7) are coupled by the nonlinear terms only. This means after the calculation of the global nonlinear contributions, $b$, each system corresponding to wave number pair $(l, n)$ can be solved independently for the expansion coefficients $a$. This property aids in the parallelization of the code via OpenMP library. Thus at each time step first the nonlinear terms, which correspond to the advective part of the NS equation, 


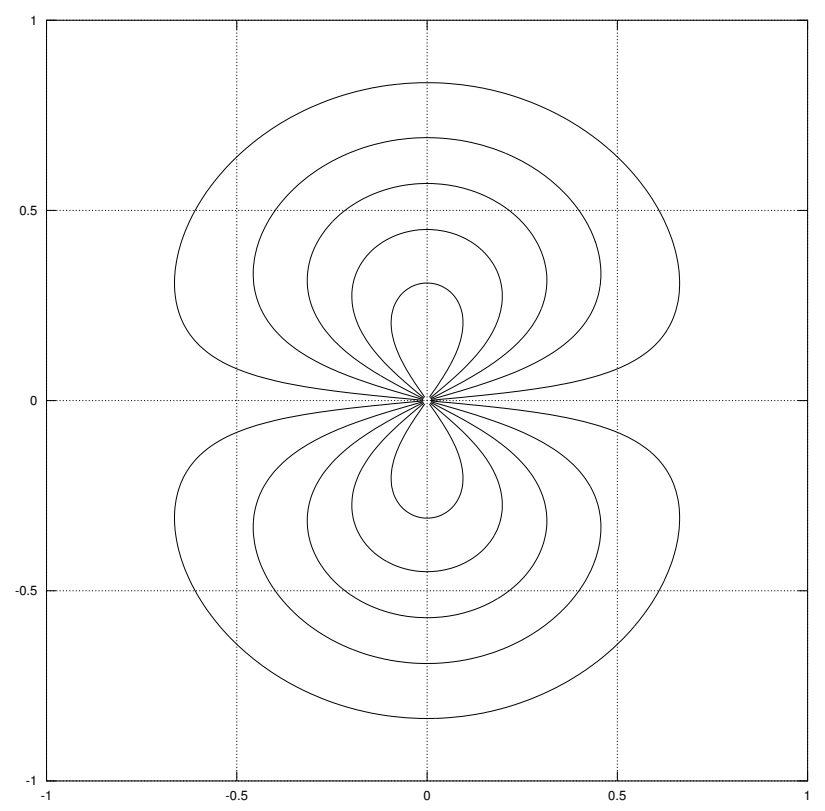

Figure 3.3: Mode $(0,1,0)$ azimuthal contours

are calculated, they are projected onto the dual basis, and the resulting vectors are distributed among the wave number pairs. Following this step, (3.7) is solved for each wave number pair separately, yielding the expansion coefficients.

The time solver selected to solve (3.7), is a semi-implicit scheme in which the linear (diffusive) terms are treated implicitly with backward differentiation of order 4 and the nonlinear (advective) terms are treated with with polynomial extrapolation of order 3 or 4 . Methods of these nature are often called IMEX (implicit-explicit) methods, and our specific choice of time solver is dubbed SBDF [84]. In addition to favorable stability and accuracy properties, IMEX methods are also shown to decrease the aliasing errors arising from employing spectral methods, even though we utilize a fully de-aliased method, this is a notable property.

For the derivation of the $4^{\text {th }}$ order time solver, we first write, 


$$
\begin{aligned}
& \mathbf{A}\left(\frac{25}{12} a^{n+1}-4 a^{n}+3 a^{n-1}-\frac{4}{3} a^{n-2}+\frac{1}{4} a^{n-3}\right)= \\
& \Delta t \mathbf{B} a^{n+1}+\Delta t\left(4 b^{n}-6 b^{n-1}+4 b^{n-2}-b^{n-3}\right),
\end{aligned}
$$

rearranging, we obtain the the time solver also used in [77,

$$
\begin{aligned}
& (25 \mathbf{A}-12 \Delta t \mathbf{B}) a^{(k+1)}= \\
& \mathbf{A}\left(48 a^{(k)}-36 a^{(k-1)}+16 a^{(k-2)}-3 a^{(k-3)}\right) \\
& -\Delta t\left(48 b^{(k)}-72 b^{(k-1)}+48 b^{(k-2)}-12 b^{(k-3)}\right) .
\end{aligned}
$$

This scheme is not self starting, so for the initial steps we use a Runge-Kutta integrator, as in [77. Similarly a third order time integrator can be obtained using backward differences and extrapolation of order three. It is given by,

$$
\begin{aligned}
(11 \mathbf{A}-6 \Delta t \mathbf{B}) a^{(k+1)} & =\mathbf{A}\left(18 a^{(k)}-9 a^{(k-1)}+2 a^{(k-2)}\right) \\
& -\Delta t\left(18 b^{(k)}-18 b^{(k-1)}+6 b^{(k-2)}\right) .
\end{aligned}
$$

While the time integration scheme given by $(3.9)$ is third order, the time step restriction on this method is less severe than the restriction on (3.8), It was also reported that [84, the 3rd order SBDF scheme has the least error among all IMEX methods when it is stable [84]. The only disadvantage in using third order is that the time step restriction is stringent in comparison to second order methods in the low Reynolds number range. For larger integration times and higher resolutions, therefore, the third order scheme is preferable. Therefore the calculations presented in this study are performed using the $3^{\text {rd }}$ order time solver, unless otherwise is stated.

\subsection{The Computer Code}

Our code was first written in octave (octave reference here) for the sake of rapid prototyping, after the initial tests were successfully completed, it was then re-programmed in fortran 95. The GNU fortran compiler was used to compile the code. For the calculation of the fast Fourier transform, the fftw library was used [85, the linear algebra operations were handled using blas, lapack [86]. 
Although one version of the code exists in reality, we depict the flowchart of the code in the constant pressure gradient case in Figure 3.4 and the constant mass flux case in Figure 3.5, for the sake of clarity. The main difference between these two cases is the calculation of the pressure gradient (B.1) and the enforcement of mass flux in the latter case (B.2).

As mentioned earlier, the code uses the OpenMP library for parallelization. This provides a speed-up of 2.7 on a i7 (930) CPU. Although the creation of the matrices appearing in (3.9) are also parallel, the main speed-up is achieved in the time solver. The time solver is parallelized using a OMP PARALLEL DO loop, where the algebraic system (3.9) is divided into four parts. The division is facilitated by the fact that the equations in (3.9) are in essence decoupled after the calculation of the non-linear coefficinets $b^{k}$ for each azimuthal-axial wave number pair $(l, n)$, so they can be handled by different processing cores, the result then is accumulated in the coefficient matrix $a^{k+1}$. In the same manner all the FFT and inverse-FFT calculations are performed in four parts. 


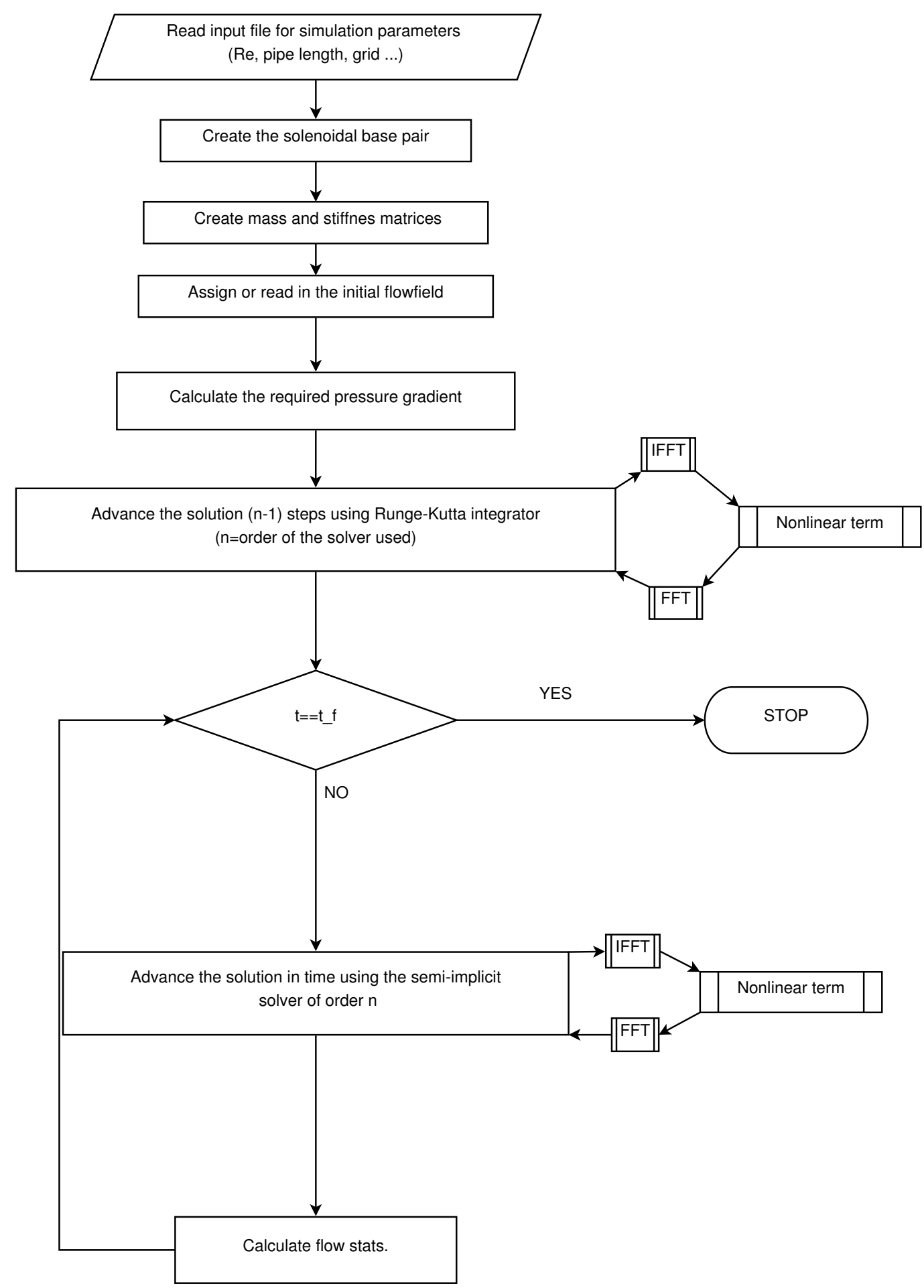

Figure 3.4: Flowchart of the computer code, constant pressure gradient 


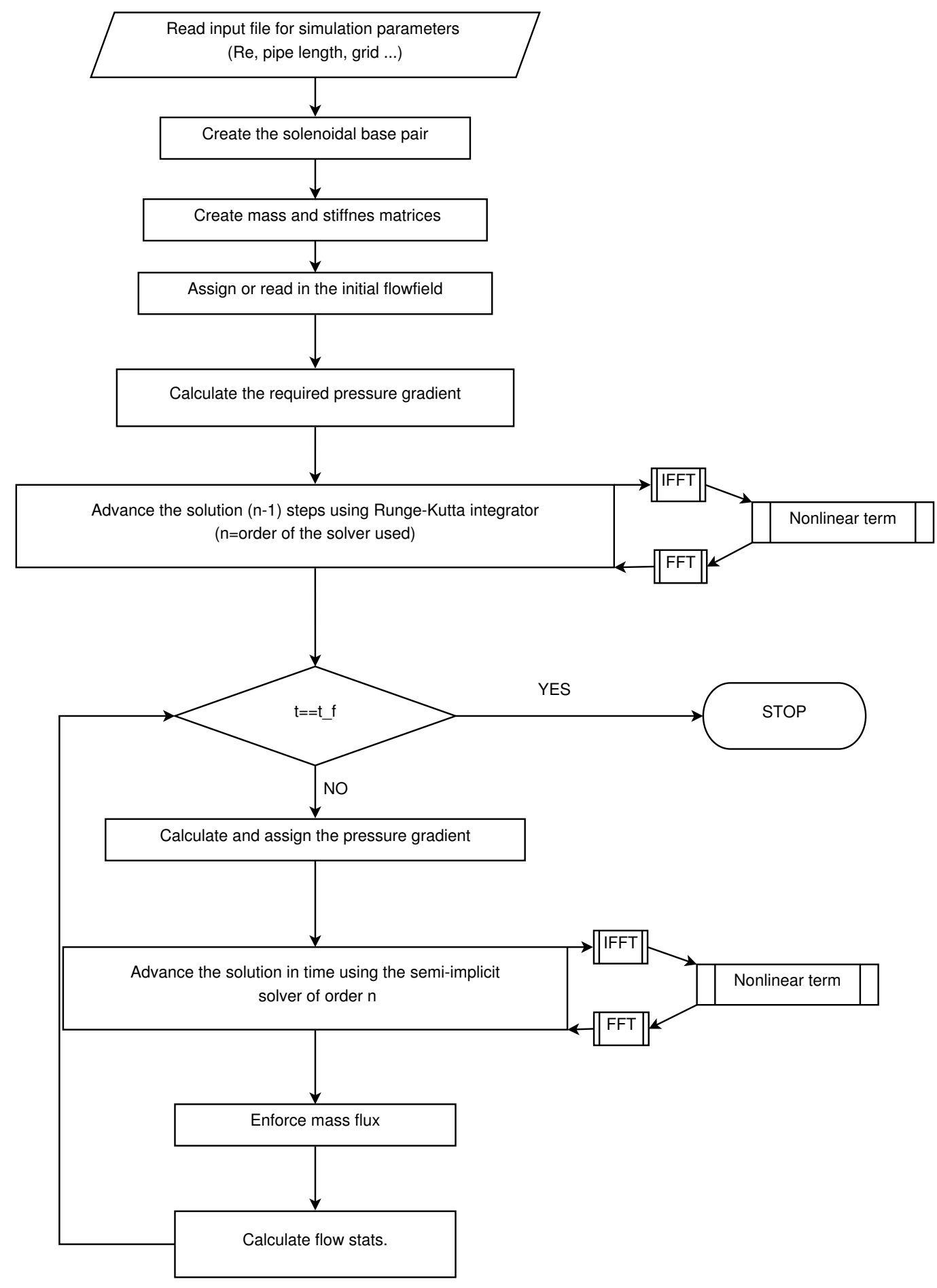

Figure 3.5: Flowchart of the computer code, constant mass flux 


\section{CHAPTER 4}

\section{CODE VALIDATION}

Perfect numbers like perfect men are very rare.

Rene Descartes

\subsection{Laminar Test Case and Linear Stability}

The first test we employ is to see whether our code can reproduce the famous parabolic profile in the laminar region starting from various initial conditions (such as various random flowfields or starting from rest). Our code manages to compute the well known laminar profile in the low Reynolds region starting from various initial conditions up to machine accuracy. For testing purposes, flow starting from rest, and random initial flow fields were employed. It is worthwhile to note that all the compuations involving this phase of the validation process were performed without linearization, i.e. the nonlinear of (2.5), are solved.

As another means of testing our method, we consider the linearization of (2.5) about the laminar base flow, $\mathbf{u}_{B}$, and assume an exponential time evolution, $e^{\lambda t}$, of the velocity. These operations and assumptions result in an independent generalized eigenvalue problem, for each wave-number pair $(l, n)$.

$$
\left(\lambda \mathbf{A}_{l n m^{\prime} m}-\mathbf{B}_{l n m^{\prime} m}(R e)\right) \mathbf{a}_{l n m}=0 .
$$

We compute the eigenvalues, $\lambda$, of (4.1), at the reference Reynolds number $R e_{c l}=9600$ 
and $(l, n)=(1,1)$, and the least stable 20 eigenvalues at $R e_{c l}=30000$, and $(l, n)=$ $(0,0: 1)$ for a pipe length of $L=2 \pi$. The results are presented in Table 4.1 and 4.2 . for various values for the number of selected radial modes (M). An agreement up to 10 digits is obtained with those computed and cited in [13, [81. It is also possible to observe the convergence behaviour from Table 4.1, as the resolution (which depends on the number of radial modes, M, used) is increased. The findings suggest spectral accuracy is achieved, as expected.

Table 4.1: Leading eigenvalue of (4.1) corresponding to $R e_{c l}=9600$, and $(\mathrm{l}=1, \mathrm{n}=1)$

\begin{tabular}{|l|c|}
\hline $\mathbf{M}$ & $\lambda_{\mathbf{1}}$ \\
\hline 20 & $-0.022998556376+0.950174903582 \mathrm{i}$ \\
\hline 30 & $-0.023170716674+0.950481315400 \mathrm{i}$ \\
\hline 40 & $-0.023170795763+0.950481396669 \mathrm{i}$ \\
\hline 50 & $-0.023170795764+0.950481396669 \mathrm{i}$ \\
\hline $50[82]$ & $-0.023170795764+0.950481396670 \mathrm{i}$ \\
\hline
\end{tabular}

In addition Table 4.1 also represents the convergence of the eigenvalue to the reference value with the number of selected radial modes $\mathrm{M}$, even at $\mathrm{M}=30$ the solution is accurate for singel precision calculations.

Table 4.2: The least stable 20 eigenvalues for $R e_{c}=3000 \mathrm{l}=1, \mathrm{n}=0,1$

\begin{tabular}{|l|l|}
\hline$-0.051973111282644+0.948360222051104$ & $-0.041275644693407+0.911465567623395$ \\
\hline$-0.051973123205372+0.948360198487025$ & $-0.061619018004190+0.370935092696298$ \\
\hline$-0.103612364040202+0.896719200868016$ & $-0.088346025188592+9.58205542990992$ \\
\hline$-0.103612889227902+0.896720444100115$ & $-0.088870156636980+8.54788817414939$ \\
\hline$-0.112217160388022+0.412396334210064$ & $-0.116877153586895+2.16803862997431$ \\
\hline$-0.121310028245816+0.218435814727458$ & $-0.137490337053408+7.99699469657846$ \\
\hline$-0.155220165293265+0.845071799711795$ & $-0.144346144867780+9.10037309551185$ \\
\hline$-0.155252667199022+0.845080668126344$ & $-0.186432986148937+7.45304357825299$ \\
\hline$-0.200463047767108+0.376242360026110$ & $-0.195839466536939+5.49311582651879$ \\
\hline$-0.206476811414885+0.793784129833576$ & $-0.198646109004200+8.60749463479149$ \\
\hline$-0.206892849012486+0.793440799040388$ & $-0.204955511398794+3.76431414665149$ \\
\hline$-0.227465621406848+0.626296998166060$ & $-0.234333469326204+6.93462325172212$ \\
\hline$-0.257315715842993+0.502037310430882$ & $-0.251809086876346+5.02642513256198$ \\
\hline$-0.258508466819554+0.741757503077691$ & $-0.252123495622001+8.10844875959245$ \\
\hline$-0.258880615514801+0.747046472275328$ & $-0.270458581648370+3.25130263224953$ \\
\hline$-0.297526502769712+0.347392291849359$ & $-0.289647206916430+6.55064338952368$ \\
\hline$-0.301051765118837+0.610862593988543$ & $-0.305126503954746+7.60702408738928$ \\
\hline$-0.308166308458014+0.692606209723818$ & $-0.309010453617850+6.07934472120832$ \\
\hline$-0.324318059574355+0.710304305793957$ & $-0.344790480797114+5.87438024854394$ \\
\hline$-0.370532966168264+0.674579596375438$ & $-0.359140146606135+7.11236702877725$ \\
\hline
\end{tabular}


It is also possible to plot the associated eigenmodes ans the streamline associated with the computed eigenvalues. This can be facilitated by defining a spiral transformation [82,

$$
\frac{d z}{d \theta}=-\frac{n}{l}
$$

where $n, l$ are the azimuthal and axial wavenumbers respectively, under which the solenoidal variables, such as velocity, are invariant. Following [82], one can define a new coordinates, also called as spiral coordinate,

$$
\zeta=n \theta+l z
$$

After some manipulation (for details consult [82]), one can construct $\Xi$ such that particle movement is constrained to $\Xi=$ constantsurfaces, using,

$$
\begin{aligned}
\partial_{\zeta} \xi & =-r u \\
\partial_{r} \xi & =n v+r l w \\
\mathbf{u} & =2 \mathcal{R} e^{l z+n \theta} \sum_{0}^{M} a_{m} v_{m}
\end{aligned}
$$

Case 1: $l=1, n=0$, The associated stream function is shown in Figure 4.1. The corresponding vector field is depicted in Figure 4.2.

Case 2: $\mathrm{l}=0, \mathrm{n}=1$, the associated stream function is shown in Figure 4.3 . The corresponding vector field is depicted in Figure 4.4.

Case 3: $\mathrm{l}=1, \mathrm{n}=1$, the associated stream function is shown in Figure 4.5. The corresponding vector field is depicted in Figure 4.6.

\subsection{Transient Growth of Perturbations}

In this section, results of the numerical approach for the study of transient growth of perturbations are presented. To asses the growth of perturbations, we introduce the define the energy of a velocity field, $u$ as,

$$
\epsilon=\frac{1}{2}(u, u)
$$




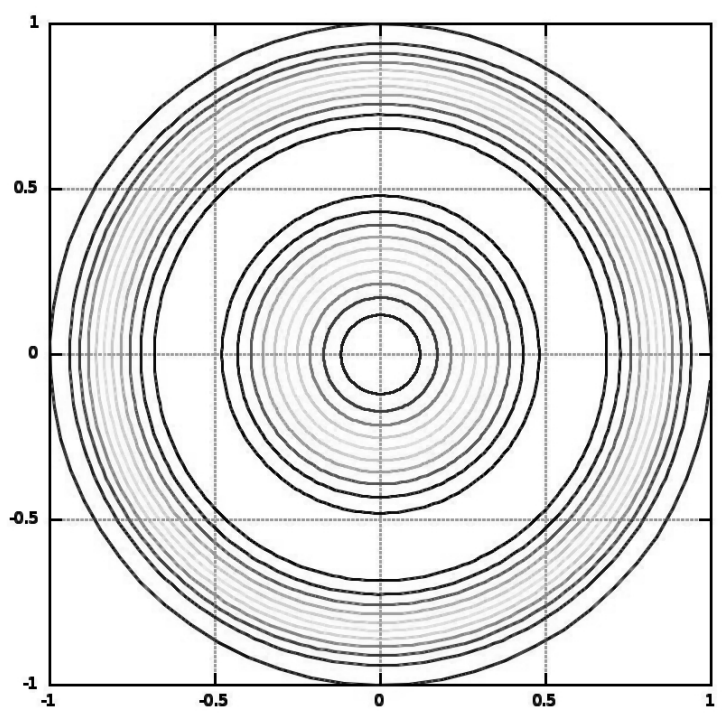

Figure 4.1: Stream function, $\mathrm{l}=1, \mathrm{n}=0, \lambda=-0.0519731113+0.9483602221 i$

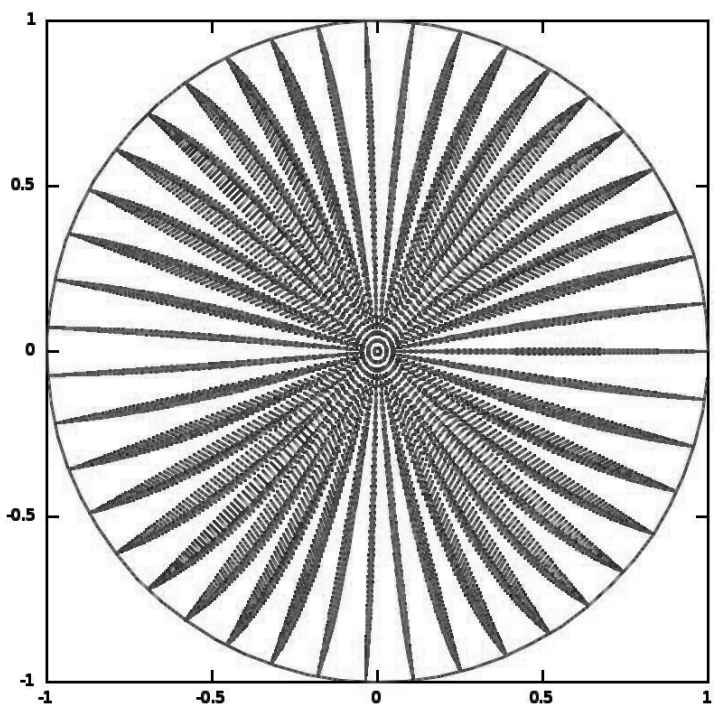

Figure 4.2: Corresponding vector field, $\mathrm{l}=1, \mathrm{n}=0, \lambda=-0.0519731113+0.9483602221 i$ 


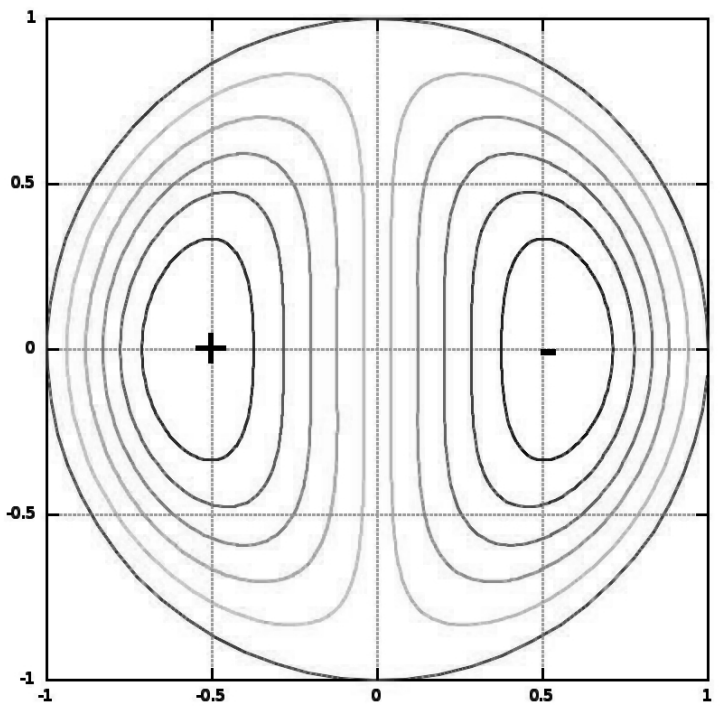

Figure 4.3: Stream function, $\mathrm{l}=0, \mathrm{n}=1, \lambda=-0.0087915391$

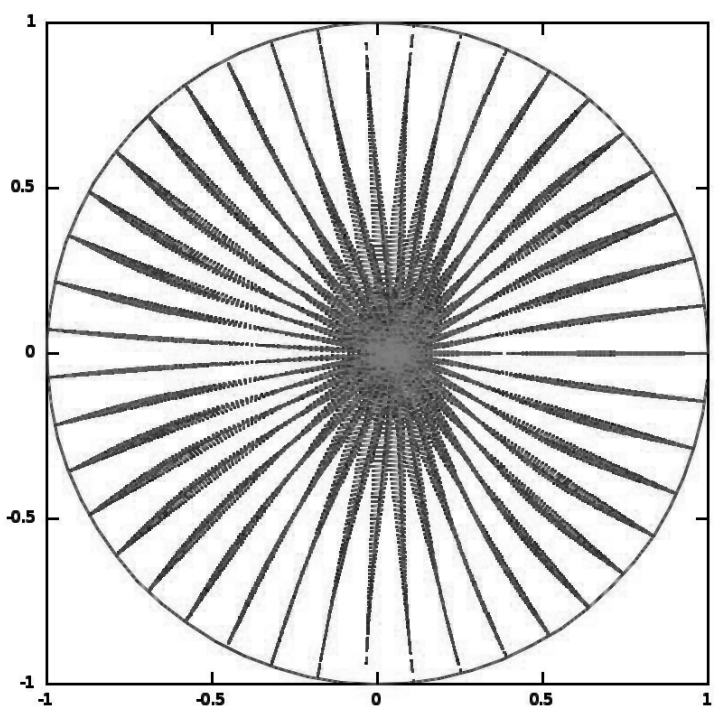

Figure 4.4: Corresponding vector field, $\mathrm{l}=0, \mathrm{n}=1, \lambda=-0.0087915391$ 


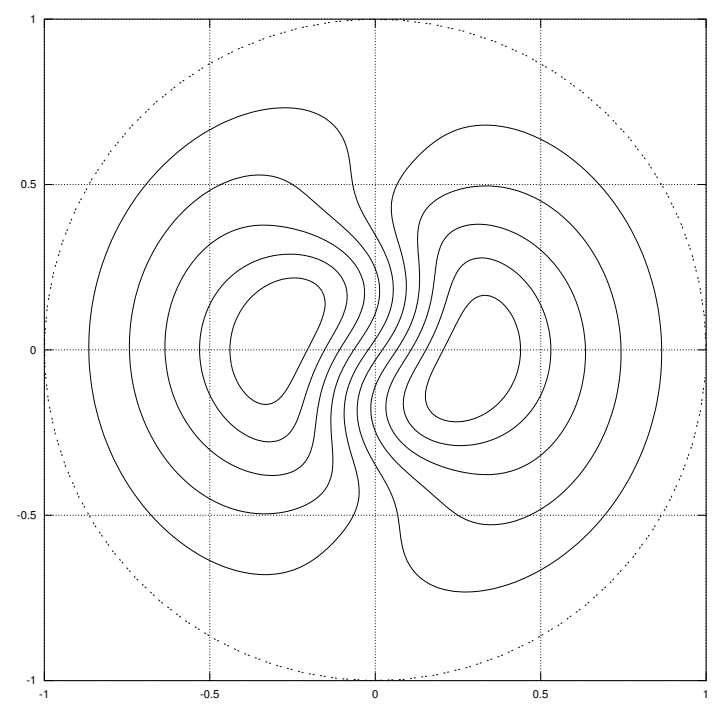

Figure 4.5: Stream function, $\mathrm{l}=1, \mathrm{n}=1, \lambda=-4.1275644693 e-02+9.1146556762 e-01 i$

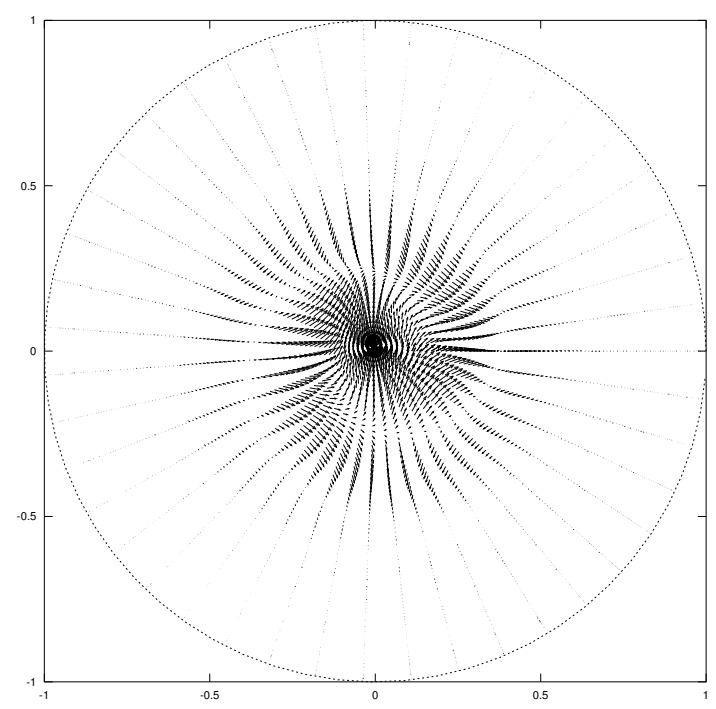

Figure 4.6: Corresponding vector field, $\mathrm{l}=1, \mathrm{n}=1, \lambda=-4.1275644693 e-02+$ $9.1146556762 e-01 i$ 


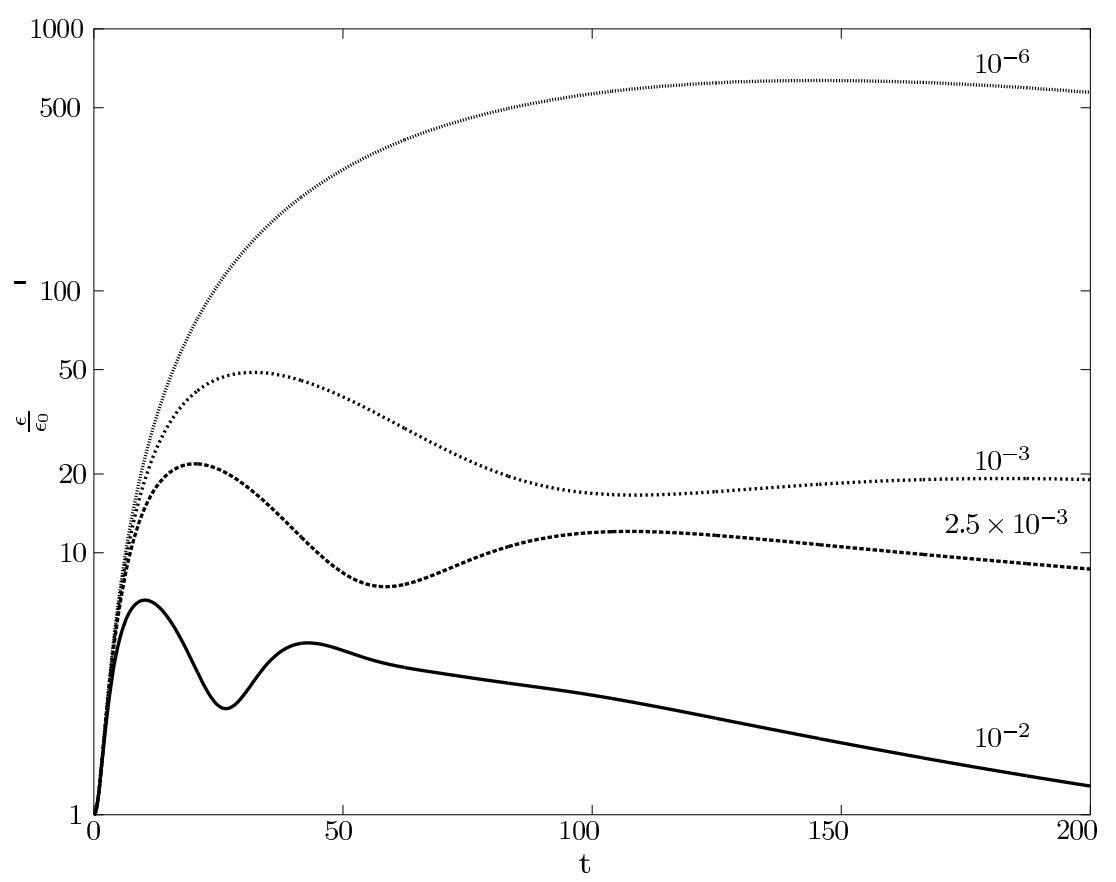

Figure 4.7: Time evolution of various $2 \mathrm{D}$ perturbations at $\mathrm{Re}_{\mathrm{cl}}=3000$ where energy $\epsilon$ is measured relative to the initial disturbance energy $\epsilon_{0}$.

where the paranthesis signify the inner product defined in (3.5). First, the growth factors $\frac{\epsilon}{\epsilon_{0}}$ (instantaneous energy divided by the initial energy) associated with an initial two-dimensional disturbance

$$
a_{l n m}^{0}=\left\{\begin{array}{lll}
A_{0} & \text { for } & l=0, n= \pm 1, m=0 \\
0 & & \text { otherwise }
\end{array}\right.
$$

are presented for centerline Reynolds number, $R e_{c l}$ of 3000 with 8 modes in the radial direction $(M=8), 19$ in the azimuthal direction (number of strictly positive azimuthal Fourier modes, $N=9$ ), and finally 3 in the axial direction (number of strictly positive axial Fourier modes, $L=1$ ), with $\Delta t=5 \cdot 10^{-2}$ in Figure 4.7. For $\epsilon_{0}=10^{-6}$, a maximum amplification factor of $\sim 630$ is obtained at $t \sim 146$, while for $\epsilon_{0}=10^{-5}$, it is $\sim 600$ at $t \sim 142$ in accordance with [87]. The corresponding streak formation for $\operatorname{Re}_{\mathrm{cl}}=3000$ with $\epsilon_{0}=10^{-2}$ is shown in Figure 4.8 and it is in qualitative aggrement with [77, 87].

As another numerical experiment, the current numerical approach is also tested regarding the effect of the spanwise wall oscillations on the growth of perturbations. This has been the subect of various drag reduction studies in literature [37, 42]. For this purpose, the scaling based on the friction units is used. Figure 4.7 presents the 

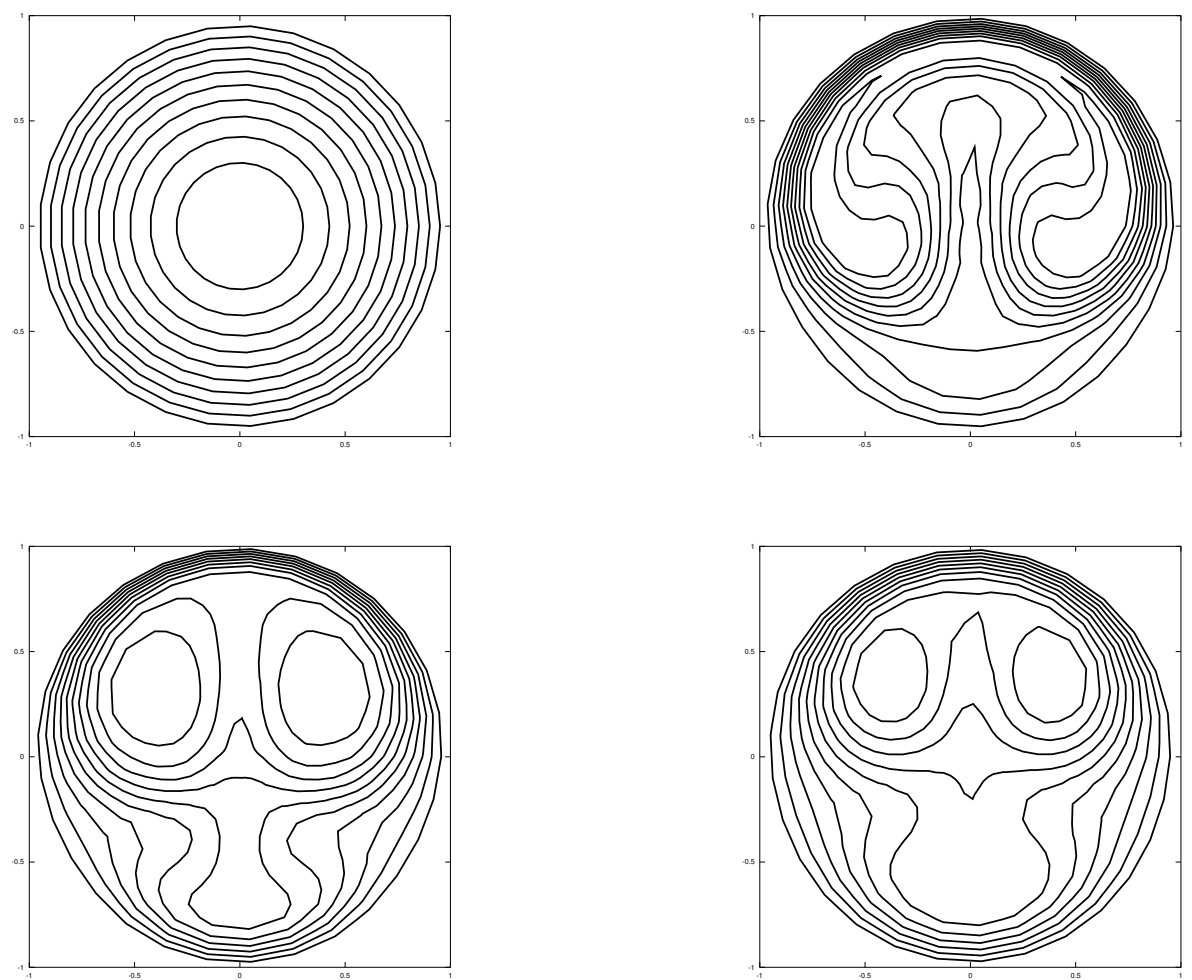

Figure 4.8: Streak formation for $\mathrm{Re}_{\mathrm{cl}}=3000$ with $\epsilon_{0}=10^{-2}$ in the axial flow at $t=0,17,75,150$. 
growth of perturbation (4.3) of $\epsilon_{0}=10^{-2}$ and Figure 4.9 shows the corresponding change in the mean axial velocity for the spanwise wall oscillations of the form $\left.v_{\theta}\right|_{r=1}=A^{+} \sin \left(\Omega t^{+}\right)$. These results were obtained for the friction Reynolds number $100\left(R e_{\tau}=\frac{u_{\tau} R}{\nu}\right)$ and for the frequency of oscillations $\Omega=2 \pi / 50$. The time in the plot is in wall units $\left(t^{+}=\frac{u_{\tau}^{2} t}{\nu}\right)$. The preliminary computations show that the imposition of the spanwise wall oscillations enhances the decay of perturbations and the convergence back to the parabolic profile in accordance with the literature. These are encouraging to further our studies on the the effects of the spanwise wall oscillations using the current numerical approach.

The main advantage of using solenoidal bases in incompressible fluid flow problems is that the solution is strictly solenoidal (i.e. divergence-free) throughout the solution domain. This eliminates the possible effects of the errors associated with poor resolution of the continuity equation. In addition, the elimination of the pressure due to the projection onto the dual solenoidal basis simplifies the algorithm by removing the need for a pressure solver. In contrast to the popular solenoidal Karhunen-Loeve (POD) bases, no separate simulation stage is required in the generation of the current bases. In regards to the energy optimality in POD bases, on the other hand, the POD bases are no longer optimal when used for off-reference parameter values while the current bases are parameter-independent. The main disadvantage of the current analytic solenoidal bases is that they are generally limited to simple geometries. However, there are domain decomposition approaches (see [34]) as a possible remedy. 


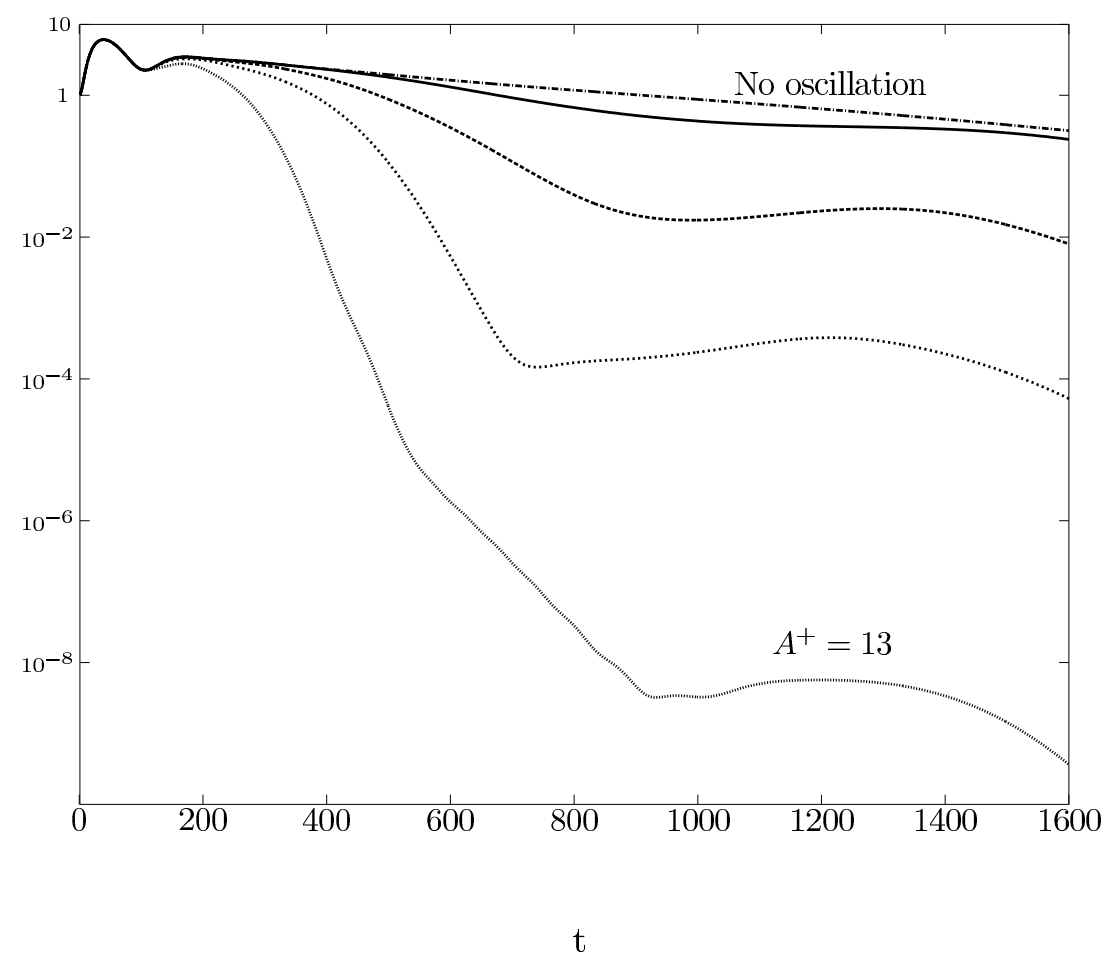

Figure 4.9: Decay of 2D perturbation energy $\epsilon_{0}$ versus $t^{+}$for various oscillation amplitudes $A^{+}=0,1,2.5,5,13$.

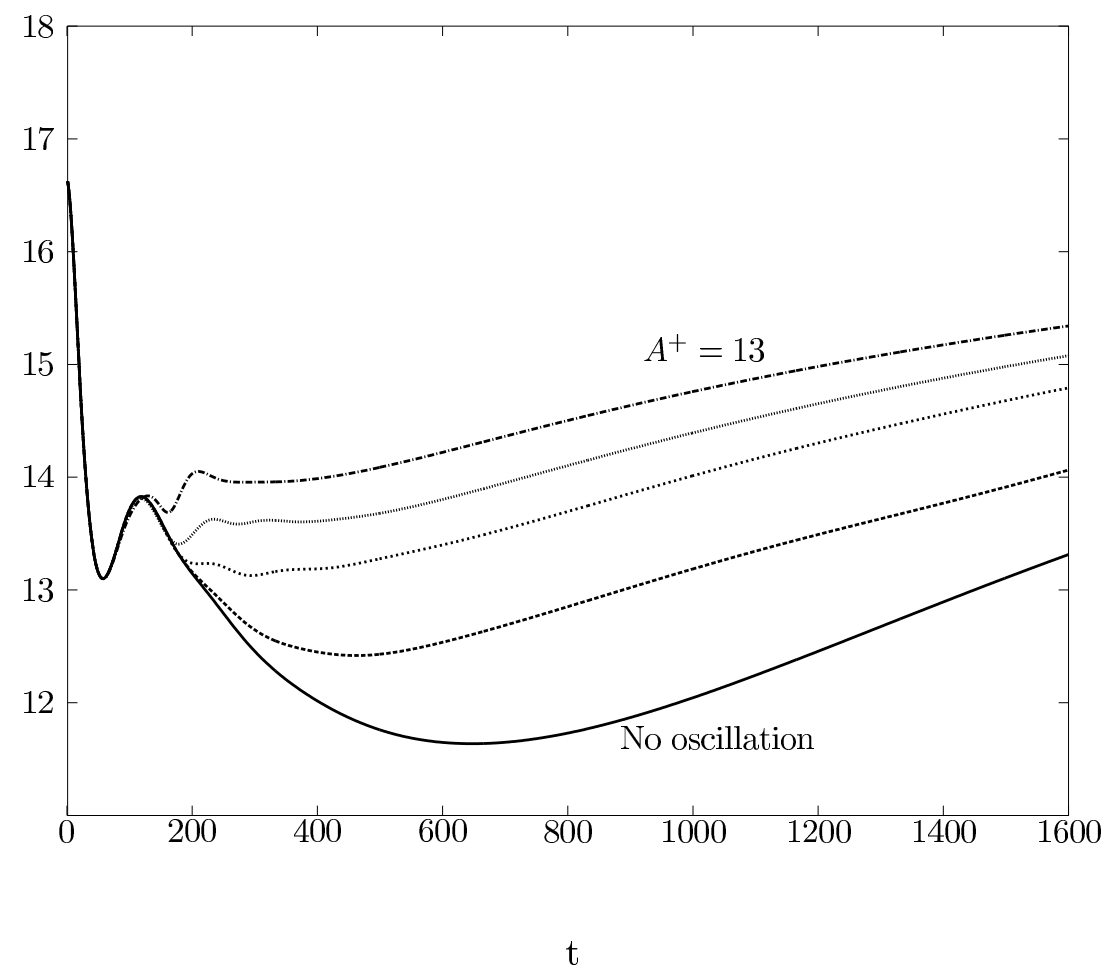

Figure 4.10: Mean axial velocity for 2D perturbation versus $t^{+}$for various oscillation amplitudes $A^{+}=0,1,2.5,5,13$. 


\subsection{Benchmark cases for turbulent pipe flow at low Reynolds number}

This section presents benchmark cases for the numerical code, for both constant pressure and constant mass flux driven flows. The main issue in DNS of turbulence in pipe flow, is ironically the triggering of turbulene [87, 30, 88, 89]. One of the main approaches is the use of a formarly obtained turbulent flow field. Having no prior data in this study, however, specially constructed initial conditions has to be used. It is important to note that, as one might be led to believe, starting from random initial conditions will almost always fail [30, as they will unnaturally excite the higher modes, and in turn create excess viscosity. It was previously shown that the suitable initial conditions for triggering turbulence are spatially localized [87, 89, 14. In this study the widely used Zikanov modes [87] are used as initial conditions. One other point is that, it is preferrable to start the simulation at a higher Reynolds number than intended, so as to suppress the viscous effects while a turbulent flow field is achieved, and then gradually decrease (relax) the Reynolds number until the desired simulation parameter is achieved. For the constant mass flux case, a turbulent flow field from the constant pressure case was used as a starting point, with proper scaling, and the same Reynolds number relaxation was performed. With the correct choice of initial conditions and Reynolds number relaxation, transition to turbulence was abrupt, as commonly reported [88]. The initial conditions used for the constant pressure case are plotted in 4.11(a), 4.11(b), 4.11(c) at the pipe inlet, where they are localized.

\subsubsection{Constant Pressure Gradient Case}

After completing the validation for the linear case, we compute the turbulence statistics at $R e_{\tau}=180$. The computations were performed for a pipe with a length of 10 radii (i.e $L=10$ ). The results are presented in Table 4.3 and in Figures 4.124 .18 in comparison with the DNS and experimental results presented in [30], and the numerical results from 31. Fukagata et al. 31] use a highly energy conserving scheme based on a finite difference method, with a resolution (radial $\times$ azimuthal $\times$ axial) of $96 \times 128 \times 256$, whereas Eggels et al. [30] use a finite volume discretization with the same resolution. In our method we do not impose any explicit energy conserving constraint on the system and use a resolution of $53 \times 127 \times 227$. Our computations 


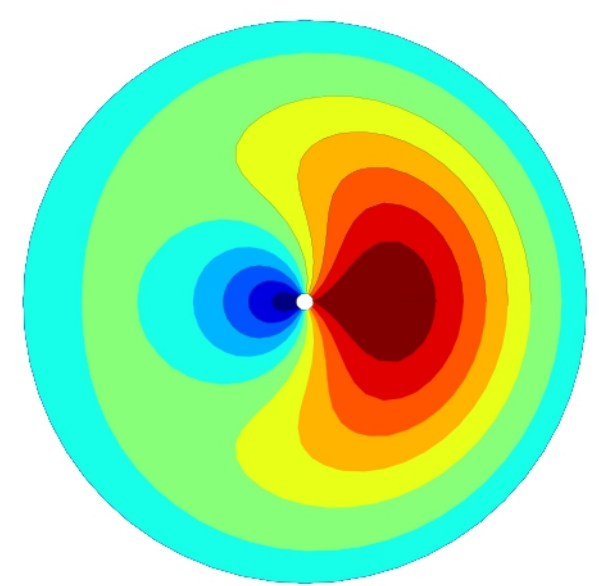

(a) Initial conditions, contours of radial velocity

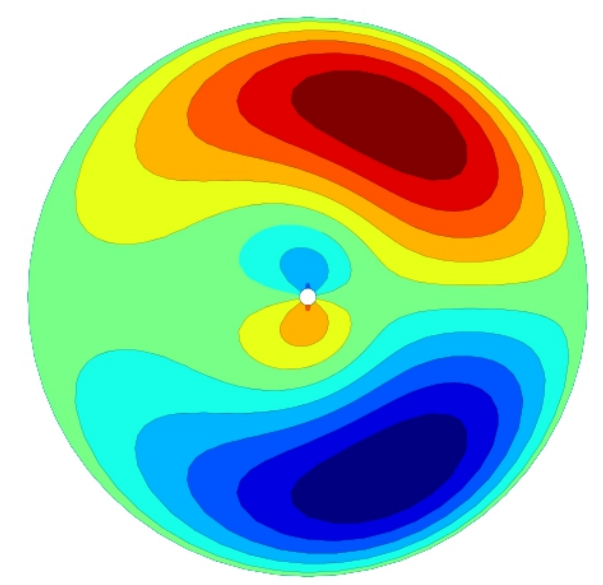

(b) Initial conditions, contours of azimuthal velocity

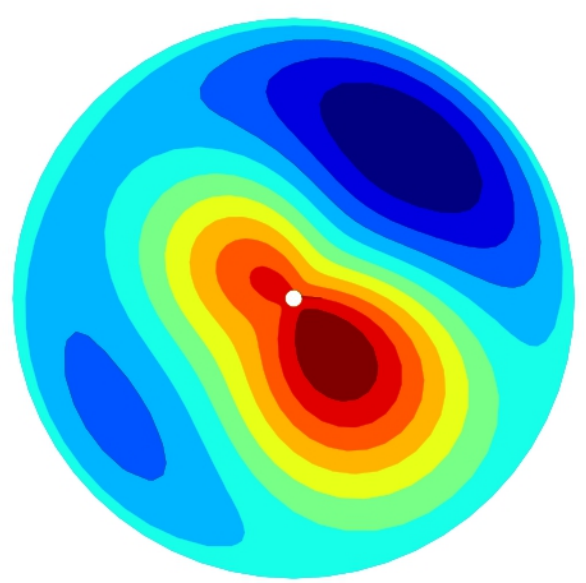

(c) Initial conditions, contours of axial velocity

Figure 4.11: Axial averages of initial conditions for the simulations 
are fully de-aliased in the periodic directions using the $3 / 2$ rule and, a time step of $2.5 \times 10^{-4}$ non-dimensional time units. The statistics are computed using 100 flow fields equispaced in time with a spacing of 0.4 non-dimensional time units after discarding the initial transients, amounting to 100000 timesteps, and 40 frictional time units. It is worthwhile to note that even though we use a coarser grid, we have seven grid points within the viscous sublayer whereas [30] have only three. Thus, our grid is denser near the walls in comparison. It is widely known that spectral methods have superior energy conserving properties [90, 83, 80, 91] when compared to finite difference methods. In addition, Galerkin type projections are known to be stable in the energy sense [91].

In regards to turbulence statistics, in Figures $(4.12,4.18)$, it can observed that, our method can predict the turbulence statistics with a relative error in the order of $3 \%$. The main difference with the reference calculations is that, our code predicts rms velocity fluctuations closer to the experimental results [30] in the near wall region (Figure 4.17) and seems to match the peak experimental Reynolds stress magnitude closely (Figure 4.18). We believe that this increased predictive power at a lower resolution comes from the combination of accuracy of spectral methods, inherent satisfaction of the continuity equation by solenoidal bases expansion and the fact that we have a denser grid composed of Gauss-Legendre points in the near wall region. However, when comparing the results for the core region (near the pipe centerline), our method under-predicts the rms fluctuations of the azimuthal velocity by a fair margin. In addition, the computed mean axial velocity is lower than suggested by experimental results. This however, is expected as our grid is relatively coarse in the core region. In the light of these observations it is safe to say that, at the resolutions employed, our method provides increased accuracy in the wall region at the expense of predictive power in the center region.

In addition to friction scaling, a simulation keeping the bulk velocity constant is also performed, at Re $=5200$. This Reynolds number roughly corresponds to our earlier simulation at $R e_{\tau}=180$. In keeping the bulk velocity constant, at a value of 0.5 after non-dimensionalization, an adjustable pressure gradient is used in contrast to a constant pressure gradient used in the friction scaling case. For this case the simulation has been carried out for 600 non-dimensional time units (in bulk scaling) 
Table 4.3: Turbulence statistics for pipe flow in the cases of (1) constant pressure gradient, (2) constant mass flux.

\begin{tabular}{l|ccccc} 
& $\begin{array}{c}\text { Present } \\
(1)\end{array}$ & $\begin{array}{c}\text { Present } \\
(2)\end{array}$ & $\begin{array}{c}\text { Quadrio et al. } \\
(\mathrm{DNS})(2)[\underline{40}]\end{array}$ & $\begin{array}{c}\text { Eggels et al. } \\
(\mathrm{DNS})(1)[\underline{30}]\end{array}$ & $\begin{array}{c}\text { Eggels et al. } \\
(\mathrm{PIV})(1)[\underline{30}]\end{array}$ \\
\hline$U_{c l} / U_{B}$ & 1.32 & 1.32 & 1.31 & 1.31 & 1.30 \\
$U_{B} / u_{\tau}$ & 14.58 & 14.24 & 14.24 & 14.73 & 14.88 \\
$U_{c l} / u_{\tau}$ & 19.27 & 18.79 & 18.63 & 19.31 & 19.38 \\
$c_{f}$ & 0.00941 & 0.0098 & 0.00963 & 0.00922 & 0.00903 \\
$\operatorname{Re}_{c l}$ & 6864 & 6468 & 6400 & 6950 & 7100 \\
$\operatorname{Re}$ & 5200 & 4900 & 4900 & 5300 & 5450 \\
$\operatorname{Re}_{\tau}$ & 180 & 173 & 172 & 180 & 183
\end{tabular}

Table 4.4: Maximum allowable time step, in units of $2 t U_{B} / R$

\begin{tabular}{|c|c|c|}
\hline $\mathrm{N}$ & $\Delta t$, clustered grid & $\Delta t$, non-clustered grid \\
\hline 25 & 0.00282 & 0.0156 \\
\hline 50 & 0.000775 & 0.00625 \\
\hline 100 & 0.0000477 & 0.00196 \\
\hline
\end{tabular}

after discarding the initial transients as in [40], a total of 150 flow fields equidistant in time were employed in calculation of the statistics. The findings are summarized in Table 4.3 along with the earlier studies ([30], [40]).

During our computations, the average CFL number computed to be equal to 0.4 , as defined by,

$$
\mathrm{CFL}=\max \left(\frac{u_{r m s} \Delta t}{\Delta r}+\frac{v_{r m s} \Delta t}{r \Delta \theta}+\frac{w_{r m s} \Delta t}{\Delta z}\right),
$$

over the grid, where the subscript rms denotes root mean square value of velocity component. As mentioned before, our code uses a non-clustered grid configuration in the radial direction (see Fig.3.1). Table 4.4 gives the maximum allowable time step $(C F L<1)$, as a function of number of radial grid points for both clustered and non clustered cases for the reference velocities computed above, it is evident that the time step restriction is quite severe for a clustered grid configuration. The code, with the resolution stated above, uses approximately $4 \mathrm{~Gb}$ of memory and serial calculation for a single time step takes 2.5 seconds on a $2.8 \mathrm{GHz}$ i7 CPU. 


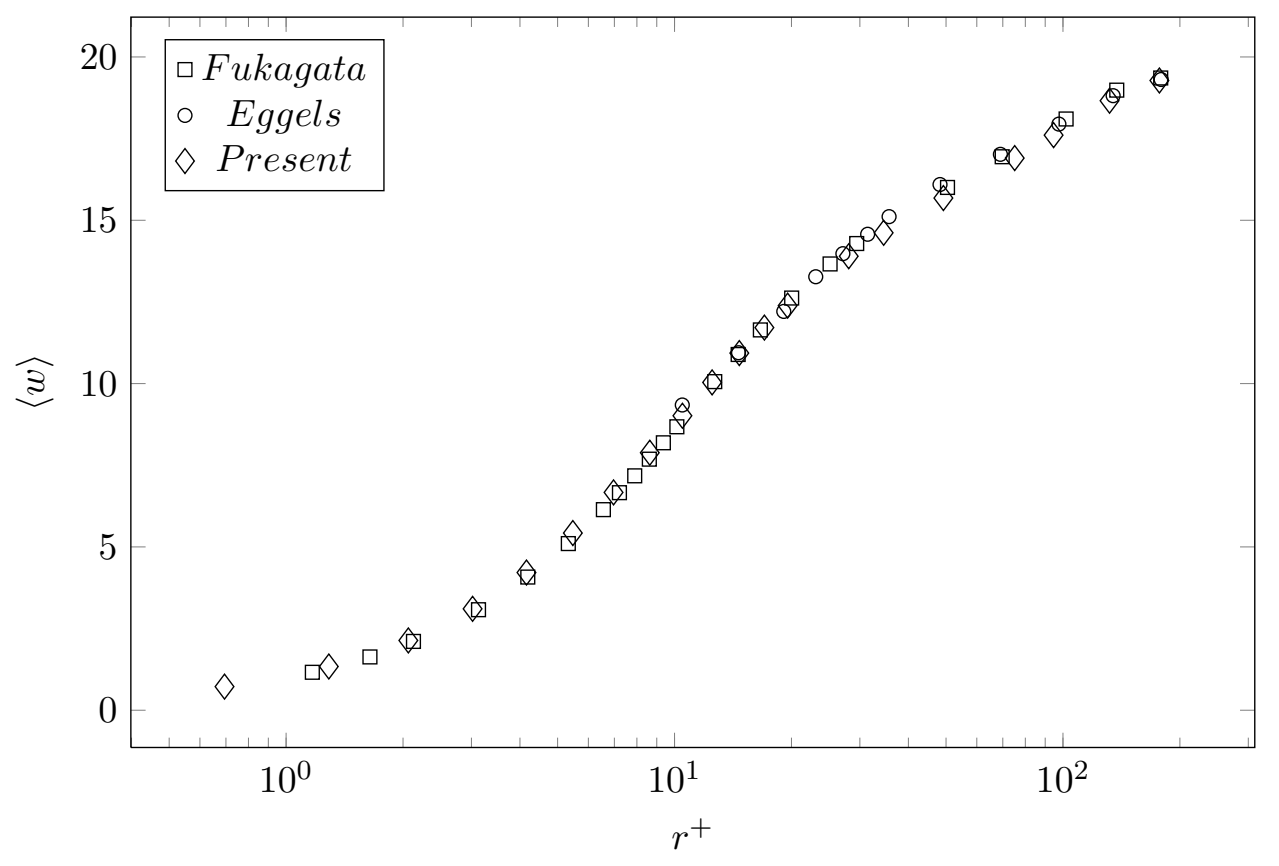

Figure 4.12: Semi-logarithmic plot of mean axial velocity,'o' PIV Eggels et al. [30], Fukagata et al. [31, ' $\diamond$ ' present study where $r^{+}=(1-r) \operatorname{Re}_{\tau}$

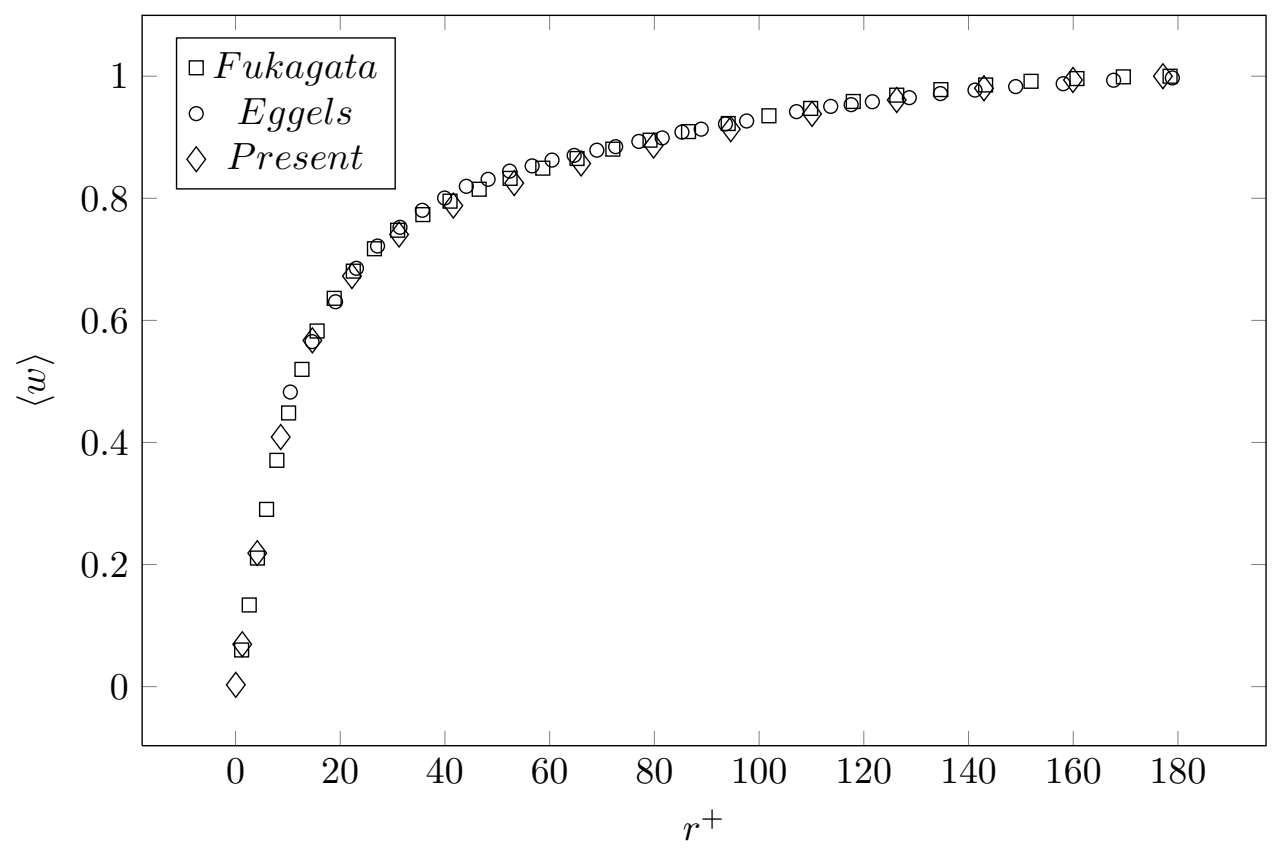

Figure 4.13: Mean axial velocity scaled by mean centerline velocity, 'o' PIV Eggels et al.[30, ' $\square$ ' Fukagata et al. [31], ' $\checkmark$ ' present study. 


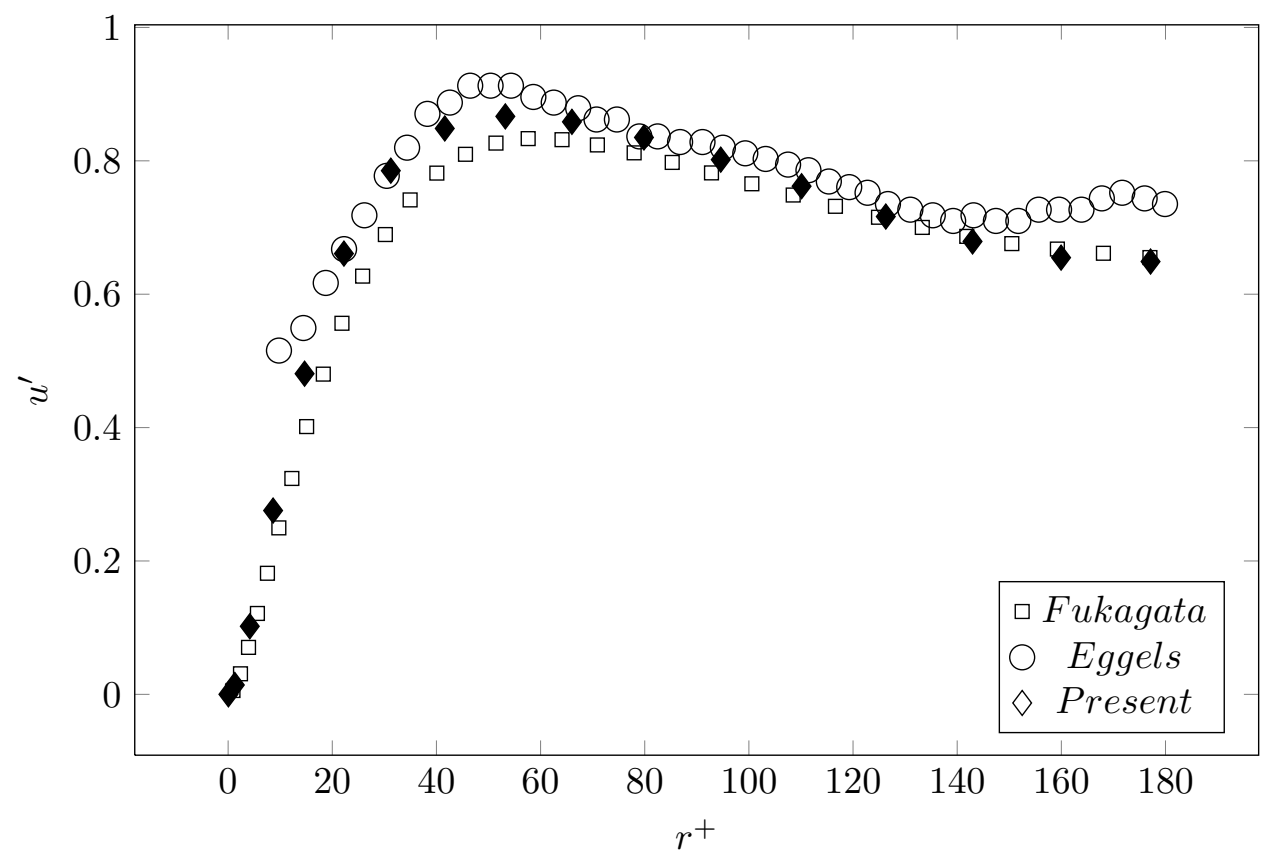

Figure 4.14: RMS velocity fluctuations of u,'o' PIV Eggels et al. [30], ' $\square$ ' Fukagata et al. [31, ' $\diamond$ ' present study.

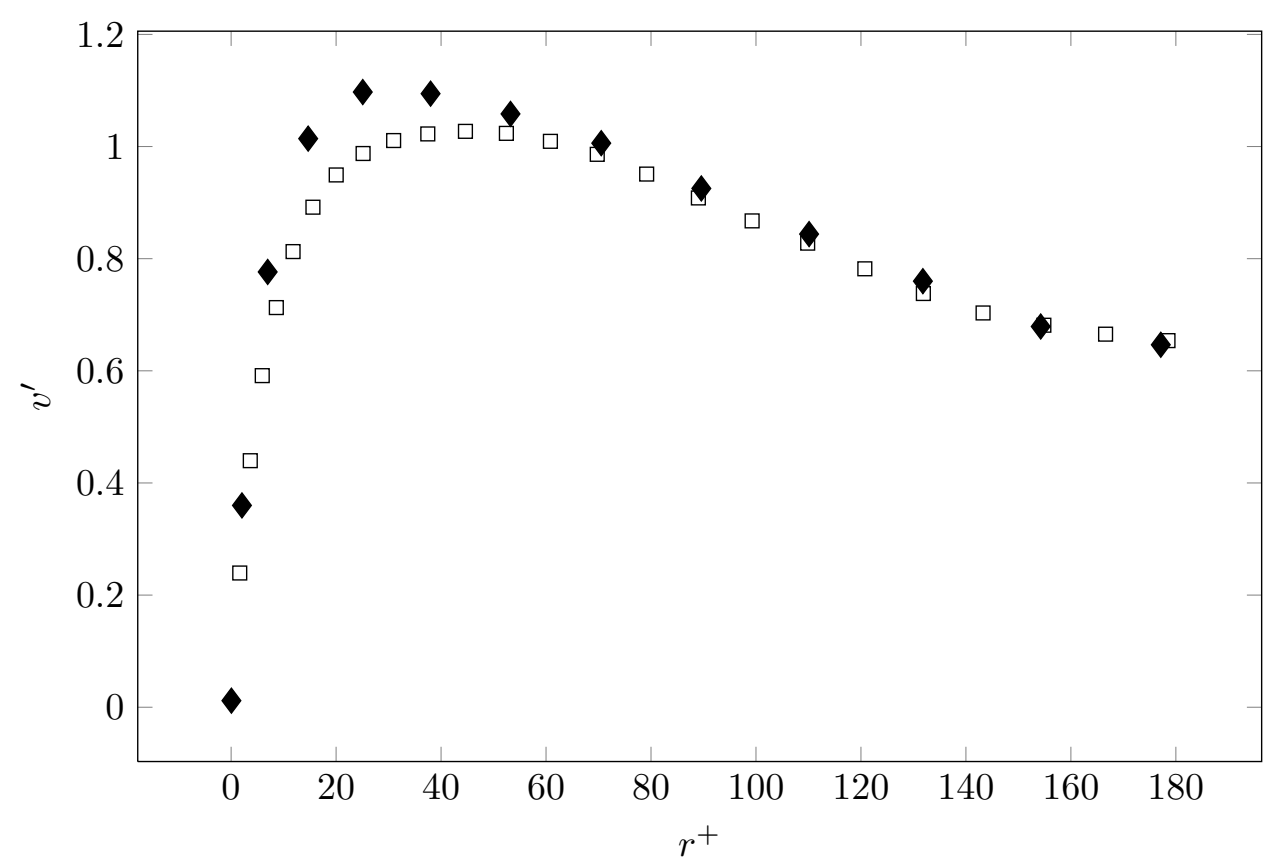

Figure 4.15: RMS velocity fluctuations of v,'o' PIV Eggels et al. [30], ' $\square$ ' Fukagata et al. [31], ' $\diamond$ ' present study. 


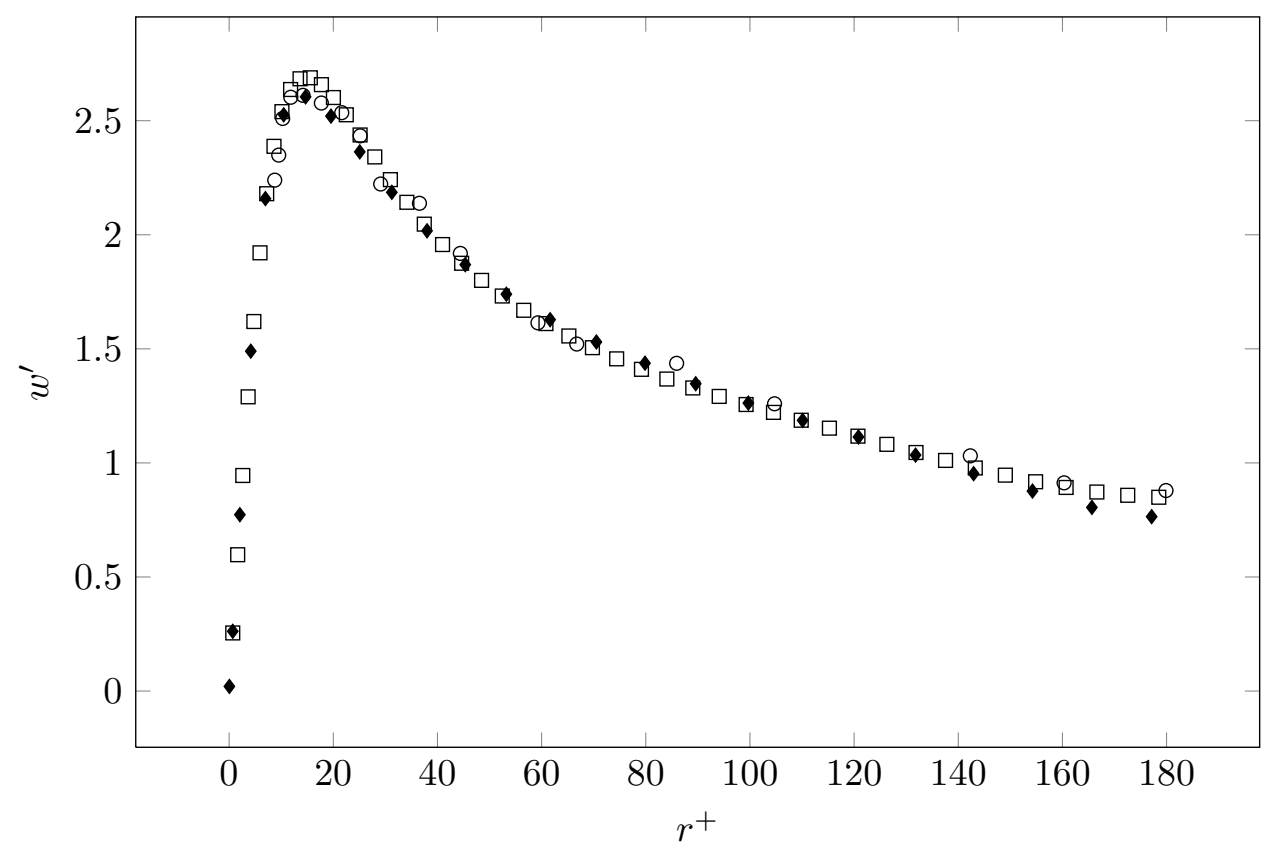

Figure 4.16: RMS velocity fluctuations of w,'o' PIV Eggels et al.[30], ' $\square$ ' Fukagata et al. 31, ' $\diamond$ ' present study.

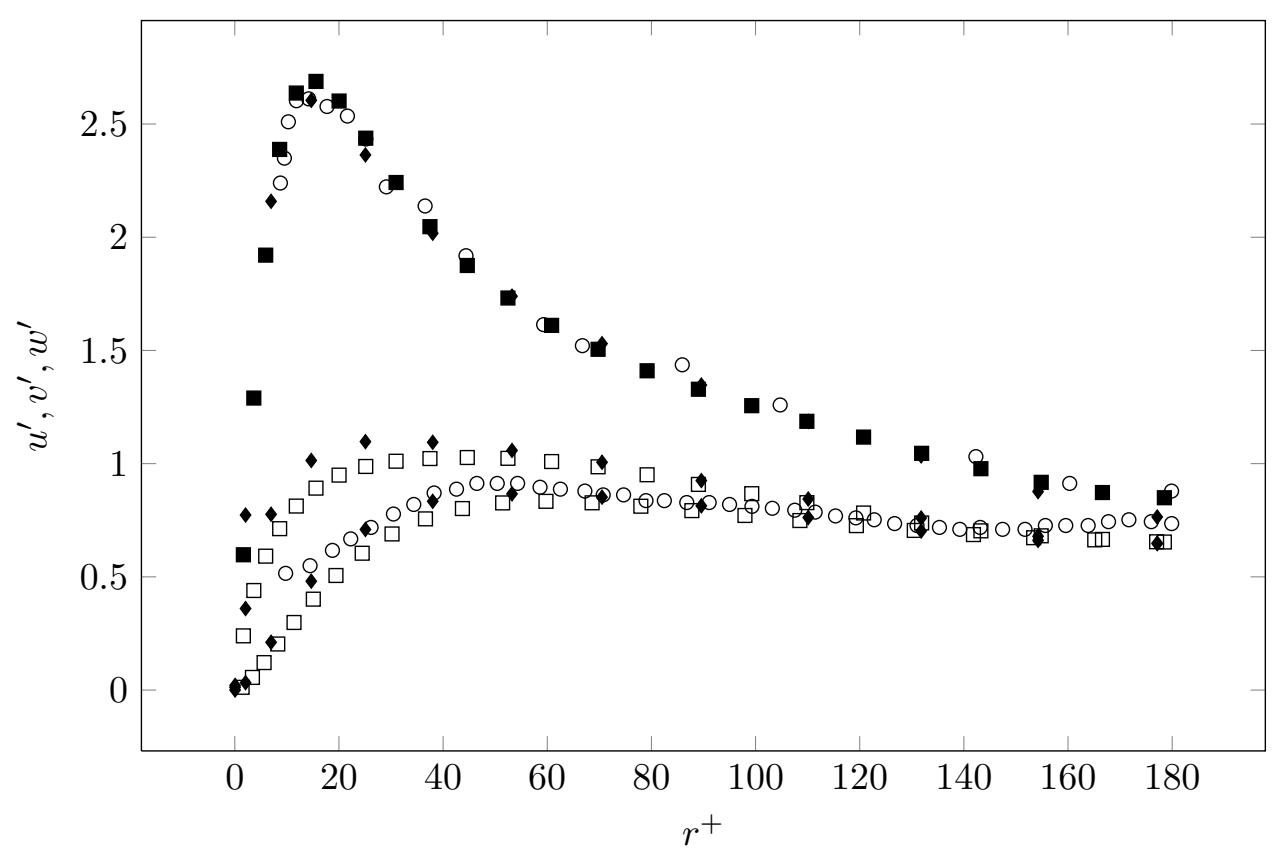

Figure 4.17: RMS velocity fluctuations,'o' PIV Eggels et al.30, ' $\square$ ' Fukagata et al. [31, ' $\diamond$ ' present study. 


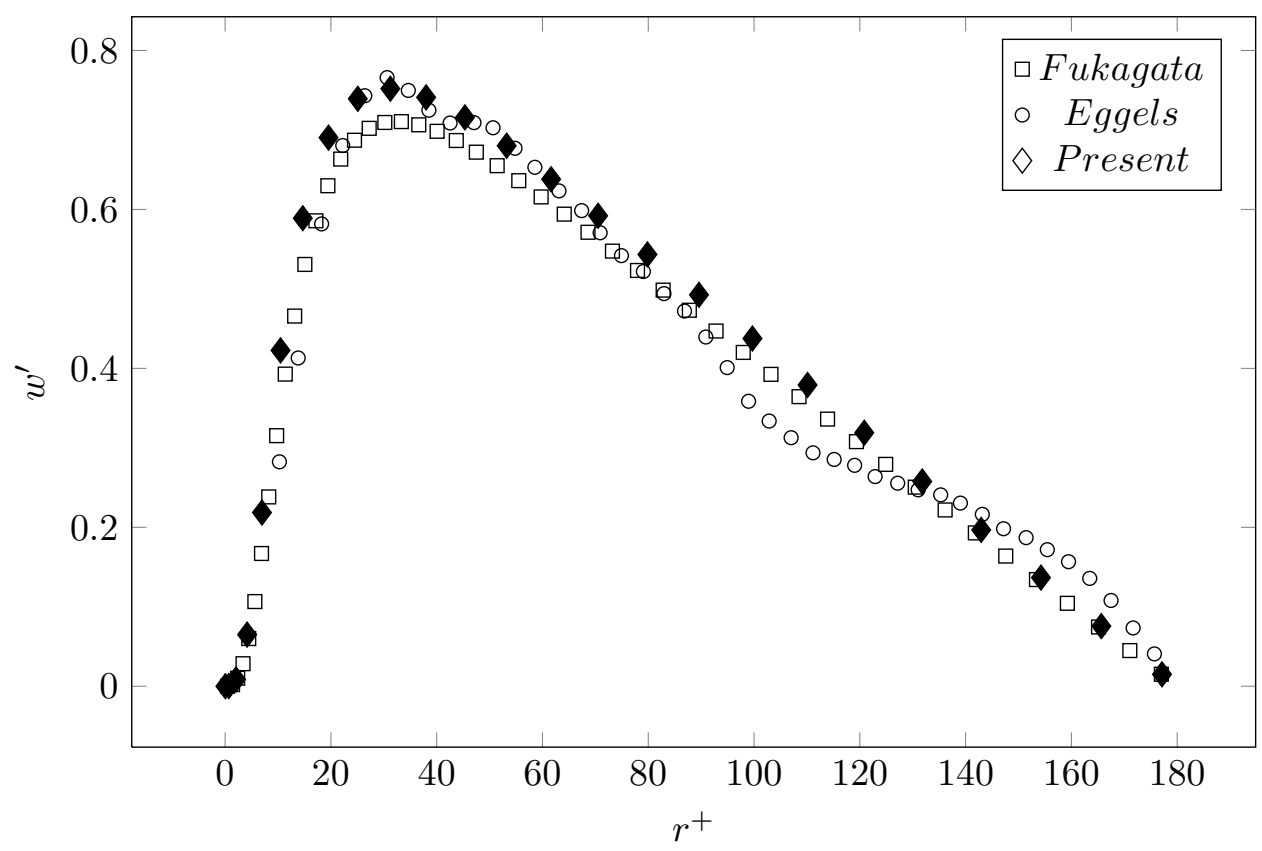

Figure 4.18: Mean Reynolds stress, $\overline{u^{\prime} w^{\prime}}$, 'o' PIV Eggels et al. [30], ' $\square$ ' Fukagata et al. [31, ' $\diamond$ ' present study.

\subsubsection{Constant mass flux}

This section presents simulations using constant mass flux driven flow. For this kind of forcing, as the name implies, the mass flux is kept constant, and is equal to the mass flux in the case of laminar flow. Eventhough a laminar flow and constant mass flux driven turbulent flow have the same flowrate, in the case of turbulent flow the pressure gradient required is about 3 times larger for Reynolds numbers around 5000 .

When the mass flux is kept fixed in a simulation, it is both natural and customary to scale (non-dimensionlize) the NS equations using twice the bulk velocity as the velocity scale, as given in (2.8). The derivation of the constant flux formulation is greatly simplified thanks to the employment of solenoidal basis functions for the expansion of velocity. The detailed derivation of the constant mass flux formulation can be found in Appendix B. The same grid which was used in the constant pressure gradient case was utilized, with a resolution of $53 \times 127 \times 227$, and the time step chosen was $5 \times 10^{-3}$. After the initial transients, the simulation was run for 600 time units for computing the flow statistics, which is equivalent to 120000 time steps. 


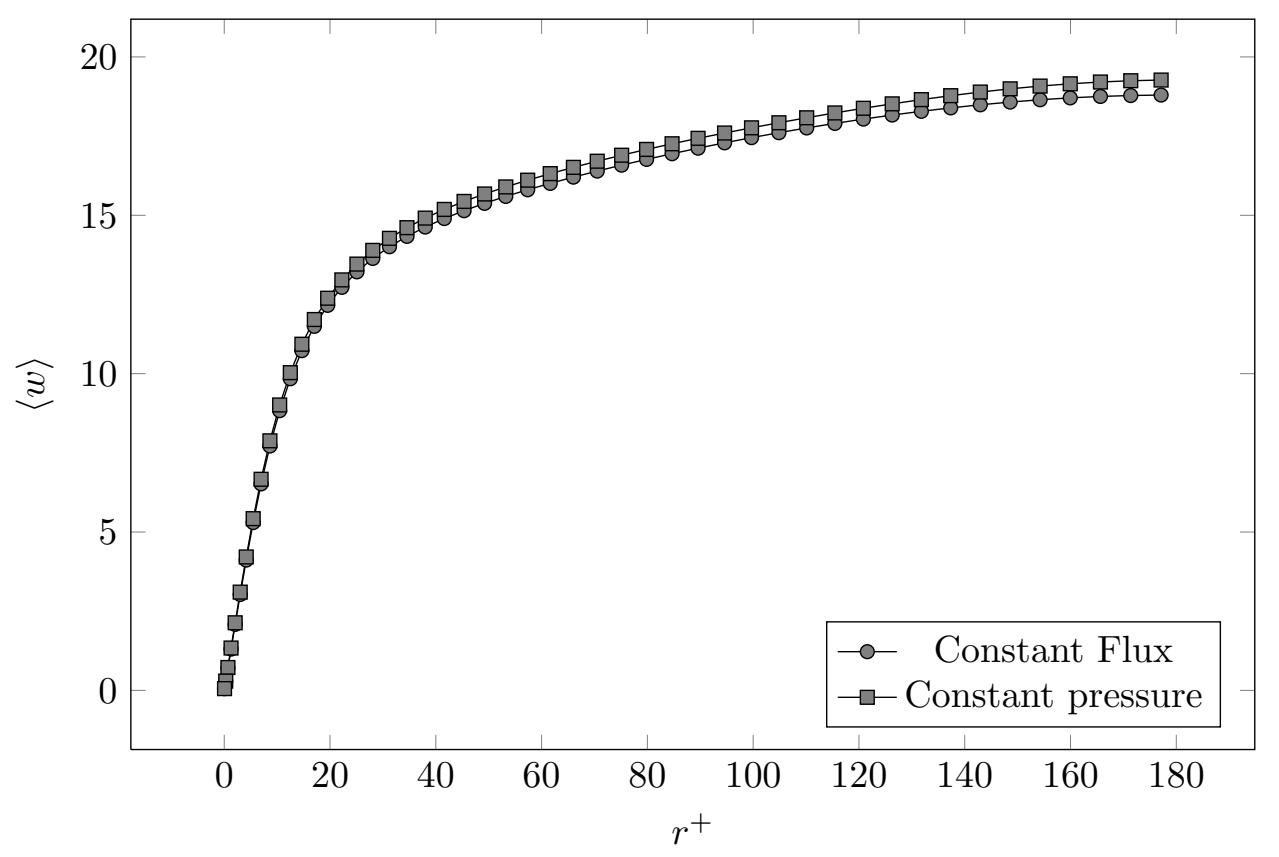

Figure 4.19: Mean streamwise velocity profiles and bulk velocity for constant mass flux case, and the constant pressure gradient case.

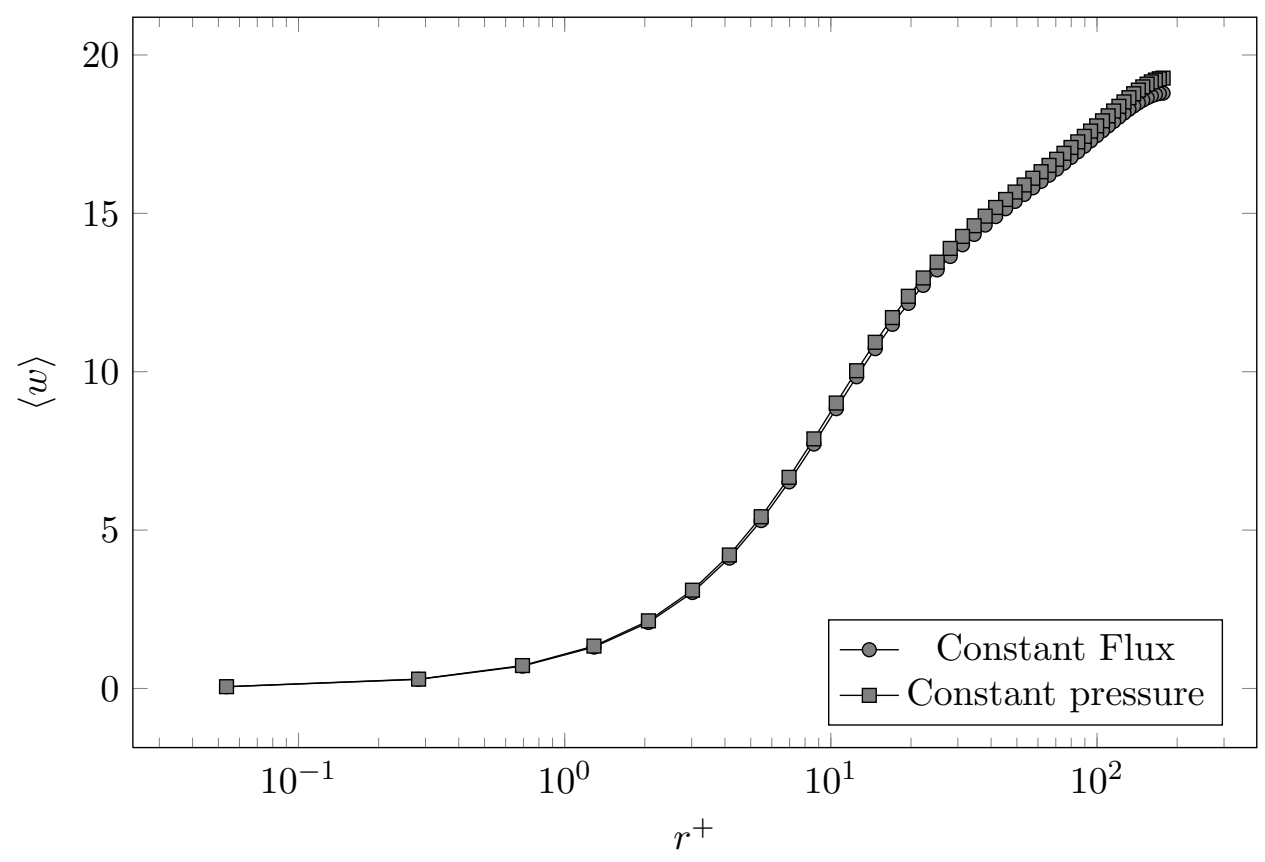

Figure 4.20: Mean streamwise velocity profiles and bulk velocity for constant mass flux case, and the constant pressure gradient case. 


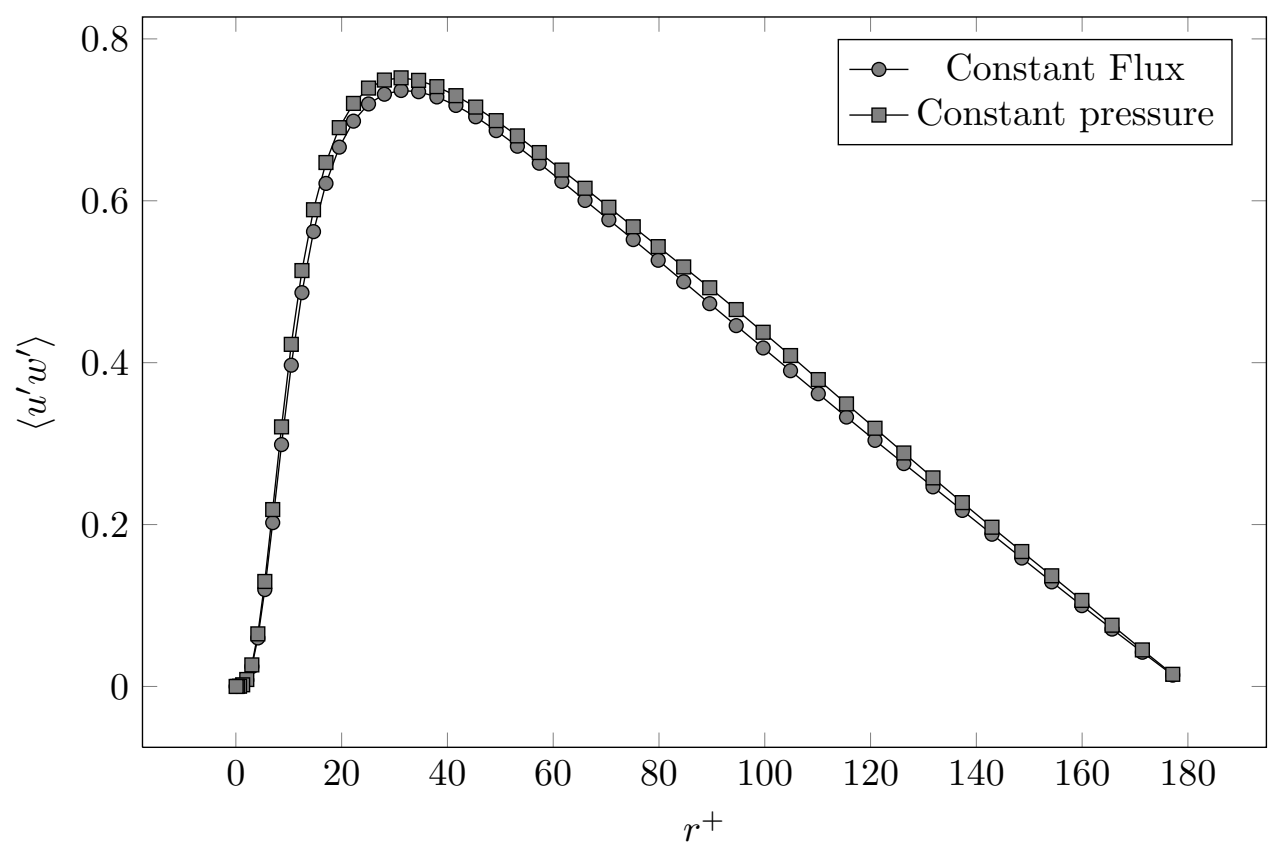

Figure 4.21: Mean Reynolds stress for constant mass flux case, and the constant pressure gradient case.

\subsubsection{Energy conserving property}

In this section, the energy conserving property of the developed numerical method is investigated. In order to investigate the energy conserving properties, one assumes $R e_{\tau} \rightarrow \infty$, which is to say the viscousity tends to zero, with these assumptions, one obtains the Euler equations. For this investigation an instantaneous velocity field from earlier DNS calculations is interpolated on a much coarser grid, the mean velocity is equated to zero, and finally a scaling is applied to render the flow kinetic energy unity, as in [31]. This is followed by the removal of the forcing term and also the dissipative terms (which correspond to the second derivative terms in N-S equations) in the governing equations are dropped. As mentioned previously, these modifications lead to the Euler equations with no forcing and an initial field of zero mean velocity. We simulate this system, follow the change in kinetic energy and note the time it takes for the energy to increase by $1 \%\left(t_{1}\right), 50 \%\left(t_{50}\right)$ and diverge $\left(t_{d i v}\right)$. Our results are summarized in Table 4.5 in comparison to [31]. It is evident that the presented method has very good energy conservation properties. We beleive this is due to the inherent satisfaction of the continuity equation, imposed regularity of the solution at $r=0$ and the efficiency of spectral methods in conserving the energy. 
Table 4.5: Growth of kinetic energy as $R e_{\tau} \rightarrow \infty$

\begin{tabular}{|l|c|c|c|}
\hline Resolution, time step & $t_{1}$ & $t_{50}$ & $t_{\text {div }}$ \\
\hline Current, $13 \times 16 \times 32 \Delta t=0.01$ & 470.8 & 799.9 & 856.1 \\
\hline Current, $13 \times 16 \times 32 \Delta t=0.001$ & 471.1 & 800.1 & 856.4 \\
\hline Fukagata et. al. [31], $12 \times 16 \times 32 \Delta t=0.0001$ & 2.71 & 34.16 & 167.13 \\
\hline
\end{tabular}

Intereseted readers, can consult [92], where it is explained how and why the Euler equation is bound to diverge for two homogenous directions for all non-trivial initial conditions. 


\section{CHAPTER 5}

\section{DRAG REDUCTION STRATEGIES}

It is far better to foresee even without certainty than not to foresee at all.

Henri Poincare

\subsection{Drag Reduction via Spanwise Oscillations}

Drag reduction due to spanwise oscillations is a well known phenomenon [37, 38, 39, 40, 41, 42, and numerical studies routinely report a drag reduction on the order of $40 \%$. Spanwise oscillations in the case of pipe flow are essentially azimuthal oscillations (about the pipe axis). When the spanwise oscillations are imposed with the frequency $\Omega$ and the amplitude $A$, the azimuthal velocity at the pipe wall satisfies $v_{\text {osc }}(1, \theta, z, t)=A \sin (\Omega t)$. In order to study the effect of the spanwise oscillations using the present approach, we set $v=v_{o s c}-r A \sin (\Omega t)$, so that $v$ still satisfies the homogenous boundary condition at the pipe wall, which is satisfied by the bases employed. With this change of variables (2.5) is transformed into (5.1) and the imposed oscillation acts as a forcing term in the new system;

$$
\begin{aligned}
\partial_{t} \mathbf{u}+(\mathbf{u} \cdot \nabla) \mathbf{u} & =-\nabla p+\frac{1}{\operatorname{Re}_{\mathrm{ref}}} \Delta \mathbf{u}+\mathcal{F} \\
\nabla \cdot \mathbf{u} & =0 \\
\mathbf{u}(1, \theta, z, t) & =0
\end{aligned}
$$


where $\mathcal{F}$ is the forcing due to the oscillation of the pipe wall,

$$
\mathcal{F}=\left(\begin{array}{c}
\left(A \sin (\Omega t) \partial_{\theta} u-2 A \sin (\Omega t)\right) v+r(A \sin (\Omega t))^{2} \\
r \Omega A \cos (\Omega t)+2 u A \sin (\Omega t)+A \sin (\Omega t) \partial_{\theta} v \\
A \sin (\Omega t) \partial_{\theta} w
\end{array}\right)
$$

In this section, the drag reducing effect of spanwise wall oscillations in turbulent regime is investigated. The investigation is carried out in two different settings. One simulation is run at $R e_{\tau}=180$, with the flow driven by a constant pressure gradient. Use of this setting enables the investigation of the effects of wall oscillations on bulk flow. In a separate simulation, the mass flux is kept constant and the driving pressure gradient becomes time dependent. In this setting the Reynolds number is set to $R e=5200$, as in the uncontrolled case. Keeping the mass flux constant enables the observation of changes in wall shear, and the pressure gradient needed to achieve constant flow rate.

\subsubsection{Constant Pressure Gradient Case}

In this case, friction scaling is employed at $R e_{\tau}=180$. The simulation is performed with the same parameters as the fixed wall case, with various values of the oscillation amplitude $A$ and oscillation frequency $\Omega$.

The time evolution of skin friction coefficient scaled by the value for uncontrolled case $(A=0)$ is given in Figure 5.1. In this plot we present the results for a fixed oscillation frequency of $\Omega=6 \pi$ (frequency used in [42]), and vary the oscillation amplitude $A$. The skin friction coefficient, defined by,

$$
c_{f}=\frac{\tau_{w}}{\frac{1}{2} \rho U_{b}^{2}},
$$

decreases from a value of 0.0094 to 0.0061 at $A=20$ in the mean (see Figure 5.1). This amounts to a drag reduction of $35 \%$ consistent with the results previously published [41, 42]. At higher amplitudes, drag reduction seems to level in our computational domain length of 10 radii as reported by [40] where a 20 radii length is used. In Figure 5.2, we present the mean velocity profile for controlled and uncontrolled cases. A summary of our results is presented in Table 5.1. We compute a bulk velocity $U_{b}$ of 14.58 in the case of uncontrolled flow. When spanwise oscillations are imposed on the 


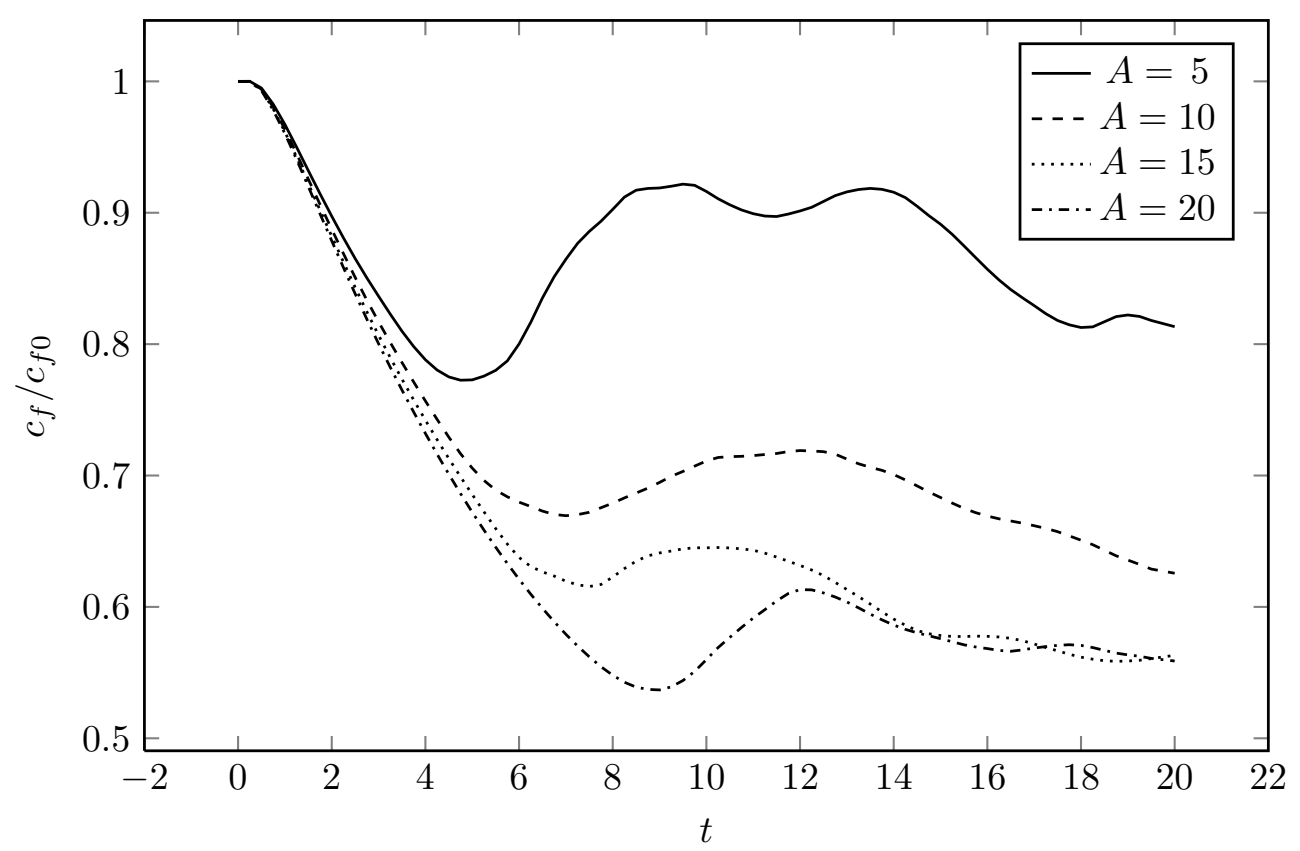

Figure 5.1: Time evolution of skin friction coefficient scaled by uncontrolled $(A=0)$ case. For oscillation amplitudes, $A=2,5,10,15,20$, and frequency $\Omega=6 \pi$

walls, a significant increase in bulk velocity is observed. The average magnitude of the bulk velocity is observed to increase to a value of 18.08, at an oscillation frequency of $6 \pi$, and oscillation amplitude of 20 .

Table 5.1: Comparison of turbulence statistics for controlled and uncontrolled flow, for fixed oscillation frequency $\Omega=6 \pi$

\begin{tabular}{l|cccc} 
& No control & $A=5$ & $A=10$ & $A=20$ \\
\hline$U_{c l} / U_{B}$ & 1.32 & 1.31 & 1.31 & 1.34 \\
$U_{B} / u_{\tau}$ & 14.58 & 15.215 & 16.93 & 18.08 \\
$U_{c l} / u_{\tau}$ & 19.27 & 19.878 & 22.1 & 24.28 \\
$c_{f}$ & 0.00941 & 0.00864 & 0.00698 & 0.00611 \\
$c_{f} / c_{f 0}$ & 1.000 & 0.918 & 0.742 & 0.649 \\
$\operatorname{Re}_{c l}$ & 6864 & 7156 & 7922 & 8722 \\
$\operatorname{Re}_{\operatorname{Re}_{\tau}}$ & 5200 & 5477 & 6094 & 6508 \\
& 180 & 180 & 180 & 180
\end{tabular}




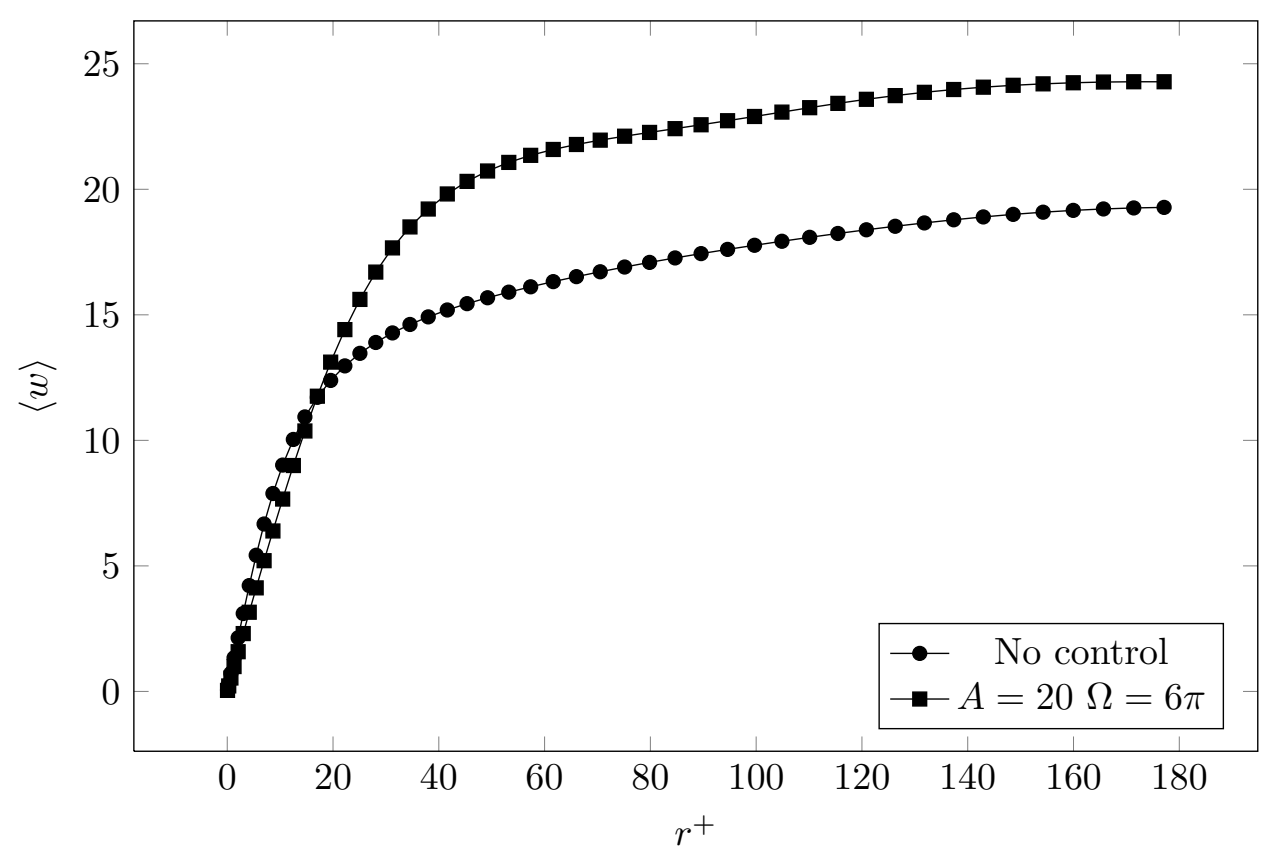

Figure 5.2: Mean streamwise velocity profiles and bulk velocity for controlled $(A \neq 0)$ and uncontrolled $(A=0)$ cases: ' $\square$ ' No control, ' $\triangle$ ' $\mathrm{A}=20, \Omega=6 \pi$.

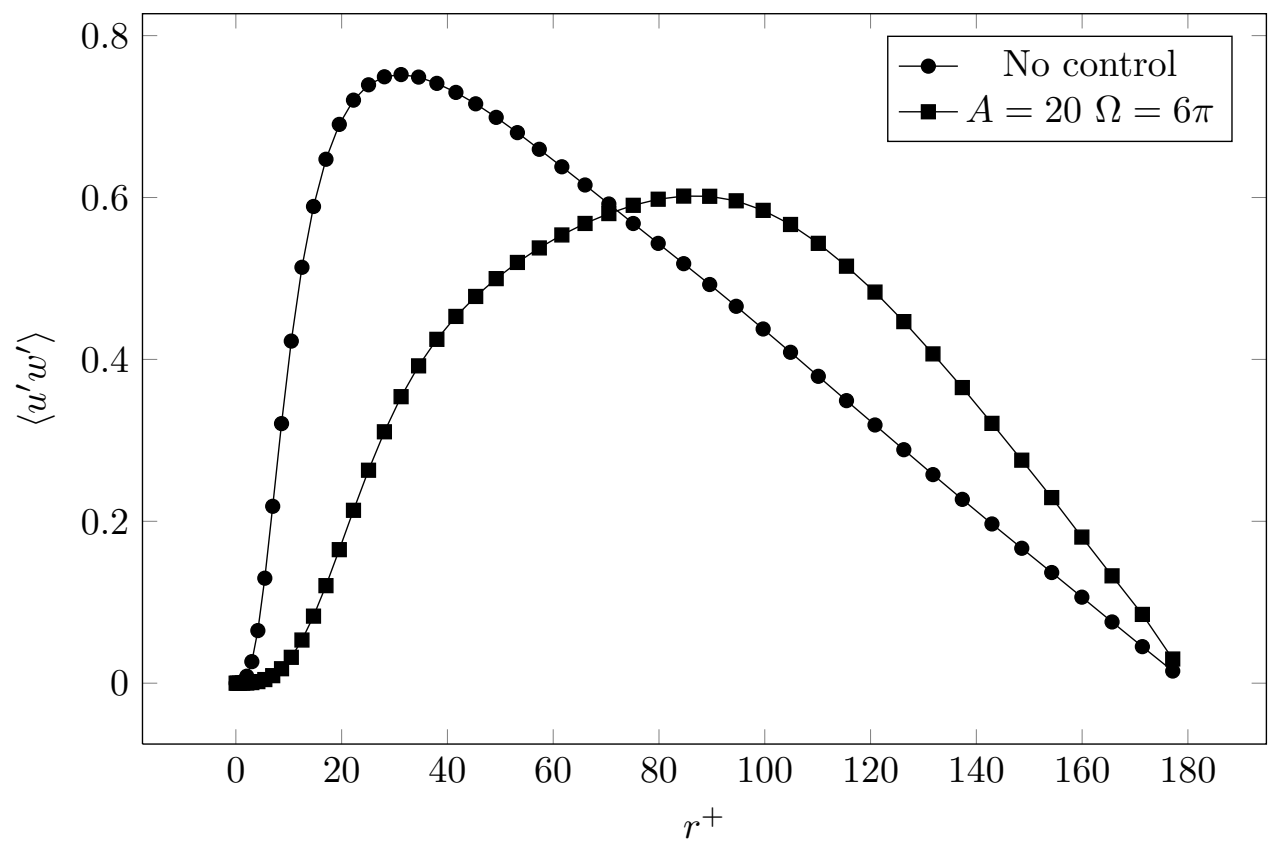

Figure 5.3: Mean Reynolds stress for controlled $(A \neq 0)$ and uncontrolled $(A=0)$ cases: ' $\square$ ' No control, ' $\triangle$ ' $A=20, \Omega=6 \pi$. 


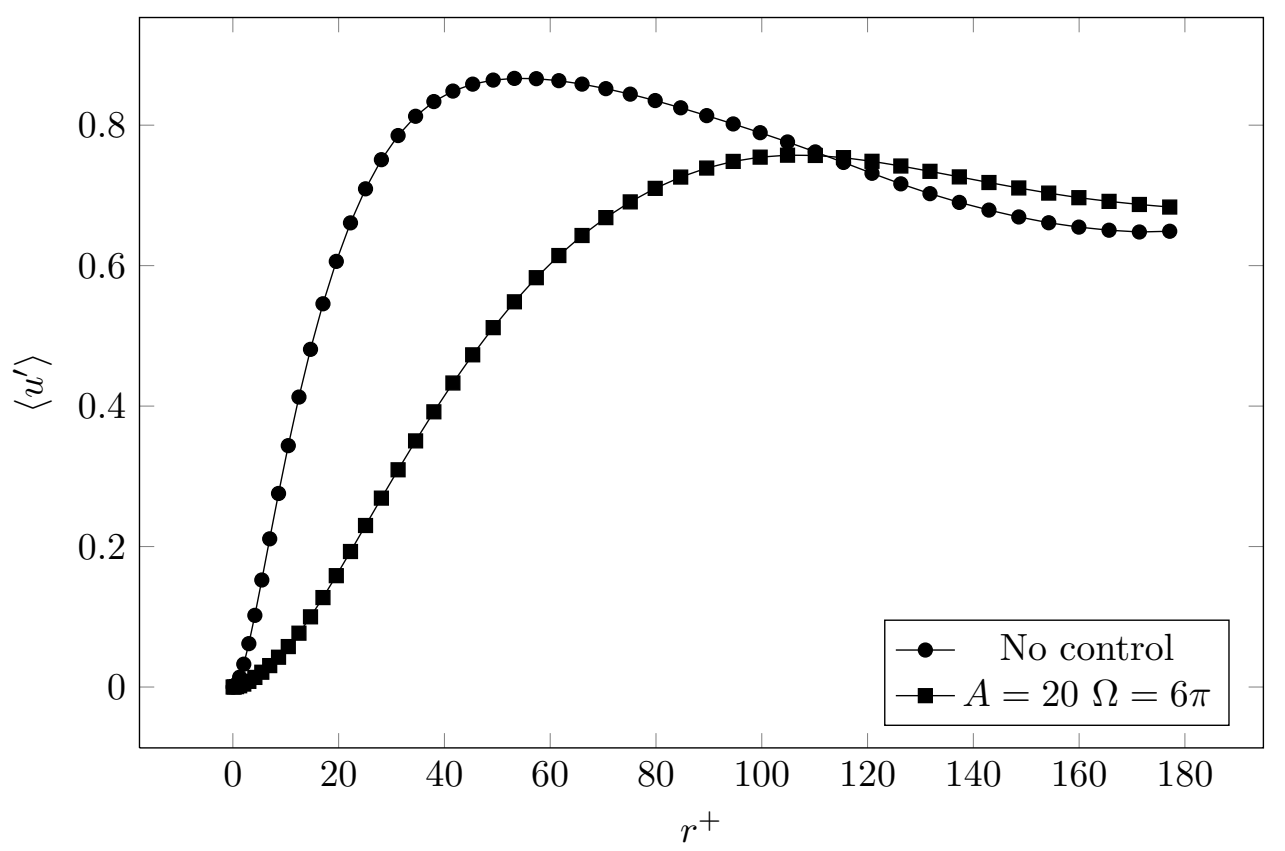

Figure 5.4: Mean $u_{r m s}$ for controlled $(A \neq 0)$ and uncontrolled $(A=0)$ cases: ' $\square$ ' No control, ' $\triangle$ ' $A=20, \Omega=6 \pi$.

Table 5.2: Comparison of turbulence statistics for controlled and uncontrolled flow, for fixed oscillation amplitude $A=10$

\begin{tabular}{l|ccccc} 
& No control & $\Omega=3 \pi$ & $\Omega=4 \pi$ & $\Omega=5 \pi$ & $\Omega=6 \pi$ \\
\hline$U_{c l} / U_{B}$ & 1.32 & 1.33 & 1.32 & 1.33 & 1.31 \\
$U_{B} / u_{\tau}$ & 14.58 & 16.66 & 17.25 & 17.09 & 16.93 \\
$U_{c l} / u_{\tau}$ & 19.27 & 22.08 & 22.75 & 22.68 & 22.10 \\
$c_{f}$ & 0.00941 & 0.00724 & 0.00675 & 0.00685 & 0.00698 \\
$c_{f} / c_{f 0}$ & 1.000 & 0.769 & 0.717 & 0.728 & 0.742 \\
$\operatorname{Re}_{c l}$ & 6864 & 7977 & 8197 & 8168 & 7922 \\
$\operatorname{Re}_{\operatorname{Re}}$ & 5200 & 5998 & 6210 & 6151 & 6094 \\
$\operatorname{Re}_{\tau}$ & 180 & 180 & 180 & 180 & 180
\end{tabular}




\subsubsection{Constant Mass Flux Case}

In addition to the constant pressure gradient case, a simulation in which the mass flux is kept constant, has been performed at $R e=5200$. For this simulation an oscillation amplitude of $A=0.68$ and an oscillation frequency of $\Omega=0.648$ are chosen based on the underlying scaling. These values approximately correspond to $A=20$ and $\Omega=6 \pi$, when scaled as in the uncontrolled constant pressure gradient case. The bulk Reynolds number $R e=5200$, in this case was chosen as it corresponds to earlier simulations using fixed pressure gradient, as well as previous experimental work [30].

To facilitate quantitative comparison with [40], another simulation was performed with $R e=4900$, an oscillation amplitude of $A=0.25$ and an oscillation frequency of $\Omega=0.35$ in bulk units as in 40 .

The results from both simulations are summarized in Table 5.3 in comparison to [40] where an oscillation amplitude of $A \sim 7.1$, and frequency $\Omega \sim 10$ in friction units is used. It is evident that, the pressure gradient required to keep the bulk velocity constant is decreased significantly in the case of wall oscillations. In this case, with relatively high oscillation frequency and amplitude, the order of drag reduction is about \%40. The comparison in Table 5.3 conforms to the behavior of drag reduction which levels as oscillation amplitude and frequency are increased.

Table 5.3: Comparison of turbulence statistics for controlled and uncontrolled flow in the case of constant mass flux. $G_{P}$ denotes the mean pressure gradient required for corresponding laminar flow.

\begin{tabular}{l|cccc} 
& No control & $\begin{array}{c}A=0.68 \\
\Omega=0.648\end{array}$ & $\begin{array}{c}A=0.25 \\
\Omega=0.35\end{array}$ & Quadrio et al. [40] \\
\hline$U_{c l} / U_{B}$ & 1.30 & 1.33 & 1.34 & 1.34 \\
$U_{B} / u_{\tau}$ & 14.30 & 18.31 & 16.37 & 16.60 \\
$U_{c l} / u_{\tau}$ & 18.59 & 24.35 & 21.80 & 22.18 \\
$c_{f}$ & 0.00978 & 0.00597 & 0.0075 & 0.00726 \\
$c_{f} / c_{f 0}$ & 1.000 & 0.610 & 0.746 & 0.736 \\
$G / G_{P}$ & 3.2 & 1.94 & 2.29 & - \\
$G / G_{n c}$ & 1 & 0.604 & 0.722 & - \\
$\operatorname{Re}$ & 6760 & 6916 & 6542 & 6556 \\
$\operatorname{Re}$ & 5200 & 5200 & 4900 & 4900 \\
$\operatorname{Re}$ & 182 & 142 & 149 & 148
\end{tabular}

From Figs. 5.5 and 5.6, it is evident that spanwise wall oscillations do not alter the 


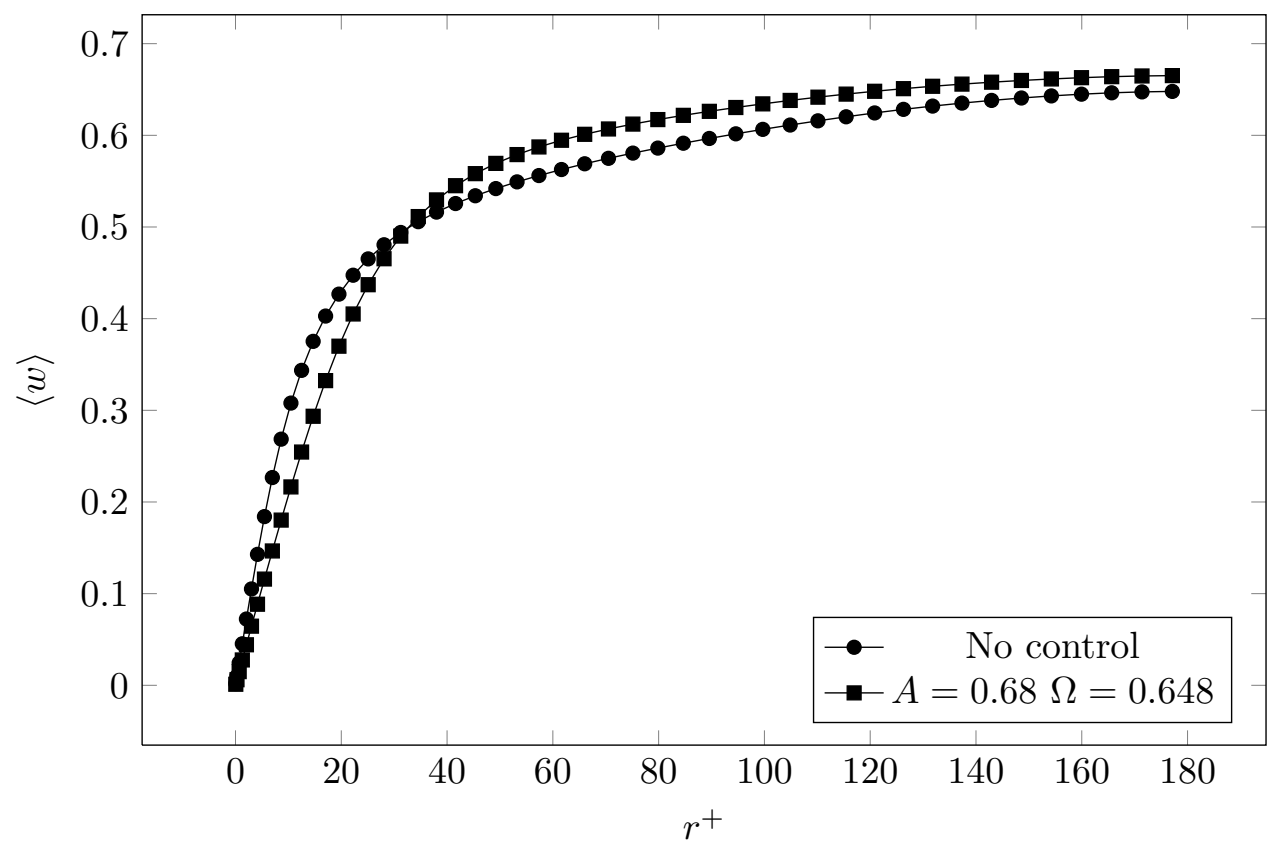

Figure 5.5: Mean streamwise velocity profiles and bulk velocity for controlled $(A \neq 0)$ and uncontrolled $(A=0)$ cases: ' $\square$ ' No control, ' $\triangle$ ' $\mathrm{A}=0.68, \Omega=0.648$.

mean flow as dramatically as in the flow driven by constant pressure gradient. This is to be expected, as in constant pressure case the flowrate is increased significantly in the presence of wall oscillations, in the constant mass flux case however, as the name itself implies, the flowrate is constant. Hence the effect of wall oscillations for the constant mass flux case is the decrease in the magnitude of time dependent forcing $G$, this is depicted in Fig. 5.11. However, as is the case for flow driven by constant pressure gradient, mean Reynolds stress, $\overline{u^{\prime} w^{\prime}}$, and root mean square velocity fluctuations all show both a decrease in peak values, and a centerward shift of the peak location consistent with literature. The Reynolds stress for controlled and uncontrolled flow is given in Fig. 5.7, in this figure, while the peak stress value is decreased from a value of 0.74 to 0.58 as a result of wall oscillations, the peak location is shifted from $r^{+}=33$ to $r^{+}=52$ which is closer to the pipe center. The shifting of peak locations and the accompanying decrease in peak values (except for rms axial velocity, which experiences a slight increase) is plotted in Fig. 5.8 for urms values of radial velocity, Fig. 5.9 for rms values of azimuthal velocity and finally for rms values of axial velocity in Fig. 5.10 , 


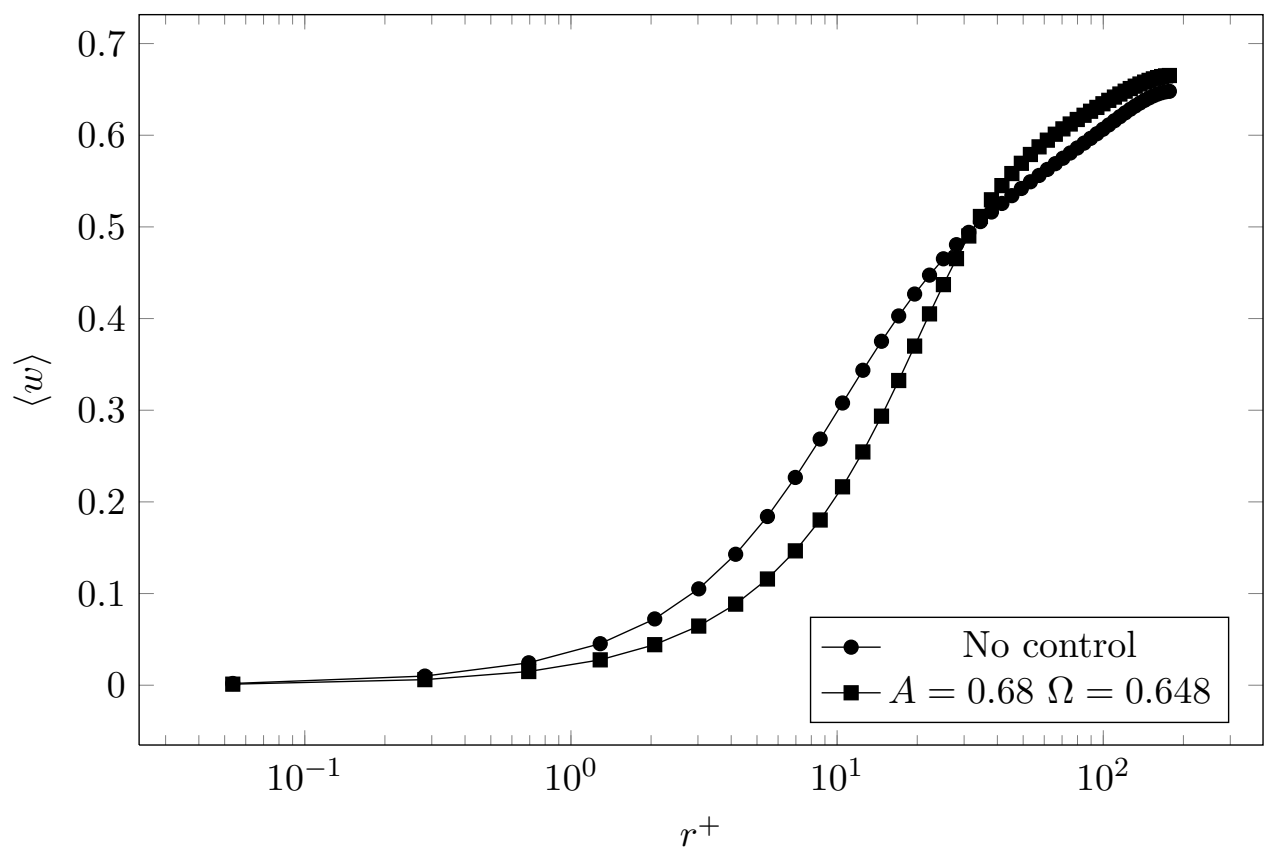

Figure 5.6: Mean streamwise velocity profiles and bulk velocity for controlled $(A \neq 0)$ and uncontrolled $(A=0)$ cases: ' $\square$ ' No control, ' $\triangle$ ' $\mathrm{A}=0.68, \Omega=0.648$.

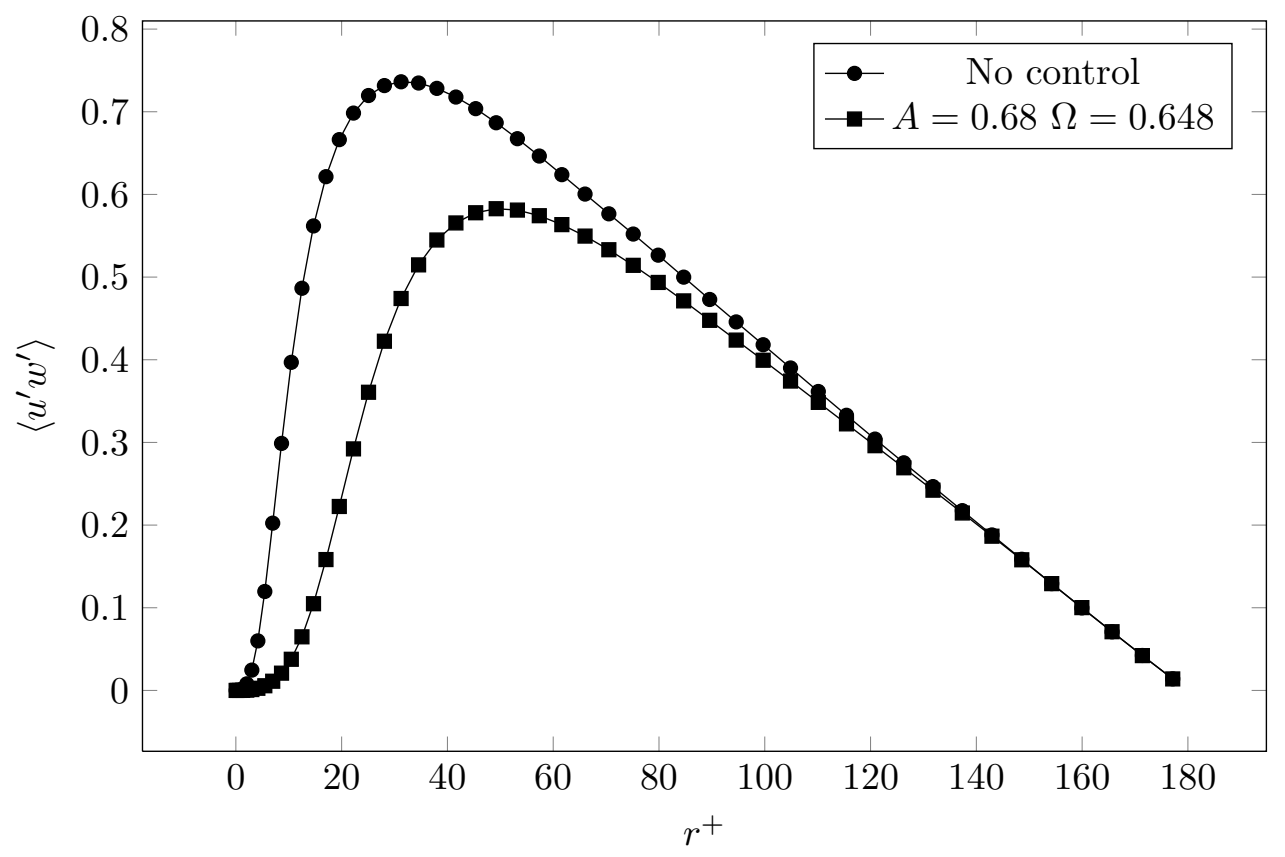

Figure 5.7: Mean Reynolds stress for controlled $(A \neq 0)$ and uncontrolled $(A=0)$ cases: ' $\square$ ' No control, ' $\triangle$ ' $A=0.68, \Omega=0.648$. 


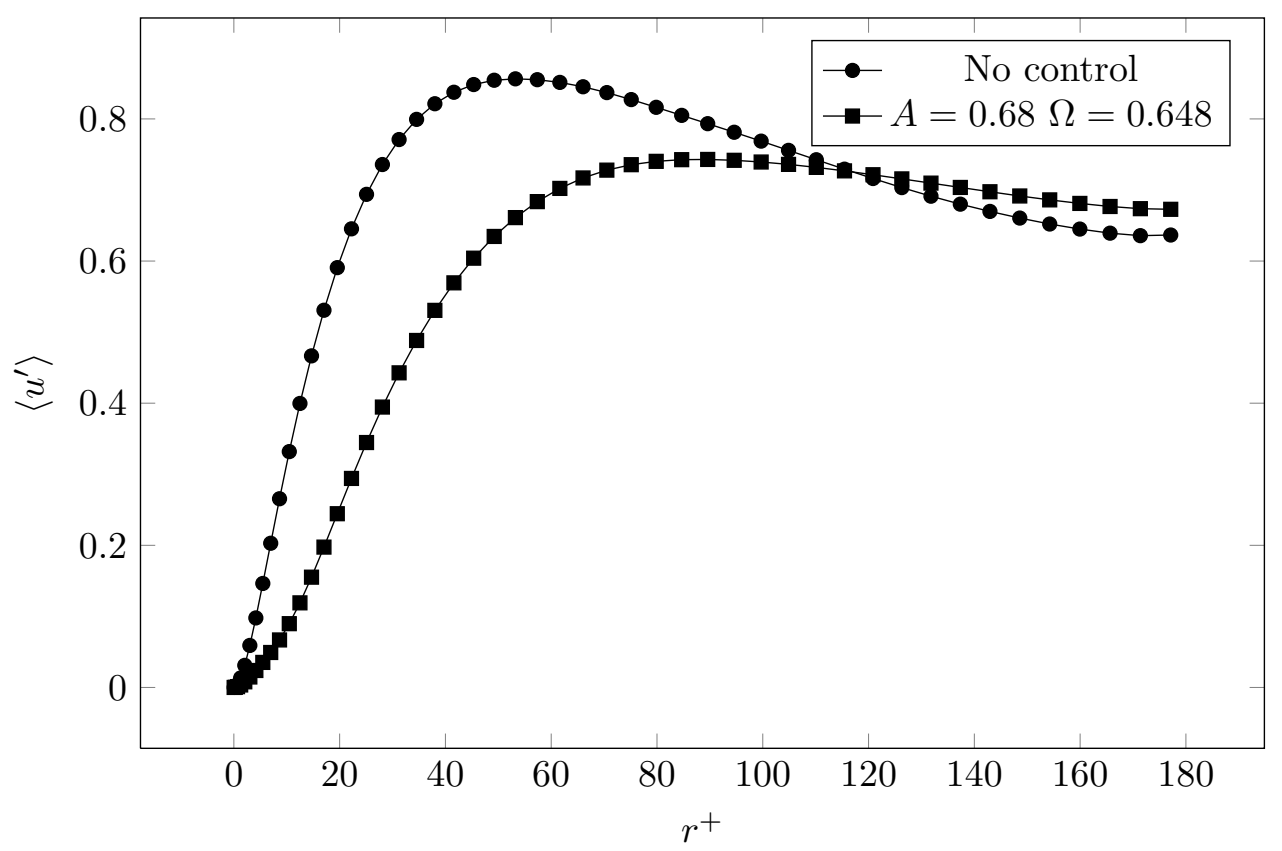

Figure 5.8: rms u for controlled $(A \neq 0)$ and uncontrolled $(A=0)$ cases: ' $\square$ ' No control, ' $\triangle^{\prime} \mathrm{A}=0.68, \Omega=0.648$.

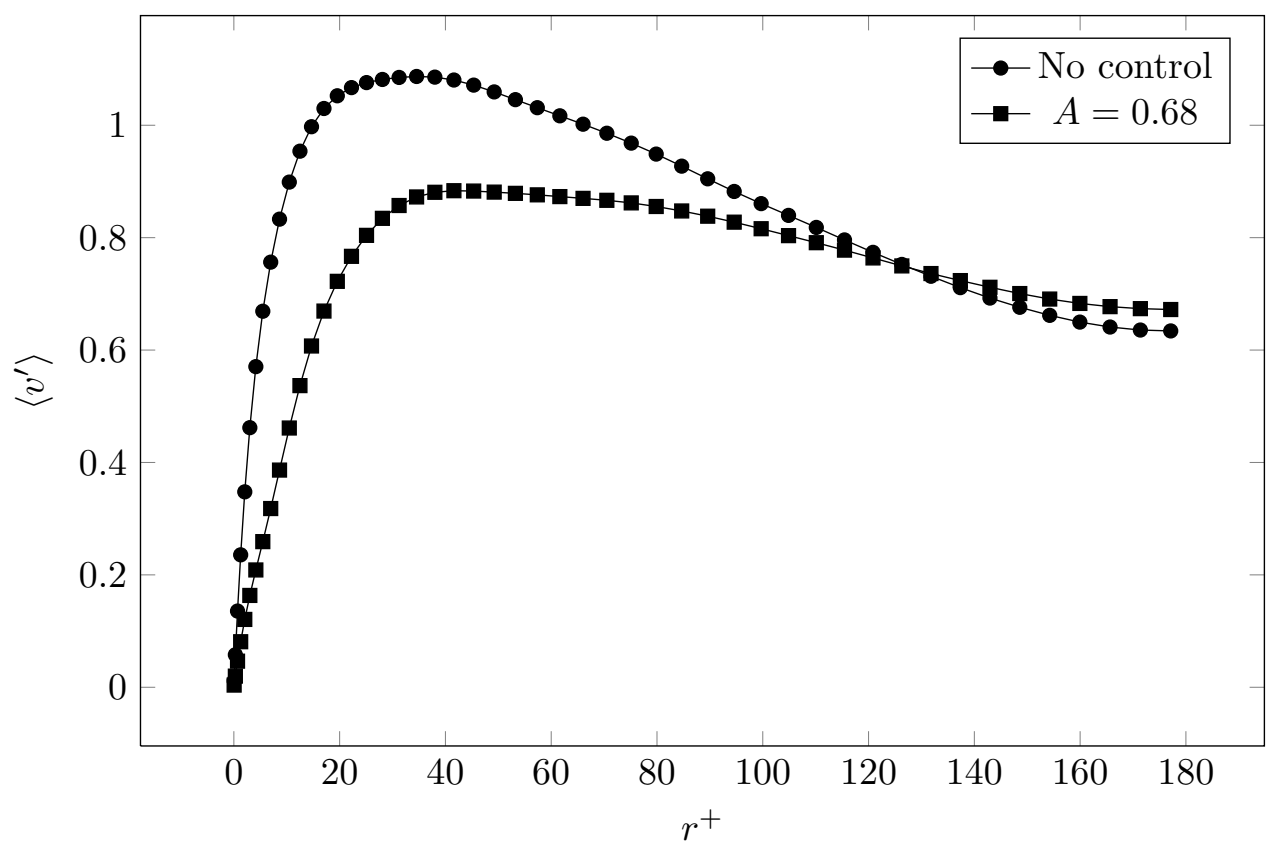

Figure 5.9: $\mathrm{rms} \mathrm{v}$ for controlled $(A \neq 0)$ and uncontrolled $(A=0)$ cases: ' $\square$ ' No control, ' $\triangle$ ' $\mathrm{A}=0.68, \Omega=0.648$. 


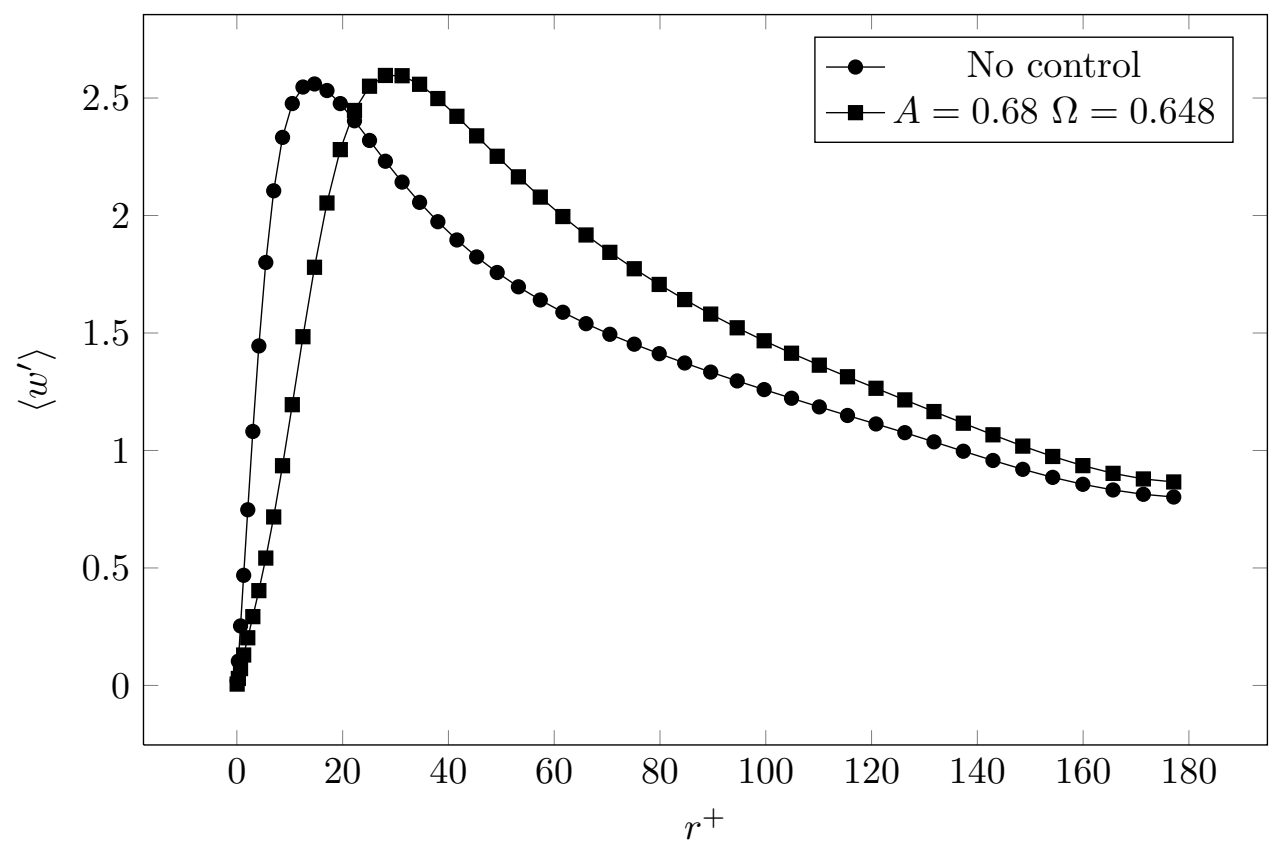

Figure 5.10: rms w for controlled $(A \neq 0)$ and uncontrolled $(A=0)$ cases: ' $\square$ ' No control, ' $\triangle$ ' $\mathrm{A}=0.68, \Omega=0.648$.

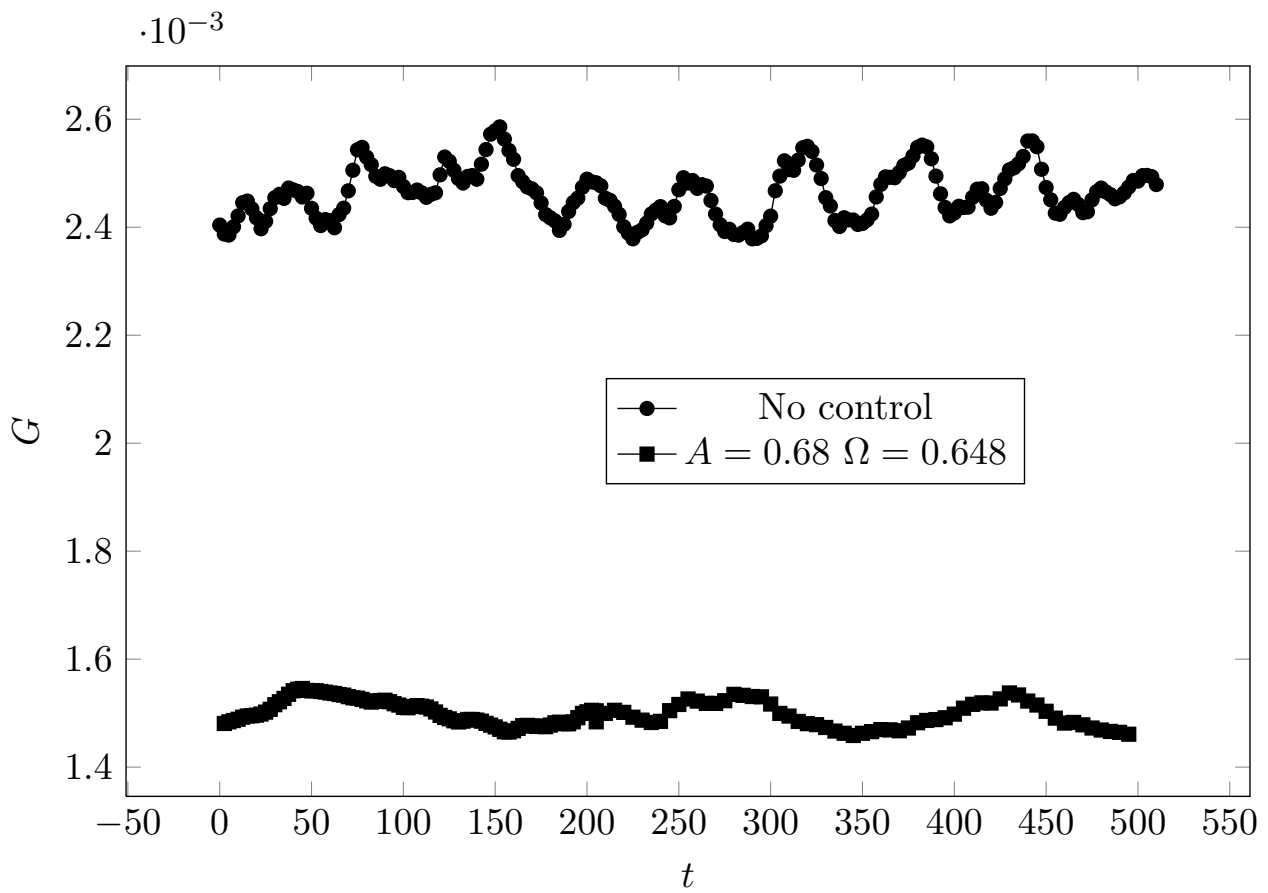

Figure 5.11: Pressure gradient $\mathrm{g}$ for controlled $(A \neq 0)$ and uncontrolled $(A=0)$ cases: ' $\square$ ' No control, ' $\triangle$ ' $A=0.68, \Omega=0.648$. 


\subsection{Phase Randomization}

The main idea behind drag reduction via phase randomization is that, a statistically significant drag reduction can be obtained via distrupting the wave-like structures in the flow. This impedes the energy transfer between the wave like structures and the rolls present in the flow field [44. To distrupt these structures, a periodic phase randomization is applied to some of the wave modes. This approach was first undertaken for the case of channel flow by Sirovich and Handler [44] in a numerical manner and later backed by an experimental study [45]. Both the mentioned works report a drag reduction of upto around $50 \%$, depending on the mode selection and the distruption frequency. It was also shown that randomizing the phases of high wave number modes or rolls results in a drag increase.

Conceptually phase randomization is very simple, considering the expansion for velocity $(3.6)$.

$$
\mathbf{u}(r, \theta, z, t)=\sum_{l=-Q}^{Q} \sum_{n=-N}^{N} \sum_{m=0}^{M} a_{l n m}^{(1)}(t) \Phi_{l n m}^{(1)}(r, \theta, z)+a_{l n m}^{(2)}(t) \Phi_{l n m}^{(2)}(r, \theta, z)
$$

where $a_{l n m}$ are the complex time dependent expansion coefficients. At given intervals a random shift of $\phi_{n m}$ is introduced, such that,

$$
a_{l n m} \rightarrow e^{i \phi_{n m}} a_{l n m}
$$

The operation in (5.3) can also be seen as a velocity dependent forcing (5.4), obviously this operation preserves continuity, furthermore it does not change the flow energy. Thus phase randomization does no work on the flow [44].

$$
\partial_{t} \mathbf{u}+(\mathbf{u} \cdot \nabla) \mathbf{u}=-\nabla p+\frac{1}{\operatorname{Re}_{\mathrm{ref}}} \Delta \mathbf{u}+\sum_{T} F_{T}(\mathbf{u}) \delta(t-T) .
$$

To reiterate, the application of phase randomization process on a given flowfield does not alter the mean flow statistics, such as the average velocity and vorticity profile. The only visible effect is the rotation or translation of vortices with respect to each other. 
Typical large eddy turnover time for the flow can be estimated as $2 r / u^{\prime}$, where $u^{\prime}$ is a typical rms velocity fluctuation level [44]. For the uncontrolled flow the peak streamwise fluctuation is calculated to be on the order of 2.5 in our case. As the friction Reynolds number was calculated as 175 for this case, the large eddy turnover time is calculated as $T_{e}^{+}=140$. Our simulations were run for 50 large eddy turnovers (corresponding to roughly 40 friction units or 1120 bulk time units) before phase randomization was turned on, following [44]

The random phase shifts are applied to the low band of modes such that,

$$
1<l<8 \quad|n|<5
$$

this corresponds, roughly, to

$$
\sqrt{k_{l}^{2}+k_{n}^{2}} \leq k_{\max } / 6
$$

as in [44], where $\mathrm{k}$ denotes the wave number and $k_{\max }=75 \times 2 \pi / L_{z}, 75$ being the number of positive Fourier modes selected along the axial direction in this study. The application of phase randomization does not effect the flow energy and to continue integrating in time two techniques are possible. One method is to perform the phase randomization and continue time integration using a self starting scheme (such as Runge-Kutta), the other is to apply the phase randomization precedure to all the time levels stored for the IMEX type scheme detailed in Sec. 3.3. Application of the same phase randomization to all the stored time levels results effectively in modification of Eq. (3.9), as

$$
\begin{aligned}
e^{i \phi_{n m}}(11 \mathbf{A}-6 \Delta t \mathbf{B}) a^{(k+1)}= & e^{i \phi_{n m}} \mathbf{A}\left(18 a^{(k)}-9 a^{(k-1)}+2 a^{(k-2)}\right) \\
& -e^{i \phi_{n m}} \Delta t\left(18 b^{(k)}-18 b^{(k-1)}+6 b^{(k-2)}\right),
\end{aligned}
$$

hence the validity of the time solver is not violated. The tests reveal the two mentioned methods yield the same turbulent statistics, and for performance reasons the latter approach (application of the same phase randomization to all the stored time levels) has been selected for performance reasons. 


\subsubsection{Constant Pressure Gradient Case}

The effects of phase randomization were investigated at friction Reynolds number $R e_{\tau}=180$ with a constant pressure gradient applied along the pipe axis. The findings are summarized in Table 5.4. Also presented is a brief comparison of turbulence statistics in comparison to uncontrolled case and to the oscillatory wall case 5.5 . The main difference between wall oscillations and phase randomization is the fact that, with the mode selection given in Sec. 5.2, the transition from re-laminarization to drag-reduced turbulent flow is quite abrupt. It is worthwhile to point out that, even though the drag reduction values for phase randomization appear lower than those obtained by spanwise wall oscillations, the maximum net power saved amounts to about $8 \%$ with this method, which is comparable to the values obtained via phase randomization.

Table 5.4: Effect of phase randomization for constant axial pressure gradient

\begin{tabular}{|l|l|c|}
\hline Time step & $\Delta_{\text {phase }} t^{+}$ & Percent Drag reduction \\
\hline$N_{s}=500 \Delta t$ & 22.48 & Re-laminarization \\
\hline$N_{s}=600 \Delta t$ & 26.98 & Re-laminarization \\
\hline$N_{s}=616 \Delta t$ & 27.7 & 8 \\
\hline$N_{s}=875 \Delta t$ & 32.32 & 4 \\
\hline
\end{tabular}

Table 5.5: Comparison of turbulence statistics for controlled and uncontrolled flow, for fixed oscillation frequency $\Omega=6 \pi$ and phase randomization

\begin{tabular}{l|ccccc} 
& No control & $A=5$ & $A=10$ & $A=20$ & $\begin{array}{c}\text { Phase randomization } \\
T^{+}=27.7\end{array}$ \\
\hline$U_{c l} / U_{B}$ & 1.32 & 1.31 & 1.31 & 1.34 & 1.30 \\
$U_{B} / u_{\tau}$ & 14.58 & 15.215 & 16.93 & 18.08 & 15.23 \\
$U_{c l} / u_{\tau}$ & 19.27 & 19.878 & 22.1 & 24.28 & 19.79 \\
$c_{f}$ & 0.00941 & 0.00864 & 0.00698 & 0.00611 & 0.0086224 \\
$c_{f} / c_{f 0}$ & 1.000 & 0.918 & 0.742 & 0.649 & 0.916 \\
$\operatorname{Re}_{c l}$ & 6864 & 7156 & 7922 & 8722 & 7124 \\
$\operatorname{Re}$ & 5200 & 5477 & 6094 & 6508 & 5483 \\
$\operatorname{Re}_{\tau}$ & 180 & 180 & 180 & 180 & 180
\end{tabular}




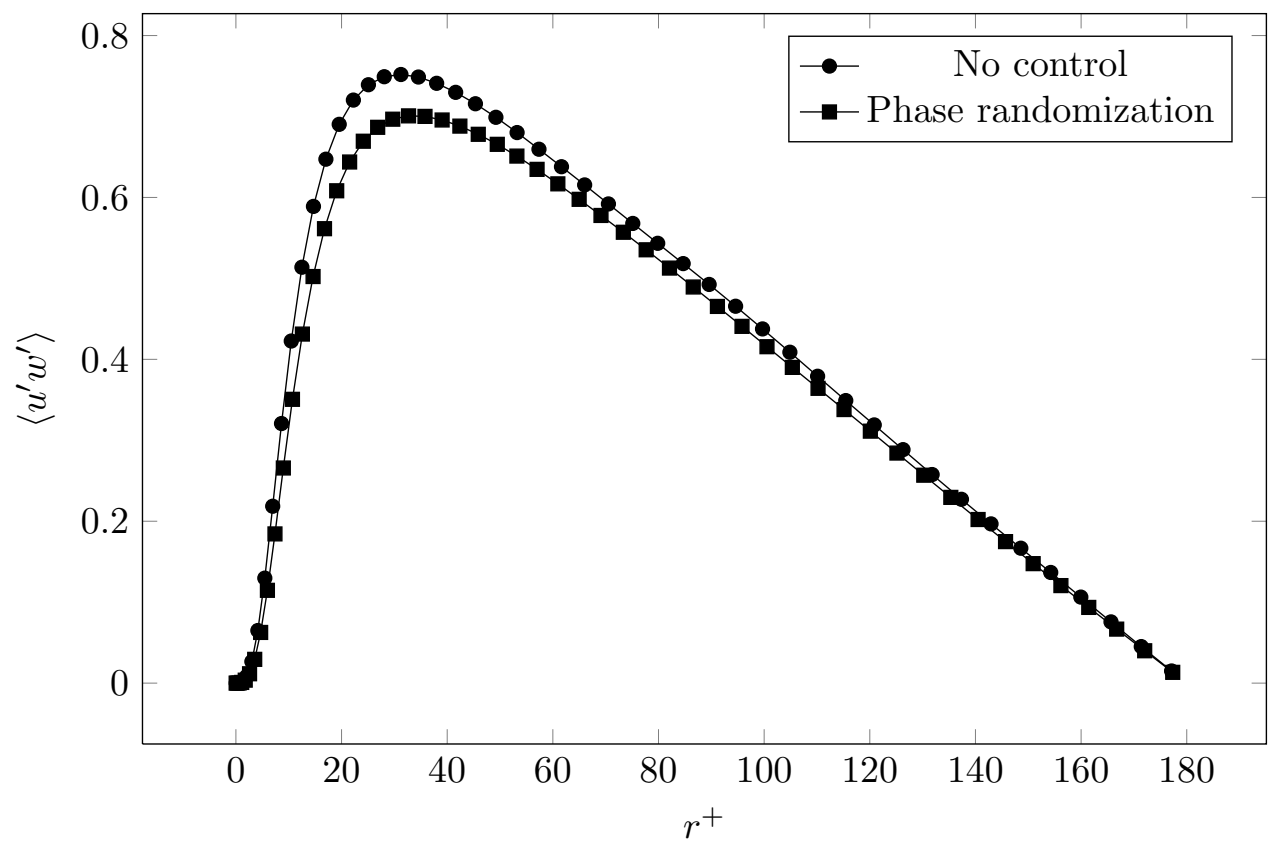

Figure 5.12: Mean Reynolds stress for controlled and uncontrolled cases: ' $\square$ ' No control, ' $\triangle$ ' Phase randomization, constant pressure gradient.

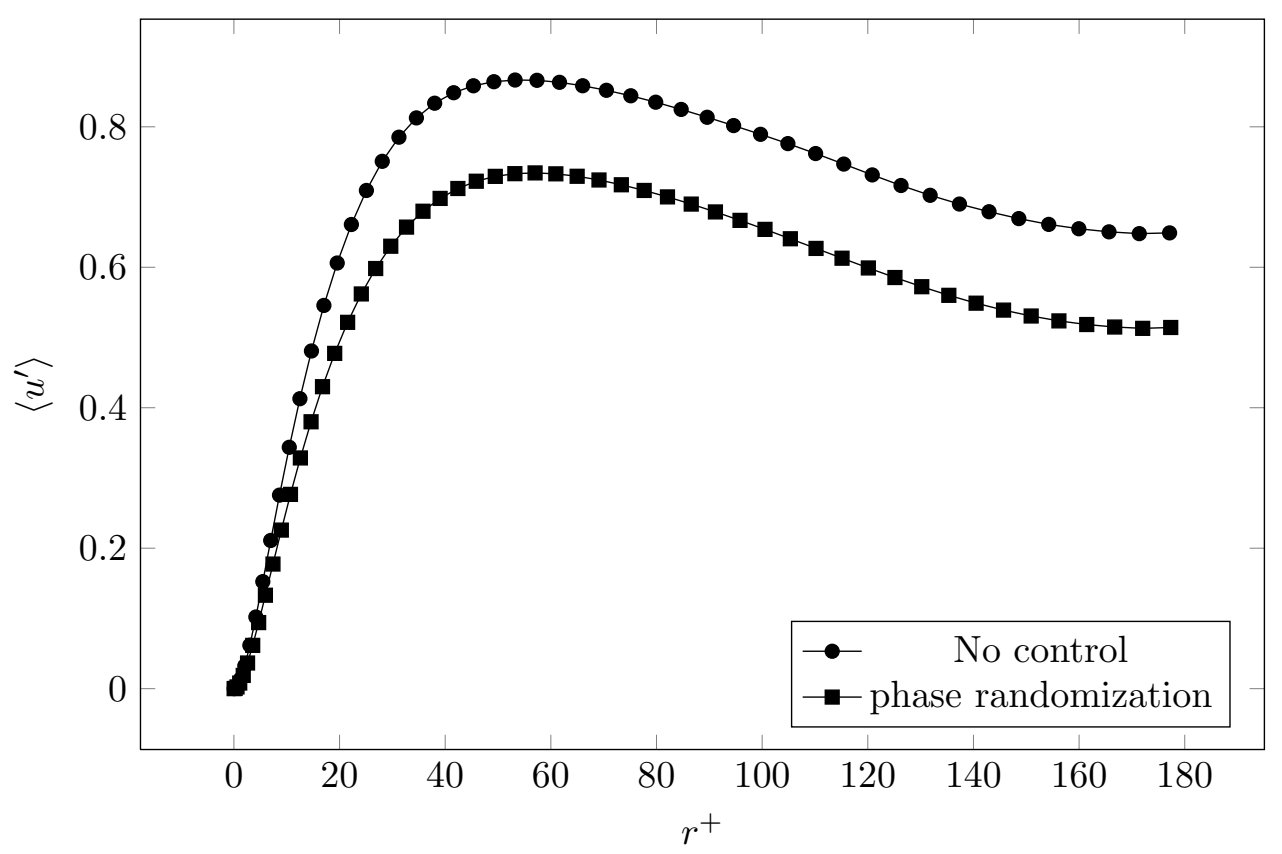

Figure 5.13: Mean urms for controlled $(A \neq 0)$ and uncontrolled $(A=0)$ cases: ' $\square$ ' No control, ' $\triangle$ ' phase randomization. 


\subsubsection{Constant Mass Flux Case}

For the constant mass flux case, a bulk Reynolds number of 4900 was chosen, to facilitate comparison with the oscillatory wall case. The findings are summarized in Table 5.6. In comparison to constant pressure driven flow in Sec. 5.2.1, with mass flux fixed, the transition between re-laminarization and drag reduced turbulent flow is less abrupt, however the maximum achievable drag reduction is on the order of $20 \%$, which is lower than the maximum levels attainable with spanwise wall oscillations. One major point in comparing the two drag redcution strategies however is the fact that, phase randomization is a passive mechanism and in real life applications the drag reduction reported here will likely be the net power saved however in the case of wall oscillations, which constitute active flow control, this is not the case.

Table 5.6: Effects of phase randomization, constant mass flux

\begin{tabular}{|l|l|c|}
\hline Step & Time wall units & Percent Drag reduction \\
\hline$N_{s}=500 \Delta t$ & 13.23 & Re-laminarization \\
\hline$N_{s}=850 \Delta t$ & 18.682 & Re-laminarization \\
\hline$N_{s}=875 \Delta t$ & 19.85 & 20 \\
\hline$N_{s}=1000 \Delta t$ & 23.158 & 7 \\
\hline$N_{s}=1250 \Delta t$ & 26.46 & 4 \\
\hline
\end{tabular}

Table 5.7: Turbulence statistics for controlled and uncontrolled flow in the case of constant mass flux. $G_{P}$ denotes the mean pressure gradient required for corresponding laminar flow.

\begin{tabular}{l|ccc} 
& No control & $\begin{array}{c}A=0.25 \\
\Omega=0.35\end{array}$ & Phase randomization $T^{+}=19.85$ \\
& & 1.34 & \\
\hline$U_{c l} / U_{B}$ & 1.31 & 16.37 & 1.32 \\
$U_{B} / u_{\tau}$ & 14.08 & 21.80 & 20.86 \\
$U_{c l} / u_{\tau}$ & 18.45 & 0.0075 & 0.008012 \\
$c_{f}$ & 0.01008 & 0.795 \\
$c_{f} / c_{f 0}$ & 1.000 & 0.746 & 2.54 \\
$G / G_{P}$ & 3.17 & 2.29 & 0.78 \\
$G / G_{n c}$ & 1 & 0.722 & 6468 \\
$\operatorname{Re}_{c l}$ & 6419 & 6542 & 4900 \\
$\operatorname{Re}_{\operatorname{Re}}$ & 4900 & 4900 & 155
\end{tabular}




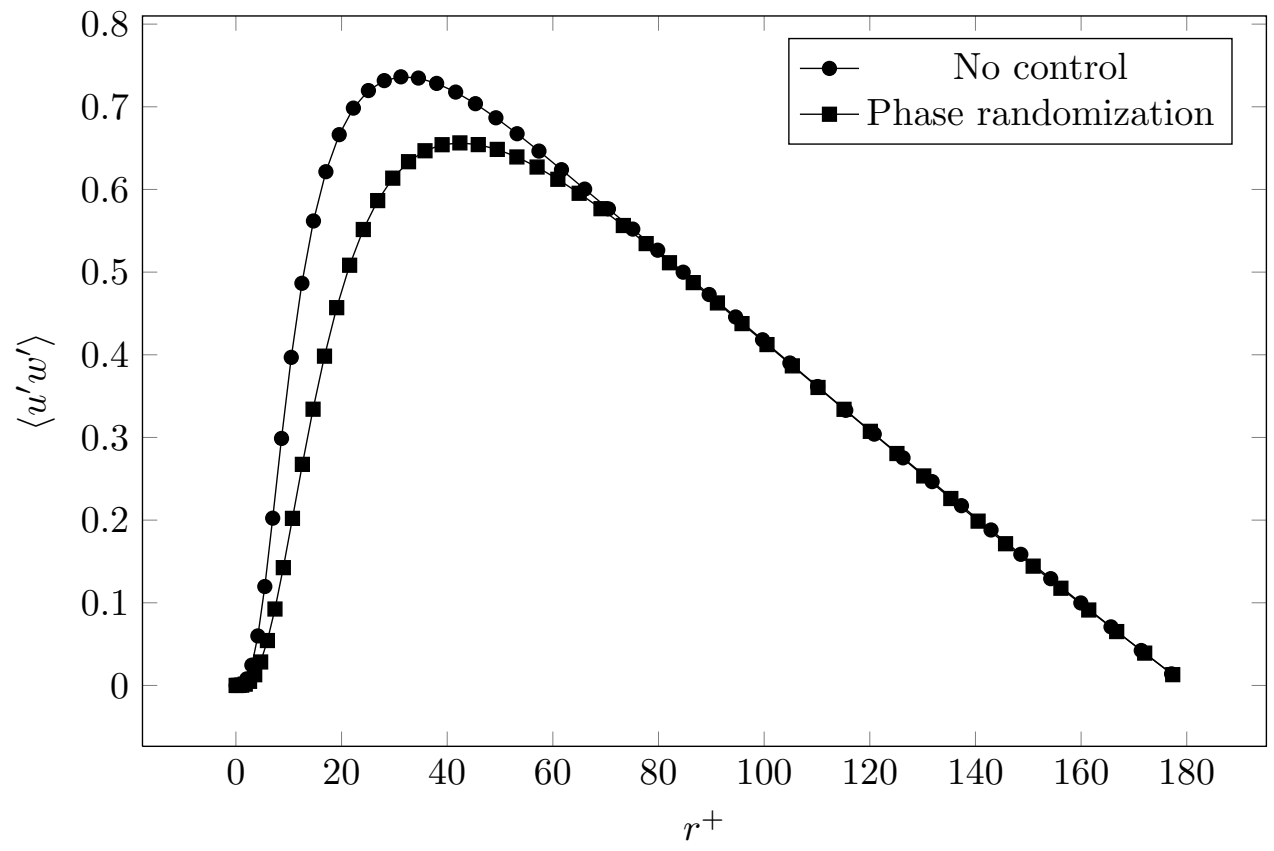

Figure 5.14: Mean Reynolds stress for controlled $(A \neq 0)$ and uncontrolled $(A=0)$ cases: ' $\square$ ' No control, ' $\triangle$ ', phase randomization, constant mass flux.

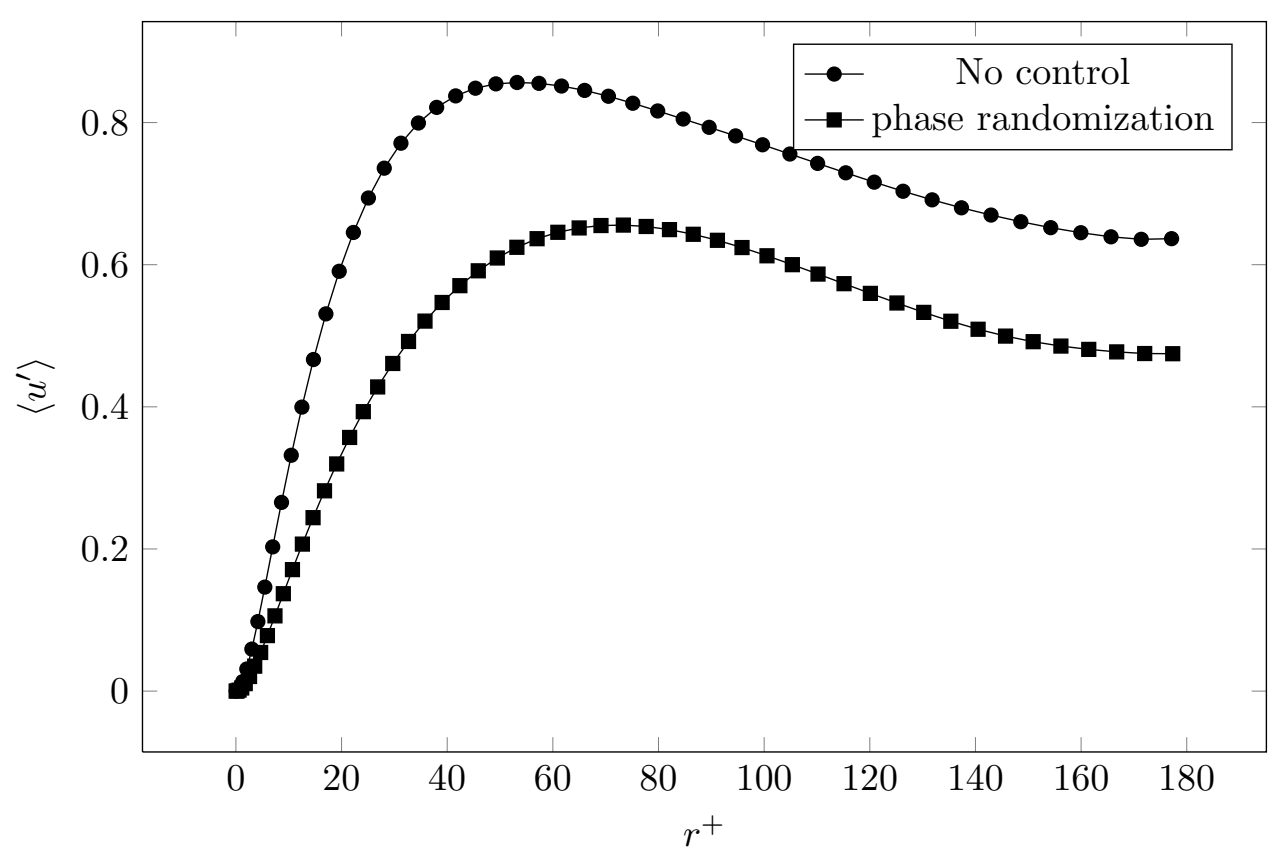

Figure 5.15: rms u for controlled and uncontrolled cases: ' $\square$ ' No control, ' $\triangle$ ' phase randomization, constant mass flux. 


\subsubsection{Drag Reduction Summary}

In this section, common features appearing in the two drag reduction methodologies tested are presented. For the sake of brevity only the results from constant mass flux driven flow are presented in the section, as qualitatively there appears to be negligible difference between these two forcing mechanisms.

As this section presents vorticity plots from the simulations for the first time, it is worthwhile to remind the reader, that vorticity in the cylindrical coordinates is given in $(5.7)$

$$
\vec{\omega}=\left(\frac{1}{r} \frac{\partial w}{\partial \theta}-\frac{\partial v}{\partial z}\right) \hat{r}+\left(\frac{\partial u}{\partial z}-\frac{\partial w}{\partial r}\right) \hat{\theta}+\left(\frac{1}{r} \frac{\partial}{\partial r}(r v)-\frac{1}{r} \frac{\partial u}{\partial \theta}\right)
$$

As a means of underlying the effect of drag reduction techniques applied, we present the instantaneous steam traces emanating from a line perpendicular to the pipe axis at the pipe entrance. Fig. 5.16(a) presents the streamlines for the uncontrolled flow at $t=800$ in bulk units, while Fig. 5.16(b) and Fig. 5.16(c) depicts the streamlines for oscillatory control and phase randomization respectively. It is immediately evident from Fig. 5.16(a) and Fig. 5.16(c) that, the phase randomization procedure does not seem to affect the velocity field, as the flow rate is fixed, however in the case of wall oscillations, it is readily observable from Fig. $5.16(\mathrm{~b})$ that the underlying streamline are effected considerably. It might be stated that spanwise wall oscillations, naturally, enhance mixing while concurrently decrease drag, even though these two phenomenon are generally reported not to occur simultaneously [45. This property might be the deciding factor in choosing a drag reduction mechanism in real time applications.

The effects of both drag reduction mechanisms investigated were found to be qualitatively similar in transferring turbulent activity away from the wall, which was first observed for wall oscillations in [38, 93]. Both mechanisms presented, showed a tendency to suppress root mean square velocity components in azimuthal and radial directions, as well as shift the positions of the corresponding maxima away from the wall (Figs 5.17(a) -5.18(c) . For the radial component of average root mean square velocity (Figs $5.17(\mathrm{a})$ - $5.17(\mathrm{c})$, it was observed that phase randomization was 


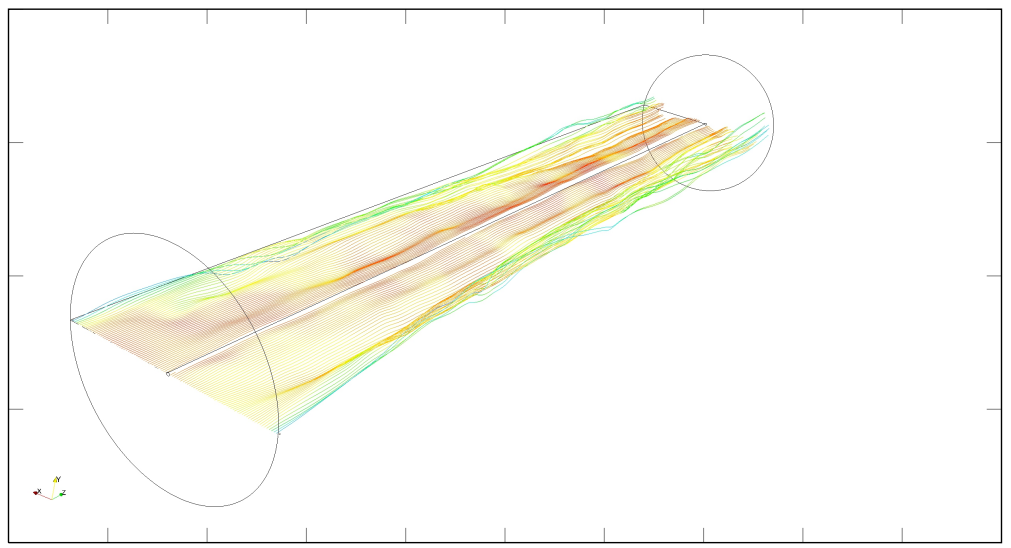

(a) Stream lines at $t=800$ constant mass flux, no control

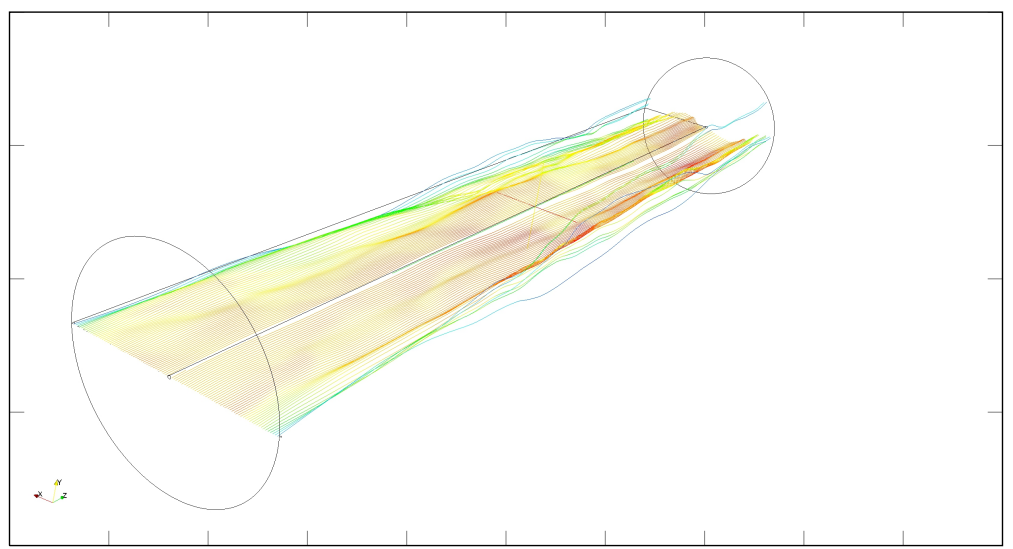

(b) Stream lines at $\mathrm{t}=800$, constant mass flux, phase randomization with $T^{+}=875$

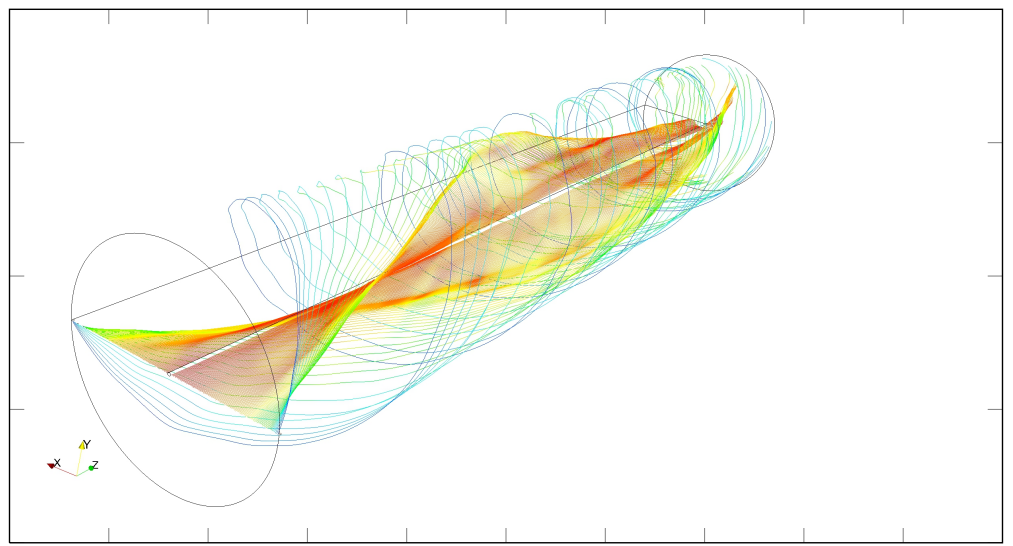

(c) Stream lines at $\mathrm{t}=800$, constant mass flux, oscillatory wall, $\mathrm{A}=0.5, \Omega=$ 0.35

Figure 5.16: Stream lines at $\mathrm{t}=800$ constant mass flux, controlled and uncontrolled cases 
more effective in comparison with spanwise wall oscillations. For the average root mean square velocity in the azimuthal direction (Figs. 5.18(a)-5.18(c) however spanwise wall oscillations perform better in suppressing azimuthal velocity when compared to phase randomization. The stronger suppression of azimuthal velocities in the case of wall oscillations is attributable to the azimuthal forcing nature of this drag reduction technique [38, 93. The region in which the drag reduction methods were effective was found to be the wall region as anticipated.

With regards to vortical activity, changes similar to those found in radial and azimuthal root mean square velocity components was observed (Figs. 5.19(a)-5.21(c)]. For the vorticity components, both a drop in magnitude and a centerward shift of maximum locations resulted from the use drag reduction mechanisms. Among the differences, wall oscillations were found to be better in suppressing radial vortex activity near the wall, and in pushing the activity center away from the pipe wall (Figs. $5.19(\mathrm{a})-5.19(\mathrm{c})$. However, even though phase randomization was a comparatively worse performer in these regards, application of phase randomization results in an almost uninterrupted prominent low radial vorticity region in the center. Looking at the components of radial vorticity (5.7), the more aggressive nature of wall oscillations against radial vorticity components is readily explicable by its azimuthal forcing nature.

The near wall prominence of the drag reduction techniques is most evident in the case of azimuthal vorticity components (Figs. 5.20(a) -5.20(c) $)$. In this case, the thin near wall region of high azimuthal vorticity is considerably weakened by the application of both wall oscillations and phase randomization, phase randomization being to more effective, this is most probably related to its effectiveness against radial component of average root mean square velocity.

The axial vortices, or the axial vorticity components respond differently to spanwise oscillations in comparison to other two vorticity components (Figs 5.21(a), 5.21(b)) The r-dependent azimuthal velocity induced by wall oscillations is shown to result in a high vorticity near wall layer (5.7). This however is less relevant in terms of drag reduction, as the suppression of cross components of vorticity (i.e. radial and azimuthal) have been cited as the principal cause of drag reduction for most drag 
reduction techniques [38, 93]. In contrast to wall oscillations, phase randomization results in a significant drop in overall axial vorticity in the flow field, this however, as mentioned before is not as relevant for drag reduction as the effects previously mentioned.

As a final visualization of the effects of the control methods introduce, the centerward shift in vortices in the presence of drag control is visualized in Fig. 5.22(a), Fig. 5.22(b) and Fig. 5.22(c), In these graphs radial vorticity in an outer cylindrical portion of the pipe is depicted. The inner radius of this outer shell is $r=0.95$ when scaled with the pipe radius, this corresponds to $r^{+} \sim 9$, in wall units, and represents the so-called wall region [30]. The removal of radial vortical activity from the wall region is especially dramatic in the case of wall oscillations (Fig. 5.22(b) , as earlier cross-sectional plots suggest (Fig. 5.19(b)) . Application of phase randomization shows elimination of radial vortical activity, albeit at a lower rate (Fig. 5.19(c)]

Overall, drag-reducing effect of phase randomization in pipe flow was found to be similar to that of wall-oscillations, namely the suppression of cross-flow and crossvorticity components. Differences exist however, as phase randomization is more proficient in suppressing radial cross-flow component and azimuthal vorticity component than spanwise wall oscillations. 


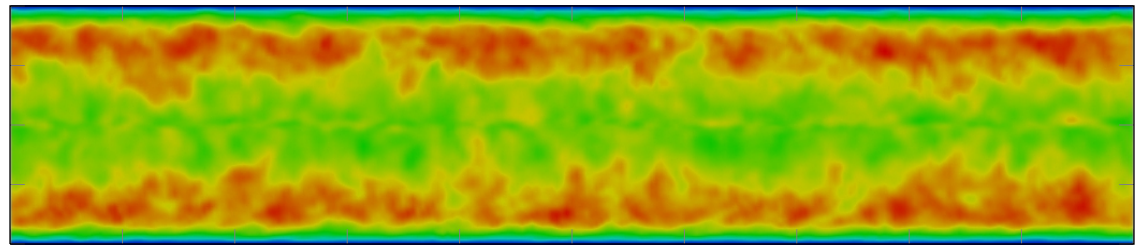

(a) RMS u velocity, time average, constant mass flux, no control

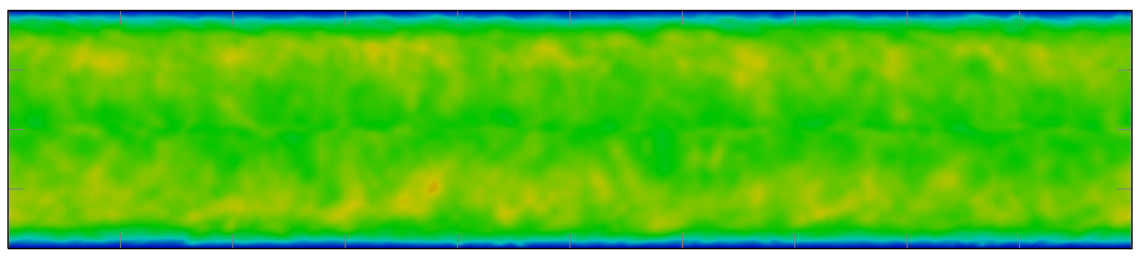

(b) RMS u velocity, time average, constant mass flux, oscillatory wall, $\mathrm{A}=0.5, \Omega=$ 0.35

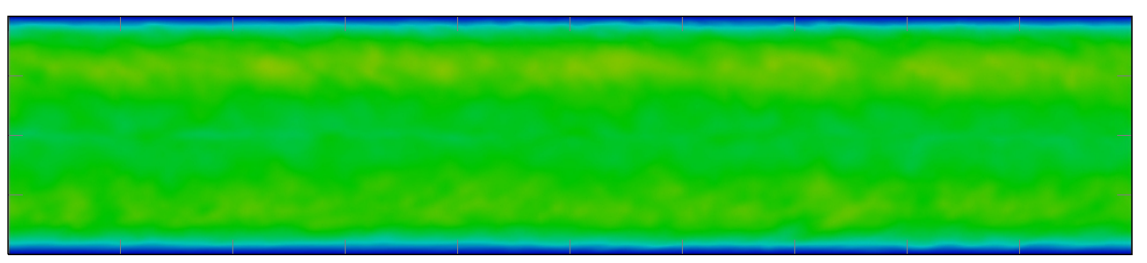

(c) RMS u velocity, time average, constant mass flux, phase randomization with $T^{+}=$ 875

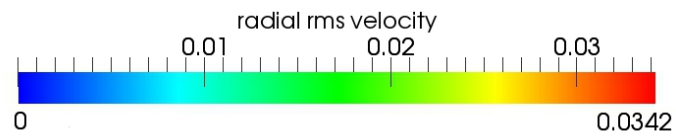

Figure 5.17: RMS radial velocity, time average, constant mass flux, controlled and uncontrolled cases. 


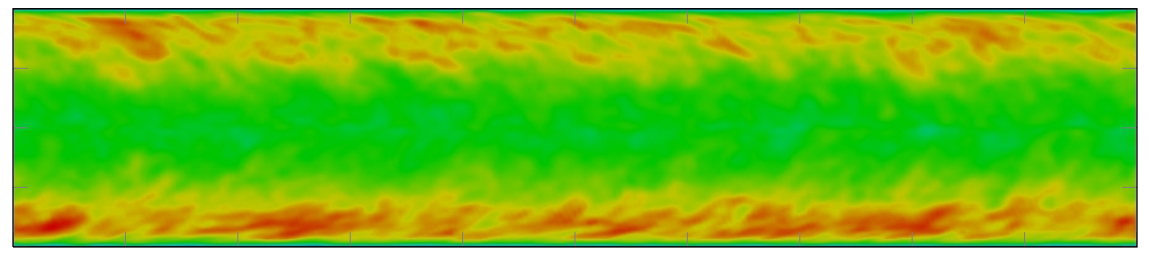

(a) RMS v velocity, time average, constant mass flux, no control

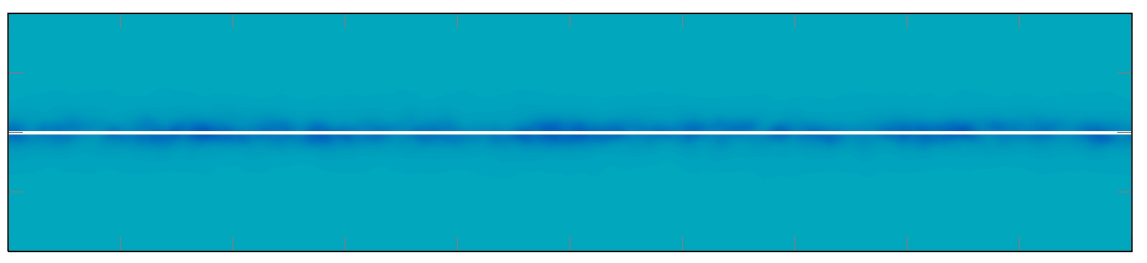

(b) RMS v velocity, time average, constant mass flux, oscillatory wall, $\mathrm{A}=0.5, \Omega=$ 0.35

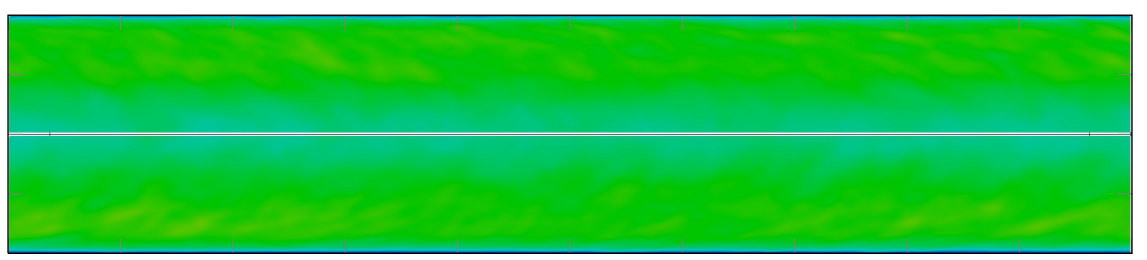

(c) RMS v velocity, time average, constant mass flux, phase randomization with $T^{+}=$ 875

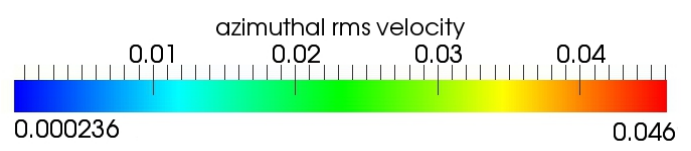

Figure 5.18: RMS azimuthal velocity, time average, constant mass flux, controlled and uncontrolled cases. 


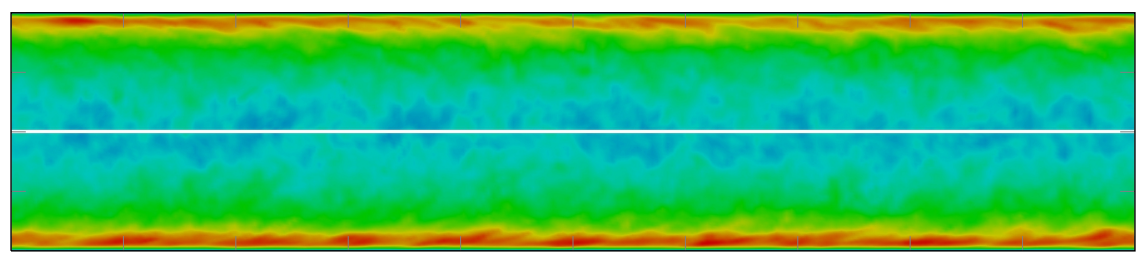

(a) RMS r-vorticity, time average, constant mass flux, no control

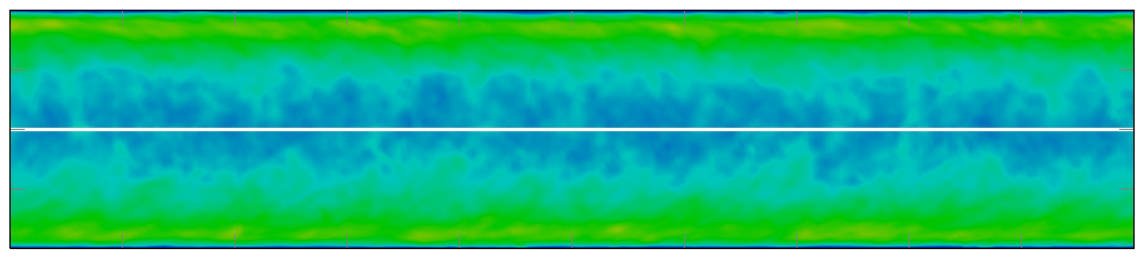

(b) RMS r-vorticity, time average, constant mass flux, wall oscillations

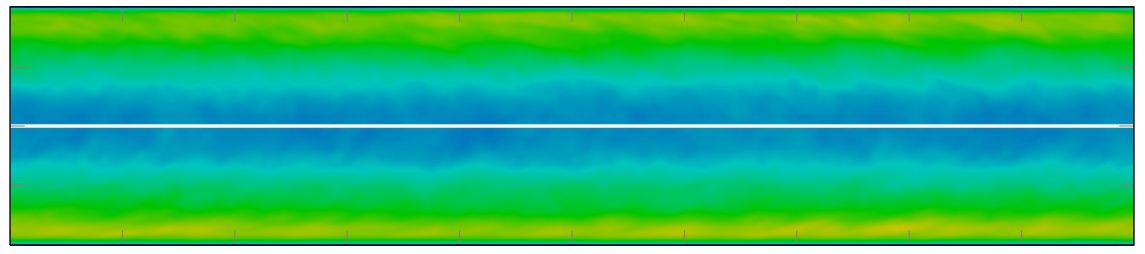

(c) RMS r-vorticity, time average, constant mass flux, phase randomization

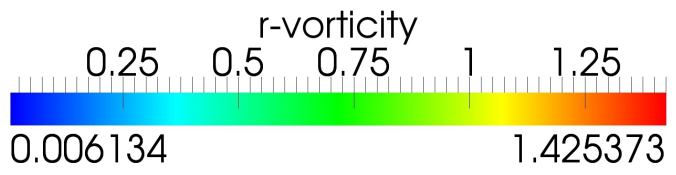

Figure 5.19: RMS r-vorticity, time average, constant mass flux, controlled and uncontrolled cases. 


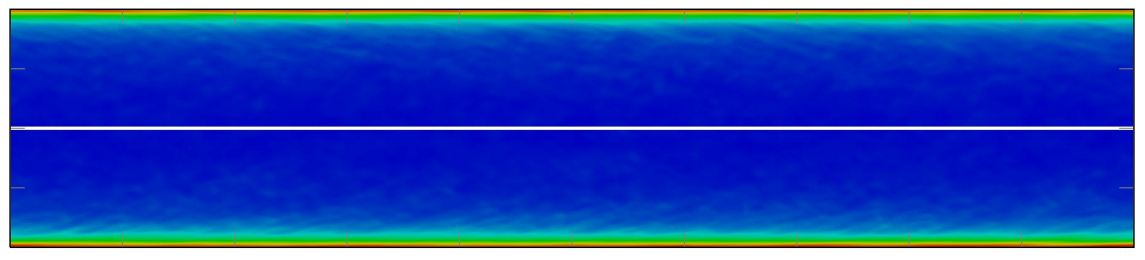

(a) RMS $\theta$-vorticity, time average, constant mass flux, no control

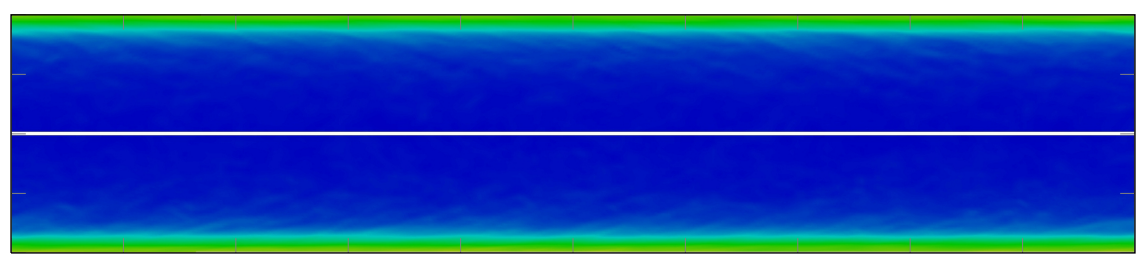

(b) RMS $\theta$-vorticity,time average, constant mass flux, wall oscillation

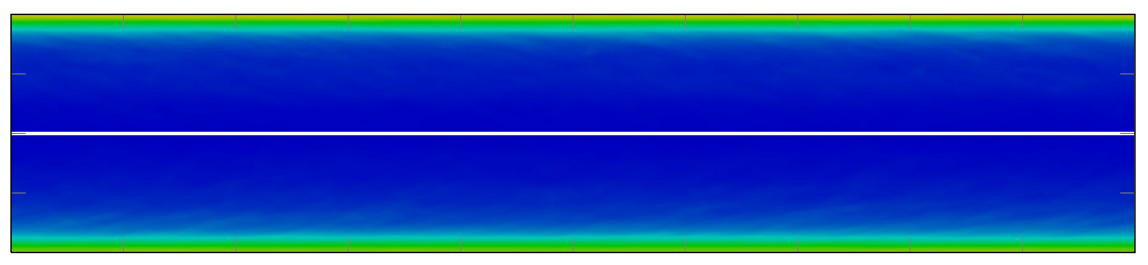

(c) RMS $\theta$-vorticity,time average, constant mass flux, phase randomization

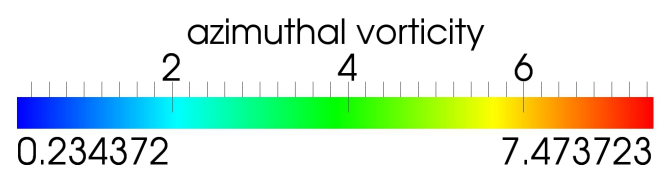

Figure 5.20: RMS $\theta$-vorticity, time average, constant mass flux controlled and uncontrolled cases. 


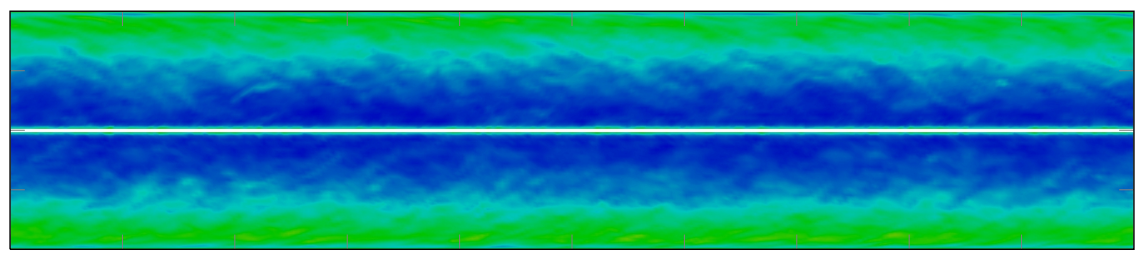

(a) RMS z-vorticity,time average, constant mass flux, no control

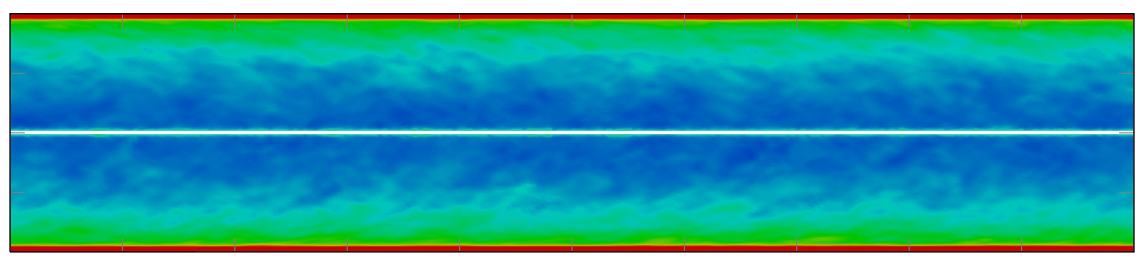

(b) RMS z-vorticity,time average, constant mass flux, wall oscillation

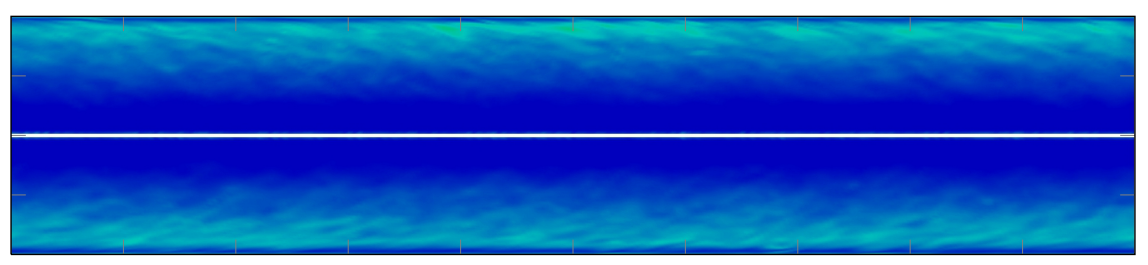

(c) RMS z-vorticity,time average, constant mass flux, phase randomization

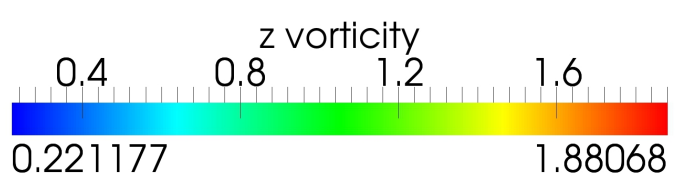

Figure 5.21: RMS z-vorticity,time average, constant mass flux, controlled and uncontrolled cases. 


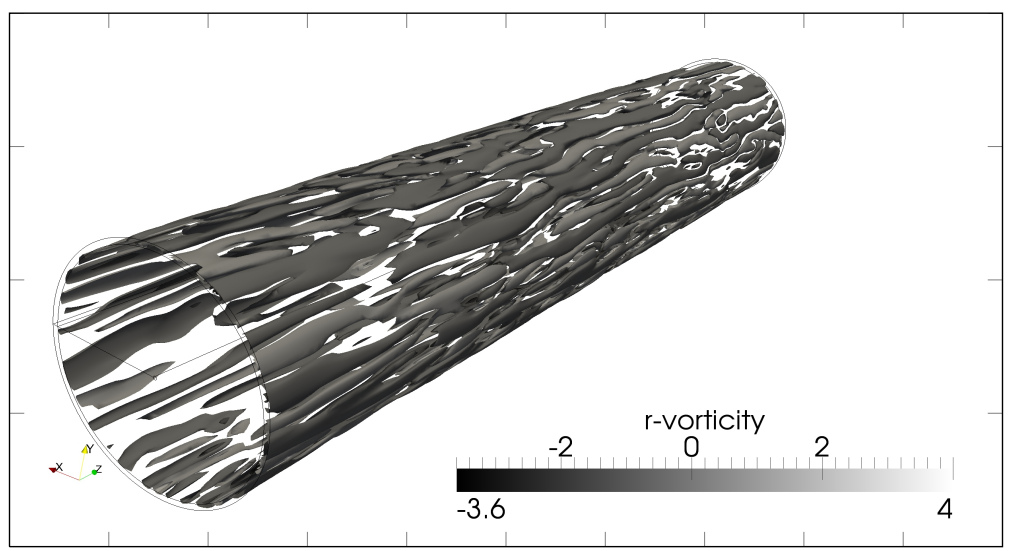

(a) Average rms r vorticity, uncontrolled case, wall region, constant mass flux

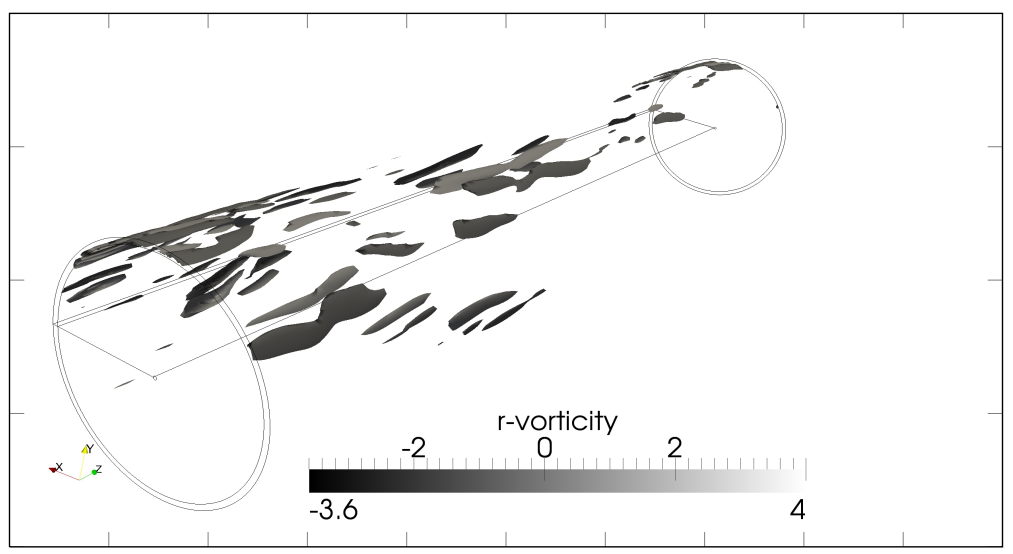

(b) Average rms r vorticity wall oscillations, wall region, constant mass flux

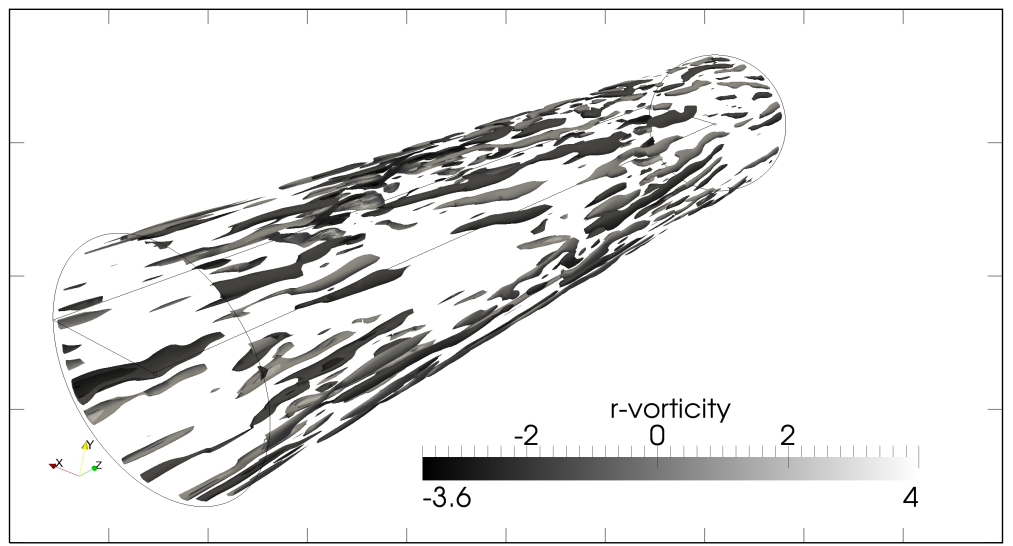

(c) Average rms r vorticity phase randomization, wall region, constant mass flux

Figure 5.22: Average rms r vorticity, wall region, constant mass flux, controlled and uncontrolled cases 


\section{CHAPTER 6}

\section{CONCLUSION}

There is no conversation more boring than the one where everybody agrees.

Michel Eyquem de Montaigne

Employing solenoidal basis functions for the numerical study of incompressible fluid flow has some benefits. The main benefit is that the velocity field obtained is strictly solenoidal (i.e. divergence-free) throughout the solution domain for all time. The exact satisfaction of the continuity equation due to the solenoidal nature of the employed bases eliminates the possible detrimental effects of the errors associated with the handling of incompressibilty condition, which is known to be a common problem for incompressible fluid flow [58. Furthermore, as the pressure variable is eliminated via the projection onto the dual solenoidal basis functions, the resulting computer code is simplified, and a separate pressure solver is not necessary hence the problems associated with boundary conditions for pressure, which can be quite problematic [57, 58, 59], are avoided.

Further the obtained solution is much more likely to correspond to physical solution as it is free of simplifications and assumptions found in other popular methods with respect to the pressure boundary conditions and the treatment of the incompressibility condition [59]. In contrast to the popular solenoidal bases obtained from KarhunenLoeve (KL) analysis (also known as Principal Orthogonal Decomposition), no separate simulation stage is required in the generation of the current bases. KL bases are known to be optimal in the energy sense. On the other hand, they are no longer optimal when used for off-reference parameter values, while the current bases are parameter- 
independent.

It is also shown that, the method is effective in calculating the turbulence statistics at moderate Reynolds numbers, and has excellent energy conserving properties. In addition to other advantages, with the use of solenoidal basis functions, constant mass flux formulation is relatively easy. The usage of the analytic solenoidal basis functions for the solution of incompressible flow problems has some disadvantages, arguably the most important of these disadvantages is the limitation to simple geometries in the current formulation. However, there are domain decomposition approaches (see [34]) as a possible remedy. However, the reader should note that, direct numerical simulations (DNS), themselves are computationally expensive, and hence are limited to simple geometries, therefore the use of solenoidal bases does not present a significant disadvantage in terms of electable geometries.

In this study the solenoidal basis functions were utilized in constructing a spectral method with which the unsteady incompressible Navier-Stokes equations were solved numerically for the case of pipe flow. For the simulation both constant pressure gradient and constant mass flux cases were treated. The code has been validated for the linear stability results as well as turbulence statistics at moderate Reynolds numbers. The method was also shown to posses excellent energy conserving properties. Following favorable validation results, effects of two drag reduction mechanisms were also simulated. These mechanisms are spanwise wall oscillations and phase randomization. Effects of phase randomization for pipe flow were investigated for the first time in literature, in addition the effects of phase randomization coupled with constant flow rate, too, were treated for the first time. Both drag reduction mechanisms were shown to work at different extents, and the case of drag reduction via wall oscillation was validated using results found in the literature.

It is observed that both wall oscillations and phase randomization cause a decrease in vorticity, root mean square cross-velocity (azimuthal and radial) magnitudes and diminish vortical activity near the wall region, in accordance with earlier works in drag reduction [38, 42, 93]. The simulations show that phase randomization is more effective and abrupt in flow-relaminarization when compared to wall oscillations. Differences in the nature of drag reduction for phase randomization and wall oscillations were iden- 
tified during the course of this study, with phase randomization being more effective in suppressing radial cross-flow component and the azimuthal vorticity component, whereas wall-oscillations being relatively better at diminishing azimuthal cross-flow component and radial vorticity component.

Spanwise wall oscillations can be controlled by varying the oscillation amplitude and the frequency, both in real life applications and numerical simulations. In the case of phase randomization, the parameters to be considered are the selection of the modes whose phases will be randomized and the randomization period. This renders, combined with the comparatively long lasting transients, parametric studies using phase randomization relatively difficult. It must be stated that, the real world application of phase randomization is less obvious, as stated previously, specially arranged arrays of finite wall protrusions were suggested as a candidate for the physical counterpart of phase randomization [45], as were addition of polymer chains [44]. One other important point in comparing the drag reduction mechanisms involved is that, phase randomization does not significantly alter the mixing properties of the underlying flow (as an example consult Figs. 5.16(b), 5.16(c)], whereas spanwise wall oscillations would conceivably enhance mixing while diminishing turbulent activity.

\subsection{Future Work}

The work presented here can be extended or enhanced in a number of ways. Some of these possible enhancements are listed below,

- The alleged link between polymer addition and phase randomization can be investigated. This would either involve a different code for the simulation of the polymer additives or using the outlook in [94] a many body solver can be coupled with the present numerical code. If such a link indeed exists, modelling via phase randomization can result in faster simulations for certain cases of particulate flow.

- The possible production of stabilizing traveling waves due to wall oscillations and phase randomization in a lower Reynolds number range $(\operatorname{Re} \sim 3000)$ could be investigated. 
- Effects of phase randomization and spanwise wall oscillations on turbulent lifetimes could be investigated, using [95] as a reference to build upon.

- Larger Reynolds numbers can be investigated to test the effectiveness of the numerical approach at flow parameters close to some real life applications, although this would require parallelization of the code on a larger number of processing cores (currently 4 are used via OpenMp), with the possible use of graphics processing units.

- Connected to the above, the code can be (re)parallelized using MPI. 


\section{REFERENCES}

[1] H. Tennekes and J. Lumley, A first course in turbulence. Mit Press, 1972.

[2] J. O. Hinze, Turbulence. McGraw-Hill classic textbook reissue series. - New York : McGraw-Hill, New York: McGraw-Hill, 1987.

[3] P. Holmes, J. L. Lumley, and G. Berkooz, Turbulence, coherent structures, dynamical systems, and symmetry. Cambridge University Press, 1998.

[4] U. Frisch, Turbulence. The legacy of A. N. Kolmogorov. 1995.

[5] S. B. Pope, Turbulent flows. Sept. 2000.

[6] T. Bohr, M. H. Jensen, G. Paladin, and A. Vulpiani, Dynamical systems approach to turbulence. Cambridge University Press, Aug. 1998.

[7] P. Cvitanović, R. Artuso, R. Mainieri, G. Tanner, G. Vattay, and A, "Chaos : classical and quantum," vol. I, 2009.

[8] P. Holmes, J. L. Lumley, and G. Berkooz, Turbulence, coherent structures, dynamical systems and symmetry. Cambridge: Cambridge University Press, 1996.

[9] A. Talamelli, F. Persiani, F. HM, and Others, "CICLoPE - a response to the need for high Reynolds number experiments," Fluid dynamics research, vol. 41, p. 021407, 2009.

[10] J. M. L. Poiseuille, "Recherches experimentales sur ie mouvement des liquides dans les tubes de tres petits diametres," C. R. Acad. Sci., vol. 11, 1840.

[11] G. Hagen, Handbuch der wasserbaukunst: Th., 1.-4. bd. die ströme: uferschaälungen, strombauten und schiffahrts-canäle. 1871-74. Handbuch der Wasserbaukunst, Ernst \& Korn, 1874.

[12] O. Reynolds, "An experimental investigation of the circumstances which determine whether the motion of water shall be direct or sinuous, and of the law of resistance in parallel channels," Philosophical Transactions of the Royal Society of London, vol. 174, no. 1883, pp. 935-982, 1883.

[13] L. N. Meseguer, A.; Trefethen, "Linearized pipe flow to Reynolds number 10^7," Journal of Computational Physics, vol. 186, pp. 178-197, Mar. 2003.

[14] B. Eckhardt, T. M. Schneider, B. Hof, and J. Westerweel, "Turbulence transition in pipe flow," Annual Review of Fluid Mechanics, vol. 39, pp. 447-468, Jan. 2007.

[15] R. R. Kerswell, "Recent progress in understanding the transition to turbulence in a pipe," Nonlinearity, vol. 18, pp. R17-R44, Nov. 2005. 
[16] T. Mullin and J. Peixinho, "Transition to turbulence in pipe flow," Journal of Low Temperature Physics, vol. 145, pp. 75-88, Nov. 2006.

[17] J. Peixinho and T. Mullin, "Decay of turbulence in pipe flow," Physical Review Letters, vol. 96, pp. 1-4, Mar. 2006.

[18] B. Hof, C. W. H. van Doorne, J. Westerweel, F. T. M. Nieuwstadt, H. Faisst, B. Eckhardt, H. Wedin, R. R. Kerswell, and F. Waleffe, "Experimental observation of nonlinear traveling waves in turbulent pipe flow.," Science (New York, N.Y.), vol. 305, pp. 1594-8, Sept. 2004.

[19] P. Manneville, Instabilities, chaos and turbulence: an introduction to nonlinear dynamics and complex systems. Imperial College Press, 2004.

[20] P. Cvitanović, "Invariant measurement of strange sets in terms of cycles," Physical Review Letters, vol. 61, no. 24, 1988.

[21] R. Artuso, E. Aurell, and P. Cvitanovic, "Recycling of strange sets: i. cycle expansions," Nonlinearity, vol. 325, 1990.

[22] M. Nagata, "Three-dimensional finite-amplitude solutions in plane Couette flow: bifurcation from infinity," Journal of Fluid Mechanics, vol. 217, pp. 519-527, 1990 .

[23] F. Waleffe, "Three-dimensional coherent states in plane shear flows," Physical Review Letters, vol. 81, pp. 4140-4143, Nov. 1998.

[24] F. Waleffe, "Exact coherent structures in channel flow," Journal of Fluid Mechanics, vol. 435, pp. 93-102, June 2001.

[25] F. Waleffe, "Homotopy of exact coherent structures in plane shear flows," Physics of Fluids, vol. 15, no. 6, p. 1517, 2003.

[26] J. F. Gibson, J. Halcrow, and P. Cvitanović, "Visualizing the geometry of state space in plane Couette flow," Journal of Fluid Mechanics, vol. 611, pp. 107-130, Aug. 2008.

[27] P. Moin and K. Mahesh, "Direct numerical simulation: a tool in turbulence research," Annual Review of Fluid Mechanics, vol. 30, pp. 539-578, Jan. 1998.

[28] T. Schneider, B. Eckhardt, and J. Vollmer, "Statistical analysis of coherent structures in transitional pipe flow," Physical Review E, vol. 75, June 2007.

[29] A. Duggleby, K. Ball, M. Paul, and P. Fischer, "Dynamical eigenfunction decomposition of turbulent pipe flow," Journal of Turbulence, vol. 8, no. 772815469, 2007.

[30] J. G. M. Eggels, F. Unger, M. H. Weiss, J. Westerweel, R. J. Adrian, R. Friedrich, and F. T. M. Nieuwstadt, "Fully developed turbulent pipe flow: a comparison between direct numerical simulation and experiment," Journal of Fluid Mechanics, vol. 268, no. -1, pp. 175-210, 1994.

[31] K. Fukagata, "Highly energy-conservative finite difference method for the cylindrical coordinate system," Journal of Computational Physics, vol. 181, pp. 478498, Sept. 2002. 
[32] R. Moser, P. Moin, and a. Leonard, "A spectral numerical method for the NavierStokes equations with applications to Taylor-Couette flow," Journal of Computational Physics, vol. 52, pp. 524-544, Dec. 1983.

[33] A. Leonard and A. Wray, "A new numerical method for the simulation of threedimensional flow in a pipe," Tech. Rep. 19820023768, NASA, 1982.

[34] F. Pasquarelli, "Domain decomposition for spectral approximation to Stokes equations via divergence free functions," Applied Numerical Mathematics, pp. 493-514, 1991.

[35] F. Pasquarelli, A. Quarteroni, and G. Sacchi-Landriani, "Spectral approximations of the Stokes problem by divergence-free functions," Journal of scientific computing, vol. 2, no. 3, pp. 195-226, 1987.

[36] N. M. G. Mhuiris, "The construction and use of divergence free vector expansions for incompressible fluid flow calculations," Tech. Rep. 86, NASA, 1986.

[37] W. J. Jung, N. Mangiavacchi, and F. L. Akhavan, "Suppression of turbulence in wall-bounded flows by high-frequency spanwise oscillations," Physics of Fluids A, vol. 4, no. 8, pp. 1605-1608, 1992.

[38] K.-S. Choi and M. Graham, "Drag reduction of turbulent pipe flows by circularwall oscillation," Physics of Fluids, vol. 10, no. 1, p. 7, 1998.

[39] N. V. Nikitin, "On the mechanism of turbulence suppression by spanwise surface oscillations," Fluid Dynamics, no. 2, pp. 185-190, 2000.

[40] M. Quadrio and S. Sibilla, "Numerical simulation of turbulent flow in a pipe oscillating around its axis," Journal of Fluid Mechanics, vol. 424, pp. 217-241, Dec. 2000.

[41] M. Quadrio and P. Ricco, "Critical assessment of turbulent drag reduction through spanwise wall oscillations," Journal of Fluid Mechanics, vol. 521, pp. 251-271, Dec. 2004.

[42] A. Duggleby, K. S. Ball, and M. R. Paul, "The effect of spanwise wall oscillation on turbulent pipe flow structures resulting in drag reduction," Physics of Fluids, vol. 19, no. 12, p. 125107, 2007.

[43] J. M. J. Den Toonder, M. a. Hulsen, G. D. C. Kuiken, and F. T. M. Nieuwstadt, "Drag reduction by polymer additives in a turbulent pipe flow: numerical and laboratory experiments," Journal of Fluid Mechanics, vol. 337, pp. 193-231, Apr. 1997.

[44] R. A. Handler, E. Levich, and L. Sirovich, "Drag reduction in turbulent channel flow by phase randomization," Physics of Fluids A, vol. 5, no. 3, pp. 686-695, 1993.

[45] L. Sirovich and S. Karlsson, "Turbulent drag reduction by passive mechanisms," Nature, no. February, pp. 728-730, 1997.

[46] M. D. Gunzburger, Perspectives in flow control and optimization. Philadelphia, PA, USA: Society for Industrial and Applied Mathematics, 2002. 
[47] P. Ricco and M. Quadrio, "Wall-oscillation conditions for drag reduction in turbulent channel flow," International Journal of Heat and Fluid Flow, vol. 29, pp. 891-902, Aug. 2008.

[48] M. Quadrio, "Initial response of a turbulent channel flow to spanwise oscillation of the walls," Journal of Turbulence, no. December, pp. 37-41, 2003.

[49] M. R. Dhanak and C. Si, "On reduction of turbulent wall friction through spanwise wall oscillations," Journal of Fluid Mechanics, vol. 383, pp. 175-195, Mar. 1999.

[50] K.-S. Choi, "Near-wall structure of turbulent boundary layer with spanwise-wall oscillation," Physics of Fluids, vol. 14, no. 7, p. 2530, 2002.

[51] K. S. Ball, R. A. Handler, and L. Sirovich, "Propagating structures in wallbounded turbulent flows," in 12th Symposium on Turbulence (X. B. Reed Jr., G. K. Patterson, \& J. L. Zakin, ed.), p. 9, 1990.

[52] J. Xu, M. R. Maxey, and G. E. Karniadakis, "Numerical simulation of turbulent drag reduction using micro-bubbles," Journal of Fluid Mechanics, vol. 468, pp. 271-281, Oct. 2002.

[53] S. L. Ceccio, "Friction drag reduction of external flows with bubble and gas injection," Annual Review of Fluid Mechanics, vol. 42, pp. 183-203, Jan. 2010.

[54] J. Hœ pffner and K. Fukagata, "Pumping or drag reduction?," Journal of Fluid Mechanics, vol. 635, p. 171, Sept. 2009.

[55] K. Fukagata, "Drag reduction by wavy surfaces," Journal of Fluid Science and Technology, vol. 5, no. 1, p. 1, 2010.

[56] B. B. K. Lieu and R. Moarref, "Controlling the onset of turbulence by streamwise traveling waves. Part 2. Direct Numerical Simulations," October, no. 2009, 2010.

[57] P. M. Gresho and R. L. Sani, "On pressure boundary conditions for the incompressible navier-stokes equations," International Journal for Numerical Methods in Fluids, vol. 7, no. 10, pp. 1111-1145, 1987.

[58] P. M. Gresho, "Incompressible fluid dynamics - Some fundamental formulation issues," Annual Review of Fluid Mechanics, vol. 23, pp. 413-453, 1991.

[59] D. Rempfer, "On boundary conditions for incompressible navier-stokes problems," Applied Mechanics Reviews, vol. 59, no. 3, pp. 107-125, 2006.

[60] H. Choi and P. Moin, "Effects of the computational time step on numerical solutions of turbulent flow," J. Comput. Phys., vol. 113, pp. 1-4, July 1994.

[61] A. J. Chorin, "Numerical solution of the navier-stokes equations," Mathematics of Computation, vol. 22, no. 104, pp. pp. 745-762, 1968.

[62] B. Schmitt and W. von Wahl, "Decomposition of solenoidal fields into poloidal fields, toroidal fields and the mean flow. applications to the boussinesqequations," in The Navier-Stokes Equations II - Theory and Numerical Methods (J. Heywood, K. Masuda, R. Rautmann, and V. Solonnikov, eds.), vol. 1530 of Lecture Notes in Mathematics, pp. 291-305, Springer Berlin / Heidelberg, 1992. 10.1007/BFb0090349. 
[63] O and Daube, "Resolution of the $2 \mathrm{~d}$ navier-stokes equations in velocity-vorticity form by means of an influence matrix technique," Journal of Computational Physics, vol. 103, no. 2, pp. $402-414,1992$.

[64] L. S. Tuckerman, "Divergence-free velocity fields in nonperiodic geometries," $J$. Comput. Phys., vol. 80, pp. 403-441, Feb. 1989.

[65] T. Ohwada and P. Asinari, "Artificial compressibility method revisited: Asymptotic numerical method for incompressible Navier-Stokes equations," Journal of Computational Physics, vol. 229, pp. 1698-1723, Mar. 2010.

[66] J. C. Heinrich, O. C. Zienkiewicz, and R. S. Marshall, "Penalty function solution of coupled convective and conductive heat transfer," in Numerical Methods in Laminar and Turbulent Flow (C. Taylor, K. Morgan, \& C. A. Brebbia, ed.), pp. 935-946, 1978.

[67] F. H. Harlow and J. E. Welch, "Numerical calculation of time-dependent viscous incompressible flow of fluid with free surface," Physics of Fluids, vol. 8, no. 12, pp. 2182-2189, 1965.

[68] S. Le Borne and D. Cook, "Construction of a discrete divergence-free basis through orthogonal factorization in H-arithmetic," Computing, vol. 81, pp. 215238, Nov. 2007.

[69] L. N. Trefethen, "Fourth-order time-stepping for stiff pdes," Society, vol. 26, no. 4, pp. 1214-1233, 2005.

[70] F. Mellibovsky and A. Meseguer, "Pipe flow transition threshold following localized impulsive perturbations," Physics of Fluids, vol. 19, no. 4, p. 044102, 2007.

[71] F. Mellibovsky and A. Meseguer, "Critical threshold in pipe flow transition.," Philosophical transactions. Series A, Mathematical, physical, and engineering sciences, vol. 367, pp. 545-60, Feb. 2009.

[72] F. Mellibovsky and B. Eckhardt, "Takens-Bogdanov bifurcation of travellingwave solutions in pipe flow," Journal of Fluid Mechanics, pp. 1-34, Jan. 2011.

[73] F. Mellibovsky, A. Meseguer, T. M. Schneider, and B. Eckhardt, "Transition in localized pipe flow turbulence," Physical Review Letters, vol. 054502, no. July, pp. 1-4, 2009.

[74] O. Tugluk and H. I. Tarman, "Direct numerical simulation of pipe flow using a solenoidal spectral method," Acta Mechanica, pp. 1-13, 2012. 10.1007/s00707011-0602-z.

[75] E. Buckingham, "On physically similar systems," Physical Review D, vol. 4, no. 4, pp. 345-376, 1914.

[76] K. Fukagata, "Theoretical studies on friction drag reduction control with the aid of direct numerical simulation-a review," Integration The Vlsi Journal, vol. 13, no. 4, pp. 96-106, 2008. 
[77] L. N. Meseguer, A.; Trefethen, "A spectral Petrov-Galerkin formulation for pipe flow: II nonlinear transitional stages," tech. rep., Oxford University Computing Laboratory, Oxford, 2001.

[78] O. Tugluk and H. I. Tarman, "Solenoidal bases for numerical studies of transition in pipe flow," Physica Scripta, vol. T142, p. 014009, Dec. 2010.

[79] O. Tugluk and H. I. Tarman, "Direct numerical simulation of pipe flow using a solenoidal spectral method," Acta Mechanica, vol. 223, pp. 921-935, May 2012.

[80] L. N. Trefethen, Spectral methods in matlab. Philadelphia: Society for Industrial and Applied Mathematics, 2000.

[81] V. Priymak, "Accurate Navier-Stokes investigation of transitional and turbulent flows in a circular pipe," Journal of Computational Physics, vol. 142, pp. 370-411, May 1998.

[82] A. Meseguer and L. N. Trefethen, "A spectral Petrov-Galerkin formulation for pipe flow I: Linear Stability and transient growth," tech. rep., Oxford University Computing Laboratory.

[83] J. Hesthaven, S. Gottlieb, and D. Gottlieb, Spectral methods for time-dependent problems. Cambridge, 2007.

[84] U. Ascher, S. J. Ruuth, and B. T. R. Wetton, "Implicit-explicit methods for time-dependent pde's," SIAM J. Numer. Anal., vol. 32, 1995.

[85] M. Frigo and S. G. Johnson, "The design and implementation of FFTW3," Proceedings of the IEEE, vol. 93, no. 2, pp. 216-231, 2005. Special issue on "Program Generation, Optimization, and Platform Adaptation".

[86] E. Anderson, Z. Bai, C. Bischof, S. Blackford, J. Demmel, J. Dongarra, J. Du Croz, A. Greenbaum, S. Hammarling, A. McKenney, and D. Sorensen, LAPACK users' guide. Philadelphia, PA: Society for Industrial and Applied Mathematics, third ed., 1999.

[87] O. Y. Zikanov, "On the instability of pipe Poiseuille flow," Physics of Fluids, vol. 8, no. 11, p. 2923, 1996.

[88] D. Viswanath and P. Cvitanović, "Stable manifolds and the transition to turbulence in pipe flow," Journal of Fluid Mechanics, vol. 627, p. 215, May 2009.

[89] C. Pringle, A. Willis, and R. Kerswell, "Minimal seeds for shear flow turbulence: using nonlinear transient growth to touch the edge of chaos," Arxiv preprint arXiv:1109.2459, pp. 1-27, 2011.

[90] J. P. Boyd, Chebyshev and Fourier Spectral Methods. DOVER Publications, Inc., second edi ed., 2000.

[91] D. A. Kopriva, Implementing spectral methods for partial differential equations. Springer, 2009.

[92] P. Constantin, M. Cannone, and T. Miyakawa, Mathematical foundation of turbulent viscous flows: lectures given at the C.I.M.E. summer school held in Martina Franca, Italy, September 1-5, 2003. Lecture notes in mathematics, Springer, 2006. 
[93] K.-S. Choi, "European drag-reduction research - recent developments and current status," Fluid Dynamics Research, vol. 26, pp. 325-335, May 2000.

[94] L. H. Zhao, H. I. Andersson, and J. J. J. Gillissen, "Turbulence modulation and drag reduction by spherical particles," Physics of Fluids, vol. 22, no. 8, p. 081702, 2010 .

[95] B. Hof, J. Westerweel, T. M. Schneider, and B. Eckhardt, "Finite lifetime of turbulence in shear flows.," Nature, vol. 443, pp. 59-62, Sept. 2006.

[96] I. S. Gradshteyn and I. M. Ryzhik, Table of Integrals, Series, and Products. Academic Press, 7 ed., Mar. 2007. 


\section{APPENDIX A}

\section{Solenoidal Basis Functions}

We list the forms of the solenoidal basis functions employed in this study below. In all the cases $P_{2 m}$ denotes the Legendre polynomial of order $2 \mathrm{~m}, D_{+}=D+1 / r$, and $D=\frac{d}{d r}$ is replaced by a suitable Legendre differentiation submatrix after discretization based on the Gauss-Legendre grid in the radial direction [80, [78].

Case $\mathbf{I}, \mathrm{l} \neq 0 \mathrm{n}=0$ :

Physical "1-basis":

Dual "1-basis":

$$
\begin{aligned}
& \mathbf{v}^{(1)}=\left(\begin{array}{c}
0 \\
r H \\
0
\end{array}\right) \\
& \tilde{\mathbf{v}}^{(1)}=\left(\begin{array}{c}
0 \\
H \\
0
\end{array}\right) \\
& H=\left(1-r^{2}\right) P_{2 m} \\
& H=P_{2 m}
\end{aligned}
$$

Physical "2-basis":

Dual "2-basis":

$$
\begin{gathered}
\mathbf{v}^{(1)}=\left(\begin{array}{c}
-i l r G \\
0 \\
D_{+}(r G)
\end{array}\right) \\
G=\left(1-r^{2}\right)^{2} P_{2 m}
\end{gathered}
$$

$$
\begin{gathered}
\tilde{\mathbf{v}}^{(1)}=\left(\begin{array}{c}
-i l G \\
0 \\
D_{+} G
\end{array}\right) \\
G=\left(1-r^{2}\right) P_{2 m}
\end{gathered}
$$

Case II, $\mathrm{l}=0 \mathrm{n}=0$ : 
Physical "1-basis":

$$
\begin{gathered}
\mathbf{v}^{(1)}=\left(\begin{array}{c}
0 \\
r H \\
0
\end{array}\right) \\
H=\left(1-r^{2}\right) P_{2 m}
\end{gathered}
$$

Physical "2-basis":

$$
\begin{gathered}
\mathbf{v}^{(2)}=\left(\begin{array}{c}
0 \\
0 \\
H
\end{array}\right) \\
H=\left(1-r^{2}\right) P_{2 m}
\end{gathered}
$$

Case III, $l \neq 0 \quad n \neq 0$ :

Physical "1-basis":

$$
\begin{aligned}
\mathbf{v}^{(1)} & =\left(\begin{array}{c}
-i n r^{(\alpha-1)} G \\
D\left(r^{\alpha} G\right) \\
0
\end{array}\right) \\
G & =\left(1-r^{2}\right)^{2} P_{2 m} \\
\alpha & =\min (|n|, 2-\bmod (n, 2))
\end{aligned}
$$

Physical "2-basis":

$$
\begin{aligned}
\mathbf{v}^{(2)} & =\left(\begin{array}{c}
0 \\
-i l r^{(\alpha+1)} H \\
i n r^{\alpha} H
\end{array}\right) \\
H & =\left(1-r^{2}\right) P_{2 m} \\
\alpha & =\min (|n|, 2-\bmod (n, 2))
\end{aligned}
$$

Dual "1-basis":

$$
\begin{gathered}
\tilde{\mathbf{v}}^{(1)}=\left(\begin{array}{c}
0 \\
H \\
0
\end{array}\right) \\
H=P_{2 m}
\end{gathered}
$$

Dual "2-basis":

$$
\begin{gathered}
\tilde{\mathbf{v}}^{(1)}=\left(\begin{array}{c}
0 \\
0 \\
r H
\end{array}\right) \\
H=P_{2 m}
\end{gathered}
$$

Dual "1-basis":

$$
\begin{aligned}
\tilde{\mathbf{v}}^{(1)} & =\left(\begin{array}{c}
-i n r^{(\alpha-1)} G \\
D\left(r^{\alpha} G\right) \\
0
\end{array}\right) \\
G & =\left(1-r^{2}\right) P_{2 m} \\
\alpha & =\bmod (n, 2)+1
\end{aligned}
$$

Dual "2-basis":

$$
\begin{aligned}
\tilde{\mathbf{v}}^{(1)} & =\left(\begin{array}{c}
0 \\
-i l r^{\alpha} H \\
i n r^{(\alpha-1)} H
\end{array}\right) \\
H & =P_{2 m} \\
\alpha & =2-\bmod (n, 2)
\end{aligned}
$$




\section{APPENDIX B}

\section{Constant Mass Flux Formulation}

While using a constant pressure gradient to drive the flow, the numerical manifestation of this forcing is trivial. In the case constant mass flux however, this is not the case. Some prior work is necessary to ensure the mass flux is indeed kept constant.

First let us consider the open form of the NS equations in cylindrical coordinates, i.e Equation (2.5),

$$
\begin{aligned}
u_{t}+u u_{r}+\frac{1}{r} v u_{\theta}+w u_{z}-\frac{1}{r} v^{2} & =-p_{r}+\frac{1}{R e}\left[u_{r r}+\frac{1}{r} u_{r}+\frac{1}{r^{2}} u_{\theta \theta}+u_{z z}-\frac{2}{r^{2}} v_{\theta}-\frac{1}{r^{2}} u\right] \\
v_{t}+u v_{r}+\frac{1}{r} v v_{\theta}+w v_{z}+\frac{1}{r} u v & =-\frac{1}{r} p_{\theta}+\frac{1}{R e}\left[v_{r r}+\frac{1}{r} v_{r}+\frac{1}{r^{2}} v_{\theta \theta}+v_{z z}+\frac{2}{r^{2}} u_{\theta}-\frac{1}{r^{2}} v\right] \\
w_{t}+u w_{r}+\frac{1}{r} v w_{\theta}+w w_{z} & =-p_{z}+\frac{1}{R e}\left[w_{r r}+\frac{1}{r} w_{r}+\frac{1}{r^{2}} w_{\theta \theta}+w_{z z}\right]
\end{aligned}
$$

together with the continuity equation,

$$
u_{r}+\frac{1}{r} v_{\theta}+w_{z}+\frac{1}{r} u=0
$$

The objective is to find the pressure gradient, G that enforces constant mass flux $Q$ along the pipe where,

$$
Q=\int_{0}^{2 \pi} \int_{0}^{1} w r d r d \theta
$$

Note that by incompressibility, 


$$
\begin{aligned}
\frac{\partial Q}{\partial z} & =\int_{o}^{2 \pi} \int_{0}^{1} \frac{\partial w}{\partial z} r d r d \theta \\
& =-\int_{o}^{2 \pi} \int_{0}^{1}\left(u_{r}+\frac{1}{r} v_{\theta}+\frac{1}{r} u\right) r d r d \theta \\
& =-\int_{o}^{2 \pi} \int_{0}^{1}\left(\frac{\partial}{\partial r}(r u)-\not x+v_{\theta}+\not x\right) d r d \theta \\
& =-\int_{0}^{2 \pi} \underbrace{\left.[r u]\right|_{r=0} ^{1}}_{0 \text { by no-slip b.c. }} d \theta-\int_{0}^{1} \underbrace{\left.[v]\right|_{\theta=0} ^{2 \pi}}_{0 \text { by periodicity }} d r=0 \\
\Rightarrow Q=Q(t) \quad \text { only. } &
\end{aligned}
$$

In order to determine the pressure gradient $\mathrm{G}$ that generates constant $\mathrm{Q}$, we need to relate $\mathrm{G}$ to $\mathrm{Q}$. Let us start with the definition of $\mathrm{G}$, which is the mean pressure gradient in the z-direction:

$$
\begin{aligned}
G & =\frac{1}{\pi L} \int_{0}^{L} \int_{o}^{2 \pi} \int_{0}^{1}\left[-\frac{\partial p}{\partial z}\right] r d r d \theta d z . \\
& =\frac{1}{\pi L} \int_{0}^{L} \int_{o}^{2 \pi} \int_{0}^{1}\left[w_{t}+u w_{r}+\frac{1}{r} v w_{\theta}+w w_{z}\right. \\
& \left.-\frac{1}{R e}\left[w_{r r}+\frac{1}{r} w_{r}+\frac{1}{r^{2}} w_{\theta \theta}+w_{z z}\right]\right] r d r d \theta d z
\end{aligned}
$$

After some manipulation, the above relation leads to,

$$
G=\frac{1}{\pi L}\left\{\frac{\partial}{\partial t} \int_{0}^{L} Q d z-\left.\frac{1}{R e} \int_{0}^{L} \int_{0}^{2 \pi} \frac{\partial w}{\partial r}\right|_{r=1} d \theta d z\right\} .
$$

If one wants to keep the flux Q constant, i.e. time independent, then,

$$
G=-\left.\frac{1}{\pi L R e} \int_{0}^{L} \int_{0}^{2 \pi} \frac{\partial w}{\partial r}\right|_{r=1} d \theta d z
$$

Considering the Fourier representation of velocity,

$$
w=\hat{w}(r) e^{i(n \theta+2 \pi l z / L)},
$$

results in,

$$
G=-\frac{2}{R e} \frac{\partial \hat{w}}{\partial r}(l=0, n=0, r=1) .
$$

Using the solenoidal representation, 


$$
\hat{w}(l=0, n=0, r)=\sum_{m} a_{m}^{2}(l=0, n=0, t)\left(1-r^{2}\right) P_{2 m},
$$

where $P_{2 m}$ is the legendre polynomial of order $2 \mathrm{~m}$, results in,

$$
G=\frac{4}{R e} \sum_{m} a_{m}^{2}(l=0, n=0, t)
$$

Furthermore, one can obtain the constraining equation for the expansion coefficients for a given flux $\mathrm{Q}$,

$$
\begin{aligned}
Q & =\int_{o}^{2 \pi} \int_{0}^{1} w r d r d \theta \\
& =2 \pi \int_{0}^{1} \hat{w}(l=0, n=0, r) r d r
\end{aligned}
$$

where $l=0$ follows from $Q=Q(t)$ only, and finally

$$
Q=2 \pi \sum_{m} a_{m}^{2}(l=0, n=0, t) \underbrace{\int_{0}^{1} r\left(1-r^{2}\right) P_{2 m}(r) d r}_{I_{2 m}}
$$

where $I_{2 m}$ is given by [96],

$$
I_{2 m}=\frac{(-1)^{m+1} \Gamma(m+1 / 2)}{8 \sqrt{\pi} \Gamma(m+3)}\left\{\frac{2(m+2)(m-3 / 2)+3}{(m-1 / 2)(m-3 / 2)}\right\},
$$

where $\Gamma$ is the gamma function. Hence, both the required pressure gradient $G$ and the constraining equation (B.2) are now known, and it is ensured that the mass flux is kept constant throughout the simulation by their incorporation into the computer code. 


\section{CURRICULUM VITAE}

\section{Personal \& Contact Information}

Name: Ozan

Surname: Tuğluk

Date of birth: May 1979

Citizenship: Republic of Turkey

e-mail address: e112980@metu.edu.tr, ozantugluk@gmail.com

Postal address: Merkez Muhendislik Binasi, Muhendislik Bilimleri Bolumu MM-805 ODTU, 06800 Cankaya Ankara/TURKEY

Telephone: +90 3122104443 (office), +90 3122300830 (home)

\section{Education}

- 2008 - present P.h.D. student

Department of Engineering Sciences, Computational Mechanics Graduate Program, METU ${ }^{1} /$ Ankara.

- 09/2002 - 01/2005 M.S. in Computational Mechanics

Department of Engineering Sciences, Computational Mechanics Graduate Program, METU/Ankara. Thesis title: Dynamics of Wall Bounded Turbulence. Advisor: Hakan I. Tarman.

- 1997 - 2002 B.S. in Aeronautical Engineering

Department of Aerospace Engineering, METU/Ankara.

\section{Employment Information}

1 Middle East Technical University 
- 01/2004 - 08/2007 : METU Computer Centre, METU/Ankara, Last position held: UNIX system administrator. Administration of campus central UNIX/Linux servers (Debian, AIX, Solaris).

- 07-09/2001 : Internship at OYAK-Renault/Bursa, R\&D department.

- 07-09/2000 : Internship at TAI $\bigsqcup^{2} /$ Ankara, Factory internship.

\section{Research Interests}

- Current research topic : Investigation of various turbulence and transition control strategies in pipe flow via direct numerical simulation. Developed a fortran 90 code employing a selonidal spectral method for the simulation.

- Fields of interest : Transition to turbulence, turbulence control, optimal disturbances in turbulent flow, genetic/evolutionary algorithms, nonlinear dynamics, parallel computing, general purpose gpu programming.

- Some relevant graduate courses :

- AE541 Advanced Computational Fluid Dynamics

- AE546 CFD on Unstructured Grids

- ES503 Finite Element Method

- ES504 Numerical Solution of Partial Diff. Equations

- ES702 Pseudospectral Methods

- PHYS721 Simulation of Many-particle Systems

- IAM565 Introduction to Algorithms and Complexity

Skills and Abilities Computer Related

Operating Systems: Debian GNU/Linux (primary OS), Gentoo GNU/Linux, IBM AIX (5.x series), Sun Solaris $(8,9)$.

Programming Languages: Fortran 90/95 (primary language), Fortran 77, python (familiar), C (familiar).

\footnotetext{
2 Tusaş Aerospace Industries
} 
Document preparation: $\mathrm{AT}_{\mathrm{E} X}$.

Other software : Matlab/octave, gnuplot, paraview, mayavi, openfoam(familiar).

Scripting: bash, perl (familiar).

Languages Turkish (native), English (fluent), Spanish (upper intermediate), French (beginner-intermediate).

\section{Publications}

- Tuğluk, O. and Tarman, I. H., Drag Reduction in Pipe Flow via Phase Randomization, Under preparation

- Tuğluk, O. and Tarman, I. H., Direct Numerical Simulation of Pipe Flow Using a Solenoidal Spectral Method, Acta Mechanica, Accepted for publication 12/2011, DOI: $10.1007 / \mathrm{s} 00707-011-0602-\mathrm{z}$

- Tuğluk, O. and Tarman, I. H., Solenoidal Bases for Numerical Studies of Transition in Pipe Flow, Physica Scripta, T142, 2010

- Tuğluk, O. and Tarman, I. H., Dynamics of Wall Bounded Flow in "Mathematical Methods in Engineering", Springer-Verlag, 2007

- Ozan Tuğluk, Dynamics of Wall Bounded Turbulence, M.S. Thesis, METU, Ankara, December 2004. Advisor: Hakan I. Tarman.

\section{Conferences and Summer Schools}

- Tuğluk, O. and Tarman, I. H., Dynamics of Wall Bounded Flow, Mathematical Methods in Engineering International Symposium, Cankaya University AnkaraTurkey, 2006

- Tuğluk, O. and Tarman, I. H., Solenoidal Bases for Numerical Studies of Transition in Pipe Flow, Turbulent Mixing and Beyond Second International Conference and Advanced School, The Abdus Salam International Centre for Theoretical Physics, Trieste, Italy, 2009 
- Participant, The Second European Summer School on Hydrogen Safet, Belfast UK, 2007

- Participant, OPTPDE - Summer School - Challenges in Applied Control and Optimal Design, Bilbao Spain, 2011

- Participant (with short talk), International School on MHD and Fusion Application, Turunc, Turkey, 2011

\section{References}

- Assoc. Prof. Dr. Hakan I. Tarman, METU Engineering Sciences Department, tarman@metu.edu.tr, +90 3122102385

- Additional references can be supplied upon request 